\author{
Article \\ Doi 10.5943/mycosphere/8/5/5 \\ Copyright $(\mathcal{C}$ Guizhou Academy of Agricultural Sciences
}

\title{
The current status of species in Diaporthe
}

\section{Dissanayake AJ ${ }^{1,2}$, Phillips AJL ${ }^{3}$, Hyde KD ${ }^{2}$, Yan JY', Li XH',}

\begin{abstract}
${ }^{1}$ Beijing Key Laboratory of Environmental Friendly Management on Fruit diseases and Pests in North China, Institute of Plant and Environment Protection, Beijing Academy of Agriculture and Forestry Sciences, Beijing 100097, People's Republic of China.

${ }^{2}$ Center of Excellence in Fungal Research, School of Science, Mae Fah Luang University, Chiang Rai 57100, Thailand

${ }^{3}$ University of Lisbon, Faculty of Sciences, Biosystems and Integrative Sciences Institute (BioISI), Campo Grande, 1749-016 Lisbon, Portugal.
\end{abstract}

Dissanayake AJ, Phillips AJL, Hyde KD, Yan JY, Li XH 2017 - The current status of species in Diaporthe. Mycosphere 8(5), 1106-1156, Doi 10.5943/mycosphere/8/5/5

\begin{abstract}
In this paper we give an account of species in the genus Diaporthe. Since morphological characters are inadequate to define species in this genus, DNA sequence data are essential to differentiate them. We therefore focus this paper on the 171 species for which ex-type/exepitype/ex-isotype/ex-neotype isolates and corresponding molecular data are available and these species are listed alphabetically. Sexual or asexual morph are noted under each species, detailed descriptions of type materials, host records and geographic distribution are provided. Available DNA sequence data from ex-type cultures are listed in Table 1. Phylogenetic relationships of the species are given in a multi-locus phylogenetic tree based on combined ITS, tefl- $\alpha, \beta$-tubulin and CAL sequences.
\end{abstract}

Key words - asexual morph- distribution - hosts - sexual morph - type species

\section{Introduction}

Diaporthe (syn. Phomopsis) species are well-known as pathogens, endophytes or saprobes on a range of economicaly important crops, ornamentals and forest trees (Santos \& Phillips 2009, Santos et al. 2011, Udayanga et al. 2011, 2012a,b, 2014a,b, 2015, Gomes et al. 2013, Hyde et al. 2014, Dissanayake et al. 2015, 2017a, b, Fan et al. 2015). Their frequent association with plant diseases has stimulated considerable interest in this genus.

The genus Diaporthe was introduced by Nitschke (1870) with D. eres as the type species. It is placed in the family Diaporthaceae, order Diaporthales, in the class Sordariomycetes (Maharachchikumbura et al. 2015, 2016). For many years species in Diaporthe were introduced largely on the basis of host association, which resulted in a proliferation of species names. However, it is now recognised that species are not host-specific and a single species can be found on more than one host (Rehner \& Uecker 1994). Furthermore, several different species can be found on a single host (Mostert et al. 2001b). Since morphology is of limited value in defining species (Sutton 1980, Rehner \& Uecker 1994, Chi et al. 2007, Hyde et al. 2011) taxonomy of the genus relies largely on molecular phylogenies, especially those derived from sequences of ITS, tefl- $\alpha, \beta$-tubulin and CAL loci (Udayanga et al. 2012a, Gomes et al. 2013). Thus all of the older species names linked to Diaporthe or Phomopsis, and for which cultures or DNA sequence data are not available, cannot be linked to the species in this genus. Such older taxa will have to be disregarded unless they are epitypified. 
Udayanga et al. (2012a) re-evaluated the phylogenetic species recognition in Diaporthe using a multi-locus phylogeny based on a combined data matrix of ITS, and partial sequences from the translation elongation factor $1-\alpha, \beta$ tubulin and calmodulin molecular markers. They included DNA sequences of available 26 ex-type cultures, providing a multi-locus backbone tree. Gomes et al. (2013) provided detailed descriptions of 54 species of Diaporthe that were linked to the types, while Hyde et al. (2014) provided a backbone phylogenetic tree of 72 species, also derived from the types. In this paper we focus on the 171 species that are currently known from culture or from direct sequencing, and are linked to their holotype, epitype, isotype or neotype and that can now be recognised with molecular data. Hence, this work serves as the first comprehensive overview of all species in Diaporthe since 2012.

Table 1 GenBank accession numbers of species treated in the phylogenetic analysis (Fig. 1). Extype/ex-epitype/ex-isotype/ex-neotype isolates are marked with an asterisk mark.

\begin{tabular}{|c|c|c|c|c|c|c|}
\hline Species & Isolate & Host & ITS & $\beta$-tubulin & tef1- $\alpha$ & CAL \\
\hline Diaporthe acaciarum & CBS $138862 *$ & Acacia tortilis & KP004460 & КР004509 & $N / A^{a}$ & $\mathrm{~N} / \mathrm{A}^{\mathrm{a}}$ \\
\hline D. acaciigena & CBS $129521^{*}$ & Acacia retinodes & KC343005 & KC343973 & KC343731 & KC343247 \\
\hline D. acericola & MFLUCC 17-0956* & Acer negundo & KY964224 & KY964074 & KY964180 & KY964137 \\
\hline D. acutispora & CGMCC $3.18285^{*}$ & Coffea sp. & KX986764 & KX999195 & KX999155 & KX999274 \\
\hline D. alleghaniensis & CBS $495.72 *$ & $\begin{array}{l}\text { Betula } \\
\text { alleghaniensis }\end{array}$ & KC343007 & KC343975 & KC343733 & KC343249 \\
\hline \multirow[t]{2}{*}{ D. alnea } & CBS $146.46^{*}$ & Alnus sp. & KC343008 & KC343976 & KC343734 & KC 343250 \\
\hline & CBS 159.47 & Alnus sp. & KC343009 & KC343977 & KC343735 & KC343251 \\
\hline \multirow[t]{2}{*}{ D. ambigua } & CBS $114015^{*}$ & Pyrus communis & KC343010 & KC343978 & KC343736 & KC343252 \\
\hline & CBS 117167 & Aspalathus linearis & KC343011 & KC343979 & KC343737 & KC343253 \\
\hline \multirow[t]{2}{*}{ D. ampelina } & CBS $114016^{*}$ & Vitis vinifera & AF230751 & $\mathrm{JX} 275452$ & AY745056 & AY230751 \\
\hline & CBS 267.80 & Vitis vinifera & KC343018 & KC343986 & KC343744 & KC343260 \\
\hline \multirow[t]{2}{*}{ D. amygdali } & CBS $126679^{*}$ & Prunus dulcis & KC343022 & KC343990 & AY343748 & KC343264 \\
\hline & CBS 111811 & Vitis vinifera & KC343019 & KC343987 & KC343745 & KC343261 \\
\hline D. anacardii & CBS $720.97 *$ & $\begin{array}{l}\text { Anacardium } \\
\text { ocidentale }\end{array}$ & KC343024 & KC343992 & KC343750 & KC343266 \\
\hline \multirow[t]{2}{*}{ D. angelicae } & CBS $111592 *$ & $\begin{array}{l}\text { Heracleum } \\
\text { sphondylium }\end{array}$ & KC343027 & KC343995 & KC343753 & KC343269 \\
\hline & CBS 100871 & Foeniculum vulgare & KC343025 & KC343993 & KC343751 & KC343267 \\
\hline \multirow[t]{2}{*}{ D. apiculatum } & CGMCC $3.17533^{*}$ & Camellia sinensis & KP267896 & KP293476 & KP267970 & N/A ${ }^{a}$ \\
\hline & LC3187 & Camellia sinensis & KP267866 & KP293446 & KP267940 & $N / A^{a}$ \\
\hline \multirow[t]{2}{*}{ D. aquatica } & IFRDCC $3051^{*}$ & Aquatic habitat & JQ797437 & $\mathrm{N} / \mathrm{A}^{\mathrm{a}}$ & $\mathrm{N} / \mathrm{A}^{\mathrm{a}}$ & $\mathrm{N} / \mathrm{A}^{\mathrm{a}}$ \\
\hline & IFRDCC 3015 & Aquatic habitat & JQ797438 & $\mathrm{N} / \mathrm{A}^{\mathrm{a}}$ & N/A ${ }^{a}$ & $\mathrm{~N} / \mathrm{A}^{\mathrm{a}}$ \\
\hline D. $\operatorname{arctii}$ & DP0482* & Arctium lappa & KJ590736 & KJ610891 & KJ590776 & KJ612133 \\
\hline \multirow[t]{2}{*}{ D. arecae } & CBS $161.64^{*}$ & Areca catechu & KC343032 & KC344000 & KC343758 & KC343274 \\
\hline & CBS 535.75 & Citrus sp. & KC343033 & KC344001 & KC343759 & KC343275 \\
\hline D. arengae & CBS $114979 *$ & Arenga engleri & KC343034 & KC344002 & KC343760 & KC343276 \\
\hline D. aseana & MFLUCC 12-0299a* & Unknown dead leaf & KT459414 & KT459432 & KT459448 & KT459464 \\
\hline \multirow[t]{2}{*}{ D. asheicola } & CBS $136967^{*}$ & Vaccinium ashei & KJ160562 & KJ160518 & KJ160594 & KJ160542 \\
\hline & CBS 136968 & Vaccinium ashei & KJ160563 & KJ160519 & KJ160595 & KJ160543 \\
\hline \multirow[t]{2}{*}{ D. aspalathi } & CBS $117169^{*}$ & Aspalathus linearis & KC343036 & KC344004 & KC343762 & KC 343278 \\
\hline & CBS 117168 & Aspalathus linearis & KC343035 & KC344003 & KC343761 & KC343277 \\
\hline D. australafricana & CBS $111886^{*}$ & Vitis vinifera & KC343038 & KC344006 & KC343764 & KC343280 \\
\hline
\end{tabular}




\begin{tabular}{|c|c|c|c|c|c|c|}
\hline & CBS 113487 & Vitis vinifera & KC343039 & KC344007 & KC343765 & KC343281 \\
\hline \multirow[t]{2}{*}{ D. baccae } & CBS 136972* & $\begin{array}{l}\text { Vaccinium } \\
\text { corymbosum }\end{array}$ & KJ160565 & $\mathrm{N} / \mathrm{A}^{\mathrm{a}}$ & KJ160597 & $\mathrm{N} / \mathrm{A}^{\mathrm{a}}$ \\
\hline & CРC 20585 & $\begin{array}{l}\text { Vaccinium } \\
\text { corymbosum }\end{array}$ & KJ160564 & $\mathrm{N} / \mathrm{A}^{\mathrm{a}}$ & KJ160596 & $\mathrm{N} / \mathrm{A}^{\mathrm{a}}$ \\
\hline D. batatas & CBS $122.21 *$ & Ipomoea batatas & KC 343040 & KC344008 & KC343766 & KC343282 \\
\hline D. beilharziae & BRIP 54792* & Indigofera australis & JX862529 & KF170921 & JX862535 & $\mathrm{N} / \mathrm{A}^{\mathrm{a}}$ \\
\hline D. benedicti & BPI 893190* & Salix sp. & KM669929 & $\mathrm{N} / \mathrm{A}^{\mathrm{a}}$ & KM669785 & KM669862 \\
\hline \multirow[t]{2}{*}{ D. betulae } & CFCC 50469* & Betula platyphylla & KT732950 & KT733020 & KT733016 & KT732997 \\
\hline & CFCC 50470 & Betula platyphylla & KT732951 & KT733021 & KT733017 & KT732998 \\
\hline \multirow[t]{2}{*}{ D. betulicola } & CFCC 51128* & Betula albosinensis & KX024653 & KX024657 & KX024655 & KX024659 \\
\hline & CFCC 51129 & Betula albosinensis & KX024654 & KX024658 & KX024656 & KX024660 \\
\hline D. bicincta & CBS 121004* & Juglans sp. & KC343134 & KC344102 & KC343860 & KC343376 \\
\hline \multirow[t]{2}{*}{ D. biconispora } & ZJUD62* & Citrus grandis & KJ490597 & KJ490418 & KJ490476 & KJ490539 \\
\hline & CGMCC 3.17251 & $\begin{array}{l}\text { Fortunella } \\
\text { margarita }\end{array}$ & KJ490596 & KJ490417 & KJ490475 & $\mathrm{N} / \mathrm{A}^{\mathrm{a}}$ \\
\hline \multirow[t]{2}{*}{ D. biguttulata } & ZJUD47* & Citrus limon & KJ490582 & KJ490403 & KJ490461 & $\mathrm{N} / \mathrm{A}^{\mathrm{a}}$ \\
\hline & CGMCC 3.17249 & Citrus limon & KJ490583 & KJ490404 & KJ490462 & $\mathrm{N} / \mathrm{A}^{\mathrm{a}}$ \\
\hline \multirow[t]{2}{*}{ D. biguttusis } & CGMCC $3.17081^{*}$ & Lithocarpus glabra & KF576282 & KF576306 & KF576257 & N/A ${ }^{a}$ \\
\hline & CGMCC 3.17082 & Lithocarpus glabra & KF576283 & KF576307 & KF576258 & $\mathrm{N} / \mathrm{A}^{\mathrm{a}}$ \\
\hline \multirow[t]{2}{*}{ D. brasiliensis } & CBS $133183^{*}$ & $\begin{array}{l}\text { Aspidosperma } \\
\text { tomentosum }\end{array}$ & KC343042 & KC344010 & KC343768 & KC343284 \\
\hline & LGMF926 & $\begin{array}{l}\text { Aspidosperma } \\
\text { tomentosum }\end{array}$ & KC343043 & KC344011 & KC343769 & KC343285 \\
\hline D. caatingaensis & CBS $141542 *$ & Tacinga inamoena & KY085927 & KY115600 & KY115603 & $\mathrm{N} / \mathrm{A}^{\mathrm{a}}$ \\
\hline D. canthii & CBS $132533 *$ & Canthium inerme & JX069864 & KC843230 & KC843120 & KC843174 \\
\hline D. cassines & СРC 21916* & Cassine peragua & KF777155 & $\mathrm{N} / \mathrm{A}^{\mathrm{a}}$ & KF777244 & $\mathrm{N} / \mathrm{A}^{\mathrm{a}}$ \\
\hline \multirow[t]{2}{*}{ D. caulivora } & CBS $127268^{*}$ & Glycine $\max$ & KC343045 & KC344013 & KC343771 & KC343287 \\
\hline & CBS 178.55 & Glycine soja & KC343046 & KC344014 & KC343772 & KC343288 \\
\hline D. celastrina & CBS $139.27^{*}$ & Celastrus scandens & KC 343047 & KC344015 & KC343773 & KC343289 \\
\hline D. charlesworthii & BRIP 54884m* & $\begin{array}{l}\text { Rapistrum } \\
\text { rugostrum }\end{array}$ & KJ197288 & KJ197268 & KJ197250 & N/A ${ }^{a}$ \\
\hline \multirow[t]{2}{*}{ D. chamaeropis } & CBS $454.81^{*}$ & $\begin{array}{l}\text { Chamaerops } \\
\text { humilis }\end{array}$ & KC343048 & KC344016 & KC343774 & KC343290 \\
\hline & CBS 753.70 & Spartium junceum & KC343049 & KC344017 & KC343775 & KC343291 \\
\hline D. cichorii & MFLUCC 17-1023* & Cichorium intybus & KY964220 & KY964104 & KY964176 & KY964133 \\
\hline D. cissampeli & $\begin{array}{l}\mathrm{CPC} 27302=\mathrm{CBS} \\
141331 *\end{array}$ & $\begin{array}{l}\text { Cissampelos } \\
\text { capensis }\end{array}$ & KX228273 & KX228384 & N/A ${ }^{a}$ & $\mathrm{~N} / \mathrm{A}^{\mathrm{a}}$ \\
\hline \multirow[t]{2}{*}{ D. citri } & CBS $135422^{*}$ & Citrus sp. & KC843311 & KC843187 & KC843071 & KC843157 \\
\hline & AR4469 & Citrus sp. & KC843321 & KC843197 & KC843081 & KC843167 \\
\hline \multirow[t]{2}{*}{ D. citriasiana } & ZJUD $30^{*}$ & Citrus unshiu & JQ954645 & KC357459 & JQ954663 & КC357491 \\
\hline & ZJUD81 & Citrus grandis & KJ490616 & KJ490437 & KJ490495 & $\mathrm{N} / \mathrm{A}^{\mathrm{a}}$ \\
\hline \multirow[t]{2}{*}{ D. citrichinensis } & ZJUD34* & Citrus unshiu & JQ954648 & KJ490396 & JQ954666 & KC357494 \\
\hline & ZJUD85 & $\begin{array}{l}\text { Fortunella } \\
\text { margarita }\end{array}$ & KJ490620 & KJ490441 & KJ490499 & $\mathrm{N} / \mathrm{A}^{\mathrm{a}}$ \\
\hline \multirow[t]{2}{*}{ D. compacta } & CGMCC $3.17536^{*}$ & Camellia sinensis & KP267854 & KP293434 & KP267928 & $\mathrm{N} / \mathrm{A}^{\mathrm{a}}$ \\
\hline & LC3078 & Camellia sinensis & KP267850 & KP293430 & KP267924 & $\mathrm{N} / \mathrm{A}^{\mathrm{a}}$ \\
\hline D. convolvuli & CBS 124654* & $\begin{array}{l}\text { Convolvulus } \\
\text { arvensis }\end{array}$ & KC343054 & KC344022 & KC343780 & KC343296 \\
\hline D. crotalariae & CBS $162.33^{*}$ & $\begin{array}{l}\text { Crotalaria } \\
\text { spectabilis }\end{array}$ & KC343056 & KC344024 & KC343782 & KC343298 \\
\hline \multirow[t]{2}{*}{ D. cucurbitae } & DAOM42078* & Cucumis sativus & KM453210 & KP118848 & KM453211 & $\mathrm{N} / \mathrm{A}^{\mathrm{a}}$ \\
\hline & CBS 136.25 & Arctium sp. & KC343031 & KC343999 & KC343757 & KC343273 \\
\hline
\end{tabular}




\begin{tabular}{|c|c|c|c|c|c|c|}
\hline D. cuppatea & CBS $117499 *$ & Aspalathus linearis & KC343057 & KC344025 & KC343783 & KC343299 \\
\hline D. cynaroidis & CBS $122676^{*}$ & Protea cynaroides & KC343058 & KC344026 & KC343784 & KC343300 \\
\hline \multirow[t]{2}{*}{ D. cytosporella } & FAU461* & Citrus limon & KC843307 & KC843221 & KC843116 & KC843141 \\
\hline & AR5149 & Citrus sinensis & KC843309 & KC843222 & KC843118 & KC843287 \\
\hline D. diospyricola & СРC $21169^{*}$ & Diospyros whyteana & KF777156 & $\mathrm{N} / \mathrm{A}^{\mathrm{a}}$ & $\mathrm{N} / \mathrm{A}^{\mathrm{a}}$ & N/A ${ }^{a}$ \\
\hline \multirow[t]{2}{*}{ D. discoidispora } & ZJUD89* & Citrus unshiu & KJ490624 & KJ490445 & KJ490503 & $\mathrm{N} / \mathrm{A}^{\mathrm{a}}$ \\
\hline & CGMCC 3.17254 & Citrus sinensis & KJ490622 & KJ490443 & KJ490501 & N/A ${ }^{a}$ \\
\hline D. dorycnii & MFLUCC 17-1015* & $\begin{array}{l}\text { Dorycnium } \\
\text { hirsutum }\end{array}$ & KY964215 & KY964099 & KY964171 & $\mathrm{N} / \mathrm{A}^{\mathrm{a}}$ \\
\hline D. elaegni-glabrae & CGMCC $3.18287 *$ & Elaeagnus glabra & KX986779 & KX999212 & KX999171 & KX999281 \\
\hline \multirow[t]{2}{*}{ D. ellipicola } & CGMCC $3.17084 *$ & Lithocarpus glabra & KF576270 & KF576291 & KF576245 & $\mathrm{N} / \mathrm{A}^{\mathrm{a}}$ \\
\hline & CGMCC 3.17085 & Lithocarpus glabra & KF576271 & KF576292 & KF576246 & $\mathrm{N} / \mathrm{A}^{\mathrm{a}}$ \\
\hline \multirow[t]{2}{*}{ D. endophytica } & CBS $133811^{*}$ & $\begin{array}{l}\text { Schinus } \\
\text { terebinthifolius }\end{array}$ & KC343065 & KC343065 & KC343791 & KC343307 \\
\hline & LGMF911 & $\begin{array}{l}\text { Schinus } \\
\text { terebinthifolius }\end{array}$ & KC343066 & KC344034 & KC343792 & KC343308 \\
\hline \multirow[t]{5}{*}{ D. eres } & AR5193* & Ulmus sp. & KJ210529 & KJ420799 & $\mathrm{KJ} 210550$ & KJ434999 \\
\hline & CBS 138598 & Ulmus sp. & $\mathrm{KJ} 210521$ & KJ420787 & KJ210545 & KJ435027 \\
\hline & CBS 439.82 & Cotoneaster sp. & FJ889450 & JX275437 & GQ250341 & JX197429 \\
\hline & DLR12A & Vitis vinifera & KJ210518 & KJ420783 & KJ210542 & KJ434996 \\
\hline & AR3669 & Pyrus pyrifolia & JQ807466 & KJ420808 & JQ807415 & KJ435002 \\
\hline D. eucalyptorum & CBS $132525^{*}$ & Eucalyptus sp. & NR120157 & $\mathrm{N} / \mathrm{A}^{\mathrm{a}}$ & N/A ${ }^{a}$ & $\mathrm{~N} / \mathrm{A}^{\mathrm{a}}$ \\
\hline \multirow[t]{6}{*}{ D. foeniculina } & CBS $111553^{*}$ & Foeniculum vulgare & KC343101 & KC344069 & KC343827 & KC343343 \\
\hline & FAU460 & Citrus limon & KC843304 & KC843218 & KC843113 & KC843138 \\
\hline & ICMP 12285 & Juglans regia & KC145853 & $\mathrm{N} / \mathrm{A}^{\mathrm{a}}$ & KC145937 & N/A \\
\hline & AR5151 & Citrus latifolia & KC843303 & KC843217 & KC843112 & KC843137 \\
\hline & CBS 187.27 & Camellia sinesis & DQ286287 & $\mathrm{JX} 275463$ & DQ286261 & KC843122 \\
\hline & CBS 123208 & Foeniculum valgare & EU814480 & JX275464 & GQ250315 & KC843125 \\
\hline $\begin{array}{l}\text { D. fraxini- } \\
\text { angustifoliae }\end{array}$ & BRIP 54781* & $\begin{array}{l}\text { Fraxinus } \\
\text { angustifolia }\end{array}$ & JX862528 & KF170920 & JX862534 & $\mathrm{N} / \mathrm{A}^{\mathrm{a}}$ \\
\hline D. fukushii & MAFF 625034 & Pyrus pyrifolia & JQ807469 & N/A & JQ807418 & $\mathrm{N} / \mathrm{A}^{\mathrm{a}}$ \\
\hline \multirow[t]{2}{*}{ D. fusicola } & CGMCC $3.17087^{*}$ & Lithocarpus glabra & KF576281 & KF576305 & KF576256 & KF576233 \\
\hline & CGMCC 3.17088 & Lithocarpus glabra & KF576263 & KF576287 & KF576238 & N/A \\
\hline D. ganjae & CBS $180.91 *$ & Cannabis sativa & KC343112 & KC344080 & KC343838 & KC343354 \\
\hline D. garethjonesii & MFLUCC 12-0542a* & Unknown dead leaf & KT459423 & KT459441 & KT459457 & KT459470 \\
\hline D. goulteri & BRIP 55657a* & Helianthus annuus & KJ197290 & KJ197270 & KJ197252 & $\mathrm{N} / \mathrm{A}^{\mathrm{a}}$ \\
\hline \multirow[t]{2}{*}{ D. gulyae } & BRIP 54025* & Helianthus annuus & JF431299 & N/A & JN645803 & $\mathrm{N} / \mathrm{A}^{\mathrm{a}}$ \\
\hline & BRIP 53158 & Helianthus annuus & JF431284 & N/A & JN645799 & $\mathrm{N} / \mathrm{A}^{\mathrm{a}}$ \\
\hline \multirow[t]{2}{*}{ D. helianthi } & CBS 592.81* & Helianthus annuus & KC343115 & KC344083 & KC343841 & KC343357 \\
\hline & CBS 344.94 & Helianthus annuus & KC343114 & KC344082 & KC343840 & KC343356 \\
\hline D. helicis & AR5211* & Hedera helix & KJ210538 & KJ420828 & KJ210559 & KJ435043 \\
\hline D. hickoriae & CBS $145.26^{*}$ & Carya glabra & KC343118 & KC344086 & KC343844 & KC343360 \\
\hline \multirow[t]{2}{*}{ D. hongkongensis } & CBS $115448^{*}$ & Dichroa febrífuga & KC343119 & KC344087 & KC343845 & KC343361 \\
\hline & ZJUD74 & Citrus unshiu & KJ490609 & KJ490430 & KJ490488 & $\mathrm{N} / \mathrm{A}^{\mathrm{a}}$ \\
\hline D. incompleta & CGMCC $3.18288^{*}$ & Camellia sinensis & KX986794 & KX999226 & KX999186 & KX999289 \\
\hline \multirow[t]{2}{*}{ D. inconspicua } & CBS 133813* & Maytenus ilicifolia & KC343123 & КC344091 & KC343849 & KC343365 \\
\hline & LGMF922 & Spondias mombin & KC343124 & KC344092 & KC 343850 & KC343366 \\
\hline D. infecunda & CBS $133812 *$ & $\begin{array}{l}\text { Schinus } \\
\text { terebinthifolius }\end{array}$ & KC343126 & KC344094 & KC 343852 & KC343852 \\
\hline
\end{tabular}




\begin{tabular}{|c|c|c|c|c|c|c|}
\hline & LGMF908 & $\begin{array}{l}\text { Schinus } \\
\text { terebinthifolius }\end{array}$ & KC343127 & KC344095 & KC343853 & KC343369 \\
\hline D. isoberliniae & CPC $22549^{*}$ & $\begin{array}{l}\text { Isoberlinia } \\
\text { angolensis }\end{array}$ & KJ869133 & KJ869245 & N/A & $\mathrm{N} / \mathrm{A}^{\mathrm{a}}$ \\
\hline D. juglandicola & CFCC 51134* & $\begin{array}{l}\text { Juglans } \\
\text { mandshurica }\end{array}$ & KU985101 & KX024634 & KX024628 & KX024616 \\
\hline \multirow[t]{2}{*}{ D. kochmanii } & BRIP 54033* & Helianthus annuus & JF431295 & $\mathrm{N} / \mathrm{A}^{\mathrm{a}}$ & JN645809 & N/A \\
\hline & BRIP 54034 & Helianthus annuus & JF431296 & N/A ${ }^{a}$ & JN645810 & $\mathrm{N} / \mathrm{A}^{\mathrm{a}}$ \\
\hline \multirow[t]{2}{*}{ D. kongii } & BRIP 54031* & Helianthus annuus & JF431301 & $\mathrm{N} / \mathrm{A}^{\mathrm{a}}$ & JN645797 & $\mathrm{N} / \mathrm{A}^{\mathrm{a}}$ \\
\hline & BRIP 54032 & Helianthus annuus & JF431300 & $\mathrm{N} / \mathrm{A}^{\mathrm{a}}$ & JN645798 & $\mathrm{N} / \mathrm{A}^{\mathrm{a}}$ \\
\hline D. litchicola & BRIP 54900* & Litchi chinensis & JX862533 & KF170925 & JX862539 & $\mathrm{N} / \mathrm{A}^{\mathrm{a}}$ \\
\hline \multirow[t]{2}{*}{ D. lithocarpus } & CGMCC $3.15175^{*}$ & Lithocarpus glabra & KC153104 & KF576311 & KC153095 & KF576235 \\
\hline & CGMCC 3.15178 & Smilax china & $\mathrm{KC} 153103$ & $\mathrm{~N} / \mathrm{A}^{\mathrm{a}}$ & KC153094 & $\mathrm{N} / \mathrm{A}^{\mathrm{a}}$ \\
\hline \multirow[t]{2}{*}{ D. longicicola } & CGMCC $3.17089^{*}$ & Lithocarpus glabra & KF576267 & KF576291 & KF576242 & $\mathrm{N} / \mathrm{A}^{\mathrm{a}}$ \\
\hline & CGMCC 3.17090 & Lithocarpus glabra & KF576268 & KF576292 & KF576243 & $\mathrm{N} / \mathrm{A}^{\mathrm{a}}$ \\
\hline \multirow[t]{2}{*}{ D. longicolla } & ATCC $60325 *$ & Glycine max & KJ590728 & KJ610883 & KJ590767 & $\mathrm{N} / \mathrm{A}^{\mathrm{a}}$ \\
\hline & FAU644 & Glycine max & KJ590730 & KJ610885 & KJ590769 & KJ612126 \\
\hline D. longispora & CBS 194.36* & Ribes sp. & KC343135 & KC344103 & KC343861 & KC343377 \\
\hline D. lonicerae & MFLUCC 17-0963* & Lonicera sp. & KY964190 & KY964073 & KY964146 & KY964116 \\
\hline \multirow[t]{2}{*}{ D. lusitanicae } & CBS 123212* & Foeniculum vulgare & KC343136 & KC344104 & KC343862 & KC343378 \\
\hline & CBS 123213 & Foeniculum vulgare & KC343137 & KC344105 & KC343863 & КC343379 \\
\hline D. macinthoshii & BRIP 55064a* & $\begin{array}{l}\text { Rapistrum } \\
\text { rugostrum }\end{array}$ & KJ197289 & KJ197269 & KJ197251 & $\mathrm{N} / \mathrm{A}^{\mathrm{a}}$ \\
\hline \multirow[t]{2}{*}{ D. mahothocarpus } & CGMCC $3.15181^{*}$ & Lithocarpus glabra & KC153096 & KF576312 & KC153087 & $\mathrm{N} / \mathrm{A}^{\mathrm{a}}$ \\
\hline & CGMCC 3.15182 & Lithocarpus glabra & KC153097 & $\mathrm{N} / \mathrm{A}^{\mathrm{a}}$ & KC153088 & $\mathrm{N} / \mathrm{A}^{\mathrm{a}}$ \\
\hline D. malorum & CAA734* & Malus domestica & KY435638 & KY435668 & KY435627 & KY435658 \\
\hline D. maritima & DAOMC $250563 *$ & Picea rubens & $\mathrm{N} / \mathrm{A}^{\mathrm{a}}$ & KU574616 & $\mathrm{N} / \mathrm{A}^{\mathrm{a}}$ & $\mathrm{N} / \mathrm{A}^{\mathrm{a}}$ \\
\hline \multirow[t]{2}{*}{ D. masirevicii } & BRIP 57892a* & Helianthus annuus & KJ197277 & KJ197257 & KJ197239 & $\mathrm{N} / \mathrm{A}^{\mathrm{a}}$ \\
\hline & BRIP 54256 & Glycine max & KJ197276 & KJ197256 & KJ197238 & $\mathrm{N} / \mathrm{A}^{\mathrm{a}}$ \\
\hline D. mayteni & CBS $133185^{*}$ & Maytenus ilicifolia & KC343139 & KC344107 & KC343865 & KC343381 \\
\hline D. maytenicola & СРC 21896* & $\begin{array}{l}\text { Maytenus } \\
\text { acuminata }\end{array}$ & KF777157 & KF777250 & $\mathrm{N} / \mathrm{A}^{\mathrm{a}}$ & $\mathrm{N} / \mathrm{A}^{\mathrm{a}}$ \\
\hline \multirow[t]{2}{*}{ D. melonis } & CBS $507.78 *$ & Glycine soja & KC343141 & KC344109 & KC343867 & KC343383 \\
\hline & FAU640 & Cucumis melo & KJ590702 & KJ610858 & KJ590741 & KJ612099 \\
\hline \multirow[t]{2}{*}{ D. middletonii } & BRIP $54884 \mathrm{e}^{*}$ & $\begin{array}{l}\text { Rapistrum } \\
\text { rugostrum }\end{array}$ & KJ197286 & KJ197266 & KJ197248 & N/A \\
\hline & BRIP 57329 & $\begin{array}{l}\text { Chrysanthemoides } \\
\text { monilifera }\end{array}$ & KJ197285 & KJ197265 & KJ197247 & $\mathrm{N} / \mathrm{A}^{\mathrm{a}}$ \\
\hline \multirow[t]{2}{*}{ D. miriciae } & BRIP 54736j* & Helianthus annuus & KJ197282 & KJ197262 & KJ197244 & N/A ${ }^{\mathrm{a}}$ \\
\hline & BRIP 55662c & Glycine max & KJ197283 & KJ197263 & KJ197245 & $\mathrm{N} / \mathrm{A}^{\mathrm{a}}$ \\
\hline D. momicola & MFLUCC 16-0113* & Prunus persica & KU557563 & KU557587 & KU557631 & KU557611 \\
\hline D. multigutullata & ZJUD98* & Citrus grandis & KJ490633 & KJ490454 & KJ490512 & $\mathrm{N} / \mathrm{A}^{\mathrm{a}}$ \\
\hline D. musigena & CBS 129519* & Musa sp. & KC343143 & KC344111 & KC343869 & KC343385 \\
\hline D. neilliae & CBS $144.27 *$ & Spiraea sp. & KC343144 & KC344112 & KC343870 & KC343386 \\
\hline D. neoarctii & CBS $109490^{*}$ & Ambrosia trifida & KC343145 & KC344113 & KC343871 & KC343387 \\
\hline \multirow[t]{2}{*}{$\begin{array}{l}\text { D. } \\
\text { neoraonikayaporum }\end{array}$} & MFLUCC $14-1136^{*}$ & Tectona grandis & KU712449 & KU743988 & KU749369 & KU749356 \\
\hline & MFLUCC 14-1133 & Tectona grandis & KU712448 & KU743987 & KU749368 & KU749355 \\
\hline D. nobilis & CBS 587.79 & Pinus pantepella & KC343153 & KC344121 & KC343879 & KC343395 \\
\hline D. nothofagi & BRIP 54801* & $\begin{array}{l}\text { Nothofagus } \\
\text { cunninghamii }\end{array}$ & JX862530 & KF170922 & JX862536 & $\mathrm{N} / \mathrm{A}^{\mathrm{a}}$ \\
\hline D. novem & CBS $127270^{*}$ & Glycine $\max$ & KC 343155 & KC344123 & KC343881 & KC343397 \\
\hline
\end{tabular}




\begin{tabular}{|c|c|c|c|c|c|c|}
\hline & CBS 127271 & Glycine max & KC343157 & KC344125 & KC343883 & КС343399 \\
\hline D. ocoteae & CBS 141330* & Ocotea obtusata & KX228293 & KX228388 & $\mathrm{N} / \mathrm{A}^{\mathrm{a}}$ & $\mathrm{N} / \mathrm{A}^{\mathrm{a}}$ \\
\hline D. oraccini & CGMCC $3.17531 *$ & Camellia sinensis & KP267863 & KP293443 & KP267937 & $\mathrm{N} / \mathrm{A}^{\mathrm{a}}$ \\
\hline D. ovalispora & ZJUD93* & Citrus limon & KJ490628 & KJ490449 & KJ490507 & $\mathrm{N} / \mathrm{A}^{\mathrm{a}}$ \\
\hline \multirow[t]{2}{*}{ D. ovoicicola } & CGMCC $3.17093^{*}$ & Citrus sp. & KF576265 & KF576289 & KF576240 & KF576223 \\
\hline & CGMCC 3.17092 & Citrus sp. & KF576264 & KF576288 & KF576239 & KF576222 \\
\hline \multirow[t]{2}{*}{ D. oxe } & CBS $133186^{*}$ & Maytenus ilicifolia & KC343164 & KC344132 & KC343890 & KC343406 \\
\hline & CBS 133187 & Maytenus ilicifolia & KC343165 & KC344133 & KC343891 & KC343407 \\
\hline D. paranensis & CBS 133184* & Maytenus ilicifolia & KC343171 & KC344139 & KC343897 & KC343413 \\
\hline D. parapterocarpi & CPC 22729* & $\begin{array}{l}\text { Pterocarpus } \\
\text { brenanii }\end{array}$ & KJ869138 & KJ869248 & $\mathrm{N} / \mathrm{A}^{\mathrm{a}}$ & $\mathrm{N} / \mathrm{A}^{\mathrm{a}}$ \\
\hline D. pascoei & BRIP 54847* & Persea americana & JX862532 & KF170924 & JX862538 & $\mathrm{N} / \mathrm{A}^{\mathrm{a}}$ \\
\hline D. passiflorae & CBS $132527 *$ & Passiflora edulis & JX069860 & $\mathrm{N} / \mathrm{A}^{\mathrm{a}}$ & $\mathrm{N} / \mathrm{A}^{\mathrm{a}}$ & $\mathrm{N} / \mathrm{A}^{\mathrm{a}}$ \\
\hline D. passifloricola & CBS 141329* & Passiflora foetida & KX228292 & KX228387 & $\mathrm{N} / \mathrm{A}^{\mathrm{a}}$ & $\mathrm{N} / \mathrm{A}^{\mathrm{a}}$ \\
\hline D. penetriteum & CGMCC $3.17532 *$ & Camellia sinensis & KP267879 & KP293459 & KP267953 & $\mathrm{N} / \mathrm{A}^{\mathrm{a}}$ \\
\hline D. perjuncta & CBS $109745^{*}$ & Ulmus glabra & KC343172 & KC344140 & KC343898 & KC343414 \\
\hline D. persiae & CBS $151.73^{*}$ & Persea gratissima & KC343173 & KC344141 & KC343899 & KC343415 \\
\hline D. pescicola & MFLUCC 16-0105* & Prunus persica & KU557555 & KU557579 & KU557623 & KU557603 \\
\hline D. phaseolorum & AR4203* & Phaseolus vulgaris & KJ590738 & KJ610893 & KJ590739 & KJ612135 \\
\hline D. phragmitis & CBS $138897 *$ & $\begin{array}{l}\text { Phragmites } \\
\text { australis }\end{array}$ & KP004445 & KP004507 & $\mathrm{N} / \mathrm{A}^{\mathrm{a}}$ & $\mathrm{N} / \mathrm{A}^{\mathrm{a}}$ \\
\hline $\begin{array}{l}\text { D. podocarpi- } \\
\text { macrophylli }\end{array}$ & CGMCC $3.18281 *$ & $\begin{array}{l}\text { Podocarpus } \\
\text { macrophyllus }\end{array}$ & KX986774 & KX999207 & KX999167 & KX999278 \\
\hline \multirow[t]{2}{*}{ D. pseudomangiferae } & CBS 101339* & Mangifera indica & KC343181 & KC344149 & KC343907 & KC343423 \\
\hline & CBS 388.89 & Mangifera indica & KC343182 & KC344150 & KC343908 & KC343424 \\
\hline \multirow[t]{2}{*}{$\begin{array}{l}\text { D. } \\
\text { pseudophoenicicola }\end{array}$} & CBS 462.69* & Phoenix dactylifera & KC 343183 & KC344151 & KC343909 & КC343425 \\
\hline & CBS 176.77 & Mangifera indica & KC 343183 & KC344151 & KC343909 & KC343425 \\
\hline D. pseudotsugae & MFLU 15-3228 & $\begin{array}{l}\text { Pseudotsuga } \\
\text { menziesii }\end{array}$ & KY964225 & KY964108 & KY964181 & KY964138 \\
\hline D. psoraleae & CBS $136412 *$ & Psoralea pinnata & KF777158 & KF777251 & KF777245 & $\mathrm{N} / \mathrm{A}^{\mathrm{a}}$ \\
\hline $\begin{array}{l}D . \\
\text { pinnatae }\end{array}$ & CBS $136413^{*}$ & Psoralea pinnata & KF777159 & KF777252 & $\mathrm{N} / \mathrm{A}^{\mathrm{a}}$ & $\mathrm{N} / \mathrm{A}^{\mathrm{a}}$ \\
\hline \multirow[t]{2}{*}{ D. pterocarpi } & MFLUCC 10-0571* & Pterocarpus indicus & JQ619899 & JX275460 & JX275416 & JX197451 \\
\hline & MFLUCC 10-575 & Pterocarpus indicus & JQ619901 & JX275462 & JX275418 & JX197453 \\
\hline \multirow[t]{2}{*}{ D. pterocarpicola } & MFLUCC $10-0580 \mathrm{a}^{*}$ & Pterocarpus indicus & JQ619887 & JX275441 & JX275403 & JX197433 \\
\hline & MFLUCC10-580b & Pterocarpus indicus & JQ619887 & JX275441 & JX275403 & JX197433 \\
\hline D. pulla & CBS 338.89* & Hedera helix & KC 343152 & KC 344120 & KC343878 & КC343394 \\
\hline D. pyracanthae & CAA483* & $\begin{array}{l}\text { Pyracantha } \\
\text { coccinea }\end{array}$ & KY435635 & KY435666 & KY435625 & KY435656 \\
\hline D. raonikayaporum & CBS $133182 *$ & Spondias mombin & KC343188 & KC344156 & KC343914 & KC343430 \\
\hline \multirow[t]{2}{*}{ D. ravennica } & MFLUCC 15-0479* & Tamarix sp. & KU900335 & KX432254 & KX365197 & N/A \\
\hline & MFLUCC 15-0480 & Tamarix sp. & KU900336 & KX377688 & KX426703 & N/A ${ }^{a}$ \\
\hline D. rhusicola & CBS $129528^{*}$ & Rhus pendulina & JF951146 & KC843205 & KC843100 & KC843124 \\
\hline D. rostrata & CFCC $50062 *$ & $\begin{array}{l}\text { Juglans } \\
\text { mandshurica }\end{array}$ & KP208847 & KP208855 & KP208853 & KP208849 \\
\hline \multirow{5}{*}{ D. rudis } & CFCC 50063 & $\begin{array}{l}\text { Juglans } \\
\text { mandshurica }\end{array}$ & KP208848 & KP208856 & KP208854 & KP208850 \\
\hline & AR3422* & $\begin{array}{l}\text { Laburnum } \\
\text { anagyroides }\end{array}$ & KC843331 & KC843177 & KC843090 & KC843146 \\
\hline & AR3654 & Rosa canina & KC843338 & KC843184 & KC843097 & KC843153 \\
\hline & ICMP 16419 & Castanea sativa & KC145904 & $\mathrm{N} / \mathrm{A}^{\mathrm{a}}$ & KC145976 & $\mathrm{N} / \mathrm{A}^{\mathrm{a}}$ \\
\hline & DA244 & Brugmansia sp. & KC843334 & KC843180 & KC843093 & KC843149 \\
\hline
\end{tabular}




\begin{tabular}{|c|c|c|c|c|c|c|}
\hline & CBS 113201 & Vitis vinifera & AY485750 & JX275454 & GQ250327 & JX197445 \\
\hline D. saccarata & CBS $116311^{*}$ & Protea repens & KC343190 & KC344158 & KC343916 & KC343432 \\
\hline D. sackstonii & BRIP 54669b* & Helianthus annuus & KJ197287 & KJ197267 & KJ197249 & $\mathrm{N} / \mathrm{A}^{\mathrm{a}}$ \\
\hline D. salicicola & BRIP 54825* & Salix purpurea & JX862531 & JX862531 & JX862537 & $\mathrm{N} / \mathrm{A}^{\mathrm{a}}$ \\
\hline \multirow[t]{2}{*}{ D. schini } & CBS 133181* & $\begin{array}{l}\text { Schinus } \\
\text { terebinthifolius }\end{array}$ & KC343191 & KC344159 & KC343917 & KC343433 \\
\hline & LGMF910 & $\begin{array}{l}\text { Schinus } \\
\text { terebinthifolius }\end{array}$ & KC343192 & KC344160 & KC343918 & KC343434 \\
\hline D. schoeni & MFLU 15-1279* & Schoenus nigricans & KY964226 & KY964109 & KY964182 & KY964139 \\
\hline \multirow[t]{2}{*}{ D. sclerotioides } & CBS 296.67* & Cucumis sativus & KC343193 & KC344161 & KC343919 & KC343435 \\
\hline & CBS 710.76 & Cucumis sativus & KC343194 & KC344162 & KC343920 & КС343436 \\
\hline \multirow[t]{2}{*}{ D. sennae } & CFCC 51636* & Senna bicapsularis & KY203724 & KY228891 & KY228885 & KY228875 \\
\hline & CFCC 51637 & Senna bicapsularis & KY203725 & KY228892 & KY228886 & KY228876 \\
\hline \multirow[t]{2}{*}{ D. sennicola } & CFCC 51634* & Senna bicapsularis & KY203722 & KY228889 & KY228883 & KY228873 \\
\hline & CFCC 51635 & Senna bicapsularis & KY203723 & KY228890 & KY228884 & KY228874 \\
\hline \multirow[t]{2}{*}{ D. serafiniae } & BRIP 55665a* & Helianthus annuus & KJ197274 & KJ197254 & KJ197236 & N/A ${ }^{a}$ \\
\hline & BRIP 54136 & Lupinus albus & KJ197273 & KJ197253 & KJ197235 & $\mathrm{N} / \mathrm{A}^{\mathrm{a}}$ \\
\hline D. siamensis & MFLUCC10-573a* & Dasymaschalon sp. & JQ619879 & JX275429 & JX275393 & $\mathrm{N} / \mathrm{A}^{\mathrm{a}}$ \\
\hline \multirow[t]{6}{*}{ D. sojae } & FAU635* & Glycine max & KJ590719 & KJ610875 & KJ590762 & KJ612116 \\
\hline & CBS 116019 & Caperonia palustris & KC343175 & KC344143 & KC343901 & KC343417 \\
\hline & FAU455 & Stokesia laevis & KJ590712 & KJ610868 & KJ590755 & KJ612109 \\
\hline & DP0601 & Glycine $\max$ & KJ590706 & KJ610862 & KJ590749 & KJ612103 \\
\hline & MAFF 410444 & Cucumis melo & KJ590714 & KJ610870 & KJ590757 & KJ612111 \\
\hline & BRIP 54033 & Helianthus annuus & JF431295 & $\mathrm{N} / \mathrm{A}^{\mathrm{a}}$ & JN645809 & $\mathrm{N} / \mathrm{A}^{\mathrm{a}}$ \\
\hline D. spartinicola & CBS $140003^{*}$ & Spartium junceum & KR611879 & $\mathrm{N} / \mathrm{A}^{\mathrm{a}}$ & $\mathrm{N} / \mathrm{A}^{\mathrm{a}}$ & $\mathrm{N} / \mathrm{A}^{\mathrm{a}}$ \\
\hline \multirow[t]{2}{*}{ D. sterilis } & CBS $136969 *$ & $\begin{array}{l}\text { Vaccinium } \\
\text { corymbosum }\end{array}$ & KJ160579 & KJ160528 & KJ160611 & KJ160548 \\
\hline & CPC 20580 & $\begin{array}{l}\text { Vaccinium } \\
\text { corymbosum }\end{array}$ & KJ160582 & KJ160531 & KJ160614 & KJ160551 \\
\hline D. stictica & CBS 370.54* & Buxus sampervirens & KC 343212 & KC344180 & KC343938 & KC343454 \\
\hline \multirow[t]{2}{*}{ D. subclavata } & ZJUD95* & Citrus sp. & KJ490630 & KJ490451 & KJ490509 & $\mathrm{N} / \mathrm{A}^{\mathrm{a}}$ \\
\hline & CGMCC 3.17253 & Citrus grandis & KJ490618 & KJ490439 & KJ490497 & $\mathrm{N} / \mathrm{A}^{\mathrm{a}}$ \\
\hline D. subordinaria & CBS 464.90* & Plantago lanceolata & KC343214 & KC344182 & KC343940 & KC343456 \\
\hline D. taoicola & MFLUCC 16-0117* & Prunus persica & KU557567 & KU557591 & KU557635 & $\mathrm{N} / \mathrm{A}^{\mathrm{a}}$ \\
\hline D. tectonae & MFLUCC $12-0777^{*}$ & Tectona grandis & KU712430 & KU743977 & KU749359 & KU749345 \\
\hline D. tectonendophytica & MFLUCC $13-0471 *$ & Tectona grandis & KU712439 & KU743986 & KU749367 & KU749354 \\
\hline D. tectonigena & MFLUCC 12-0767* & Tectona grandis & KU712429 & KU743976 & KU749371 & KU749358 \\
\hline \multirow[t]{2}{*}{ D. terebinthifolii } & CBS $133180^{*}$ & $\begin{array}{l}\text { Schinus } \\
\text { terebinthifolius }\end{array}$ & KC343216 & KC344184 & KC343942 & КC343458 \\
\hline & LGMF907 & $\begin{array}{l}\text { Schinus } \\
\text { terebinthifolius }\end{array}$ & KC343217 & KC344185 & KC343943 & КC343459 \\
\hline \multirow[t]{2}{*}{ D. ternstroemia } & CGMCC $3.15183^{*}$ & $\begin{array}{l}\text { Ternstroemia } \\
\text { gymnanthera }\end{array}$ & KC153098 & $\mathrm{N} / \mathrm{A}^{\mathrm{a}}$ & KC153089 & N/A ${ }^{a}$ \\
\hline & CGMCC 3.15184 & $\begin{array}{l}\text { Ternstroemia } \\
\text { gymnanthera }\end{array}$ & KC153099 & $\mathrm{N} / \mathrm{A}^{\mathrm{a}}$ & KC153090 & $\mathrm{N} / \mathrm{A}^{\mathrm{a}}$ \\
\hline \multirow[t]{2}{*}{ D. thunbergii } & MFLUCC10-576a* & $\begin{array}{l}\text { Thunbergia } \\
\text { laurifolia }\end{array}$ & JQ619893 & JX275449 & JX275409 & JX197440 \\
\hline & MFLUCC 10-576b & $\begin{array}{l}\text { Thunbergia } \\
\text { laurifolia }\end{array}$ & JQ619894 & JX275450 & JX275410 & JX197441 \\
\hline D. thunbergiicola & MFLUCC $12-0033^{*}$ & $\begin{array}{l}\text { Thunbergia } \\
\text { laurifolia }\end{array}$ & KP715097 & $\mathrm{N} / \mathrm{A}^{\mathrm{a}}$ & KP715098 & $\mathrm{N} / \mathrm{A}^{\mathrm{a}}$ \\
\hline D. torilicola & MFLUCC $17-1051^{*}$ & Torilis arvensis & KY964212 & KY964096 & KY964168 & KY964127 \\
\hline D. toxica & CBS 534.93* & $\begin{array}{l}\text { Lupinus } \\
\text { angustifolius }\end{array}$ & KC 343220 & KC344188 & KC343946 & KC343462 \\
\hline
\end{tabular}




\begin{tabular}{|c|c|c|c|c|c|c|}
\hline & CBS 546.93 & Lupinus sp. & KC343222 & KC344190 & КC343948 & KC343464 \\
\hline D. tulliensis & BRIP 62248a* & $\begin{array}{l}\text { Theobroma cacao } \\
\text { fruit }\end{array}$ & KR936130 & KR936132 & KR936133 & $N / A^{a}$ \\
\hline \multirow[t]{2}{*}{ D. ueckerae } & FAU656* & Cucumis melo & KJ590726 & KJ610881 & KJ590747 & KJ612122 \\
\hline & LGMF947 & Glycine max & KC343203 & KC344171 & KC343929 & KC343445 \\
\hline D. undulata & CGMCC $3.18293^{*}$ & $\begin{array}{l}\text { Leaf of unknown } \\
\text { host }\end{array}$ & KX986798 & KX999230 & KX999190 & $\mathrm{N} / \mathrm{A}^{\mathrm{a}}$ \\
\hline D. unshiuensis & ZJUD52* & Citrus sp. & KJ490587 & KJ490408 & KJ490466 & $\mathrm{N} / \mathrm{A}^{\mathrm{a}}$ \\
\hline \multirow{4}{*}{ D. vaccinii } & ZJUD50 & $\begin{array}{l}\text { Fortunella } \\
\text { margarita }\end{array}$ & KJ490585 & KJ490406 & KJ490464 & $\mathrm{N} / \mathrm{A}^{\mathrm{a}}$ \\
\hline & CBS $160.32 *$ & $\begin{array}{l}\text { Vaccinium } \\
\text { macrocarpon }\end{array}$ & AF317578 & JX270436 & GQ250326 & КС343470 \\
\hline & CBS 122116 & $\begin{array}{l}\text { Vaccinium } \\
\text { corymbosum }\end{array}$ & KC 343227 & KC344195 & KC343953 & КС343469 \\
\hline & CBS 135436 & $\begin{array}{l}\text { Vaccinium } \\
\text { corymbosum }\end{array}$ & AF317570 & KC843225 & JQ807380 & КС 849456 \\
\hline D. vangueriae & CPC 22703* & Vangueria infausta & KJ869137 & KJ869247 & $\mathrm{N} / \mathrm{A}^{\mathrm{a}}$ & $\mathrm{N} / \mathrm{A}^{\mathrm{a}}$ \\
\hline D. vawdreyi & BRIP 57887a* & Psidium guajava & KR936126 & KR936128 & KR936129 & $\mathrm{N} / \mathrm{A}^{\mathrm{a}}$ \\
\hline D. velutina & CGMCC $3.18286^{*}$ & Neolitsea sp. & KX986790 & KX999223 & KX999182 & $\mathrm{N} / \mathrm{A}^{\mathrm{a}}$ \\
\hline D. virgiliae & CMW40748* & Virgilia oroboides & KP247566 & KP247575 & $\mathrm{N} / \mathrm{A}^{\mathrm{a}}$ & $\mathrm{N} / \mathrm{A}^{\mathrm{a}}$ \\
\hline D. xishuangbanica & CGMCC $3.18282 *$ & Camellia sinensis & KX986783 & KX999216 & KX999175 & $\mathrm{N} / \mathrm{A}^{\mathrm{a}}$ \\
\hline D. yunnanensis & CGMCC $3.18289^{*}$ & Coffea sp. & KX986796 & KX999228 & KX999188 & KX999290 \\
\hline Diaporthella corylina & CBS 121124* & Corylus sp. & KC343004 & KC343972 & KC343730 & KC343246 \\
\hline
\end{tabular}

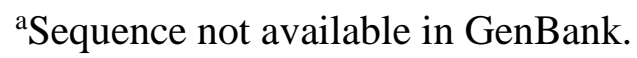

\section{Materials and methods}

The species included in Udayanga et al. (2012a) were used as the starting point. Species not included in that paper, or introduced since 2013 were found in searches of MycoBank and the mycological literature in general. All names were checked for validity, and for the existence of type cultures and relevant sequences in GenBank before they were included in the current list. New disease reports, distribution and host associations were retrieved from recent publications and from USDA fungal database (Farr \& Rossman 2016), of which are proved with molecular data.

\section{Phylogenetic analysis}

Sequences of internal transcribed spacer (ITS), translation elongation factor (tefl- $\alpha$ ), $\beta$ tubulin, CAL moduline were download from NCBI GenBank (https://www.ncbi.nlm.nih.gov/genbank/). An overview backbone phylogenetic tree for the genus Diaporthe was constructed from ITS, tefl- $\alpha, \beta$-tubulin and CAL sequence data.

Individual loci were aligned with Clustal X v. 1.81 and combined with BioEdit v. 7.0.9.0 (Hall 1999). Further alignment of the sequences was done using default settings of MAFFT v. 7 (Katoh \& Toh 2008; http://mafft.cbrc.jp/alignment/server/) and manually adjusted where necessary using BioEdit. Maximum parsimony analysis (MP) was performed with PAUP v. 4.0b10 (Swofford 2003). Gaps were treated as missing data. Trees were inferred using the heuristic search option with Tree Bisection and Reconnection branch swapping and 1000 random sequence additions. Maxtrees was set at 1000, branches of zero length were collapsed and all multiple parsimonious trees were saved. Descriptive tree statistics for parsimony (tree length, consistency index, retention index, rescaled consistency index, and homoplasy index) were calculated for trees generated under different optimality criteria.

The best model of evolution for each gene region was determined using MrModeltest v. 2.2 (Nylander 2004) and maximum likelihood analyses were performed in RAXML GUI v. 0.9b2 (Silvestro \& Michalak 2010). The RAXML analyses were run with a rapid bootstrap analysis. 


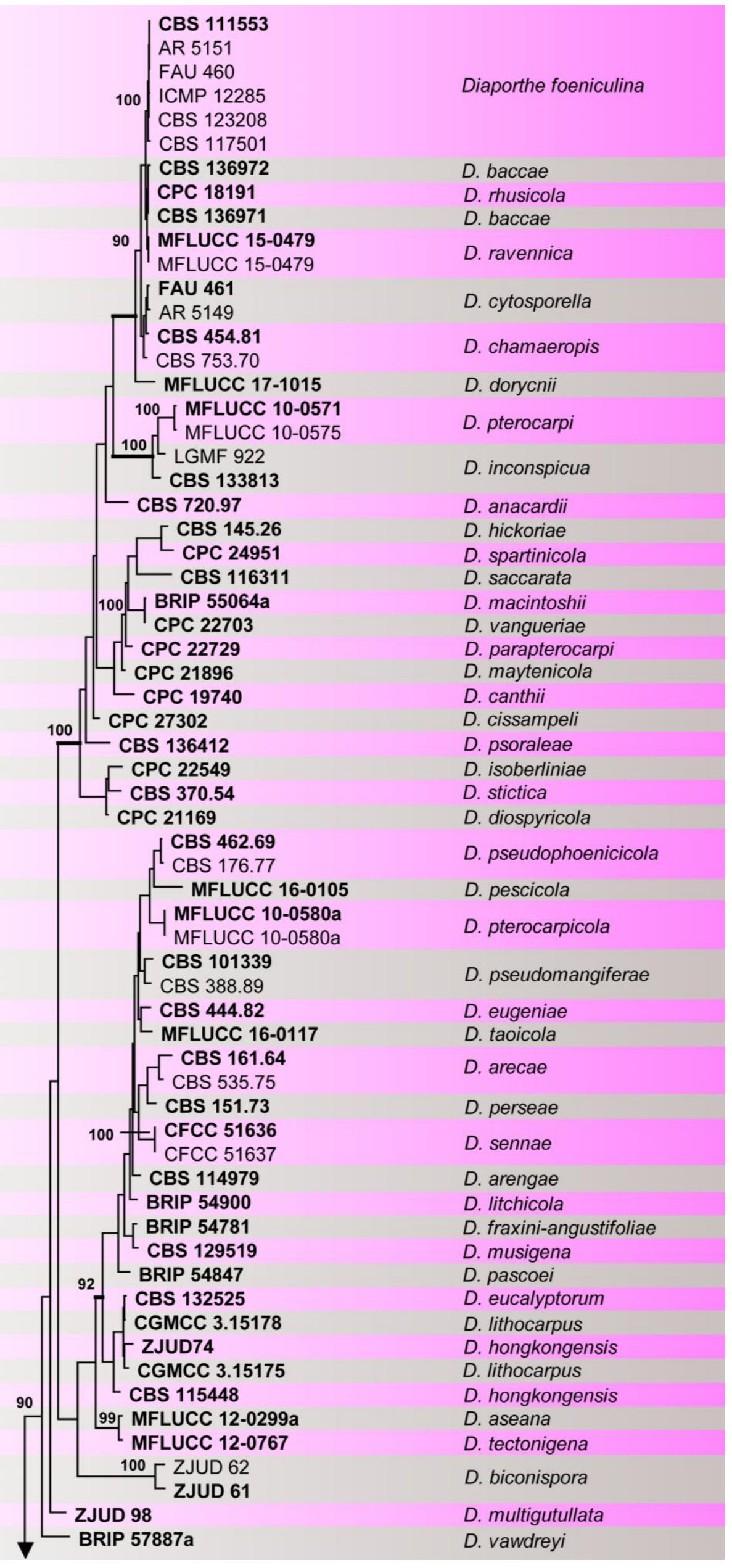




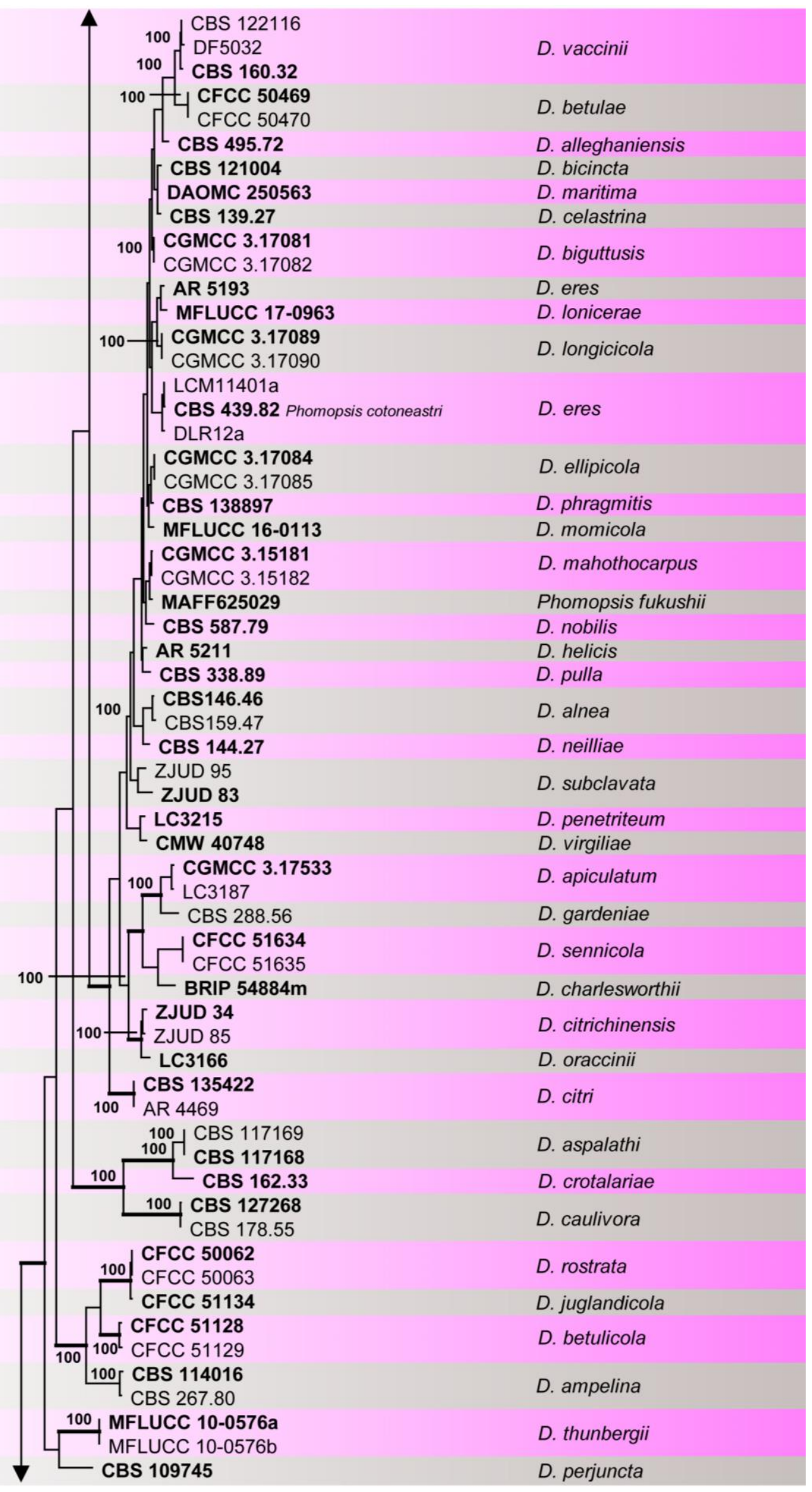




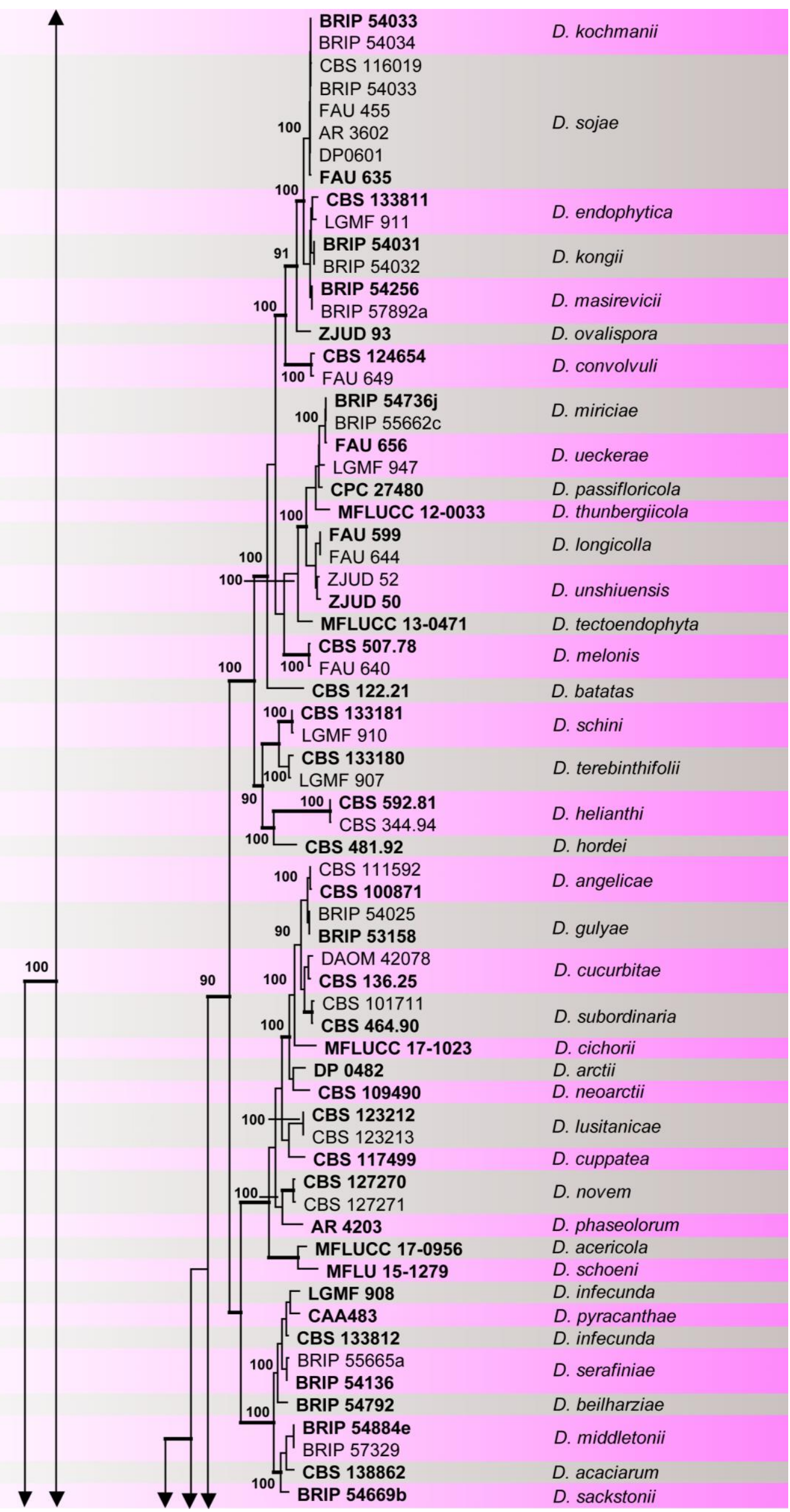




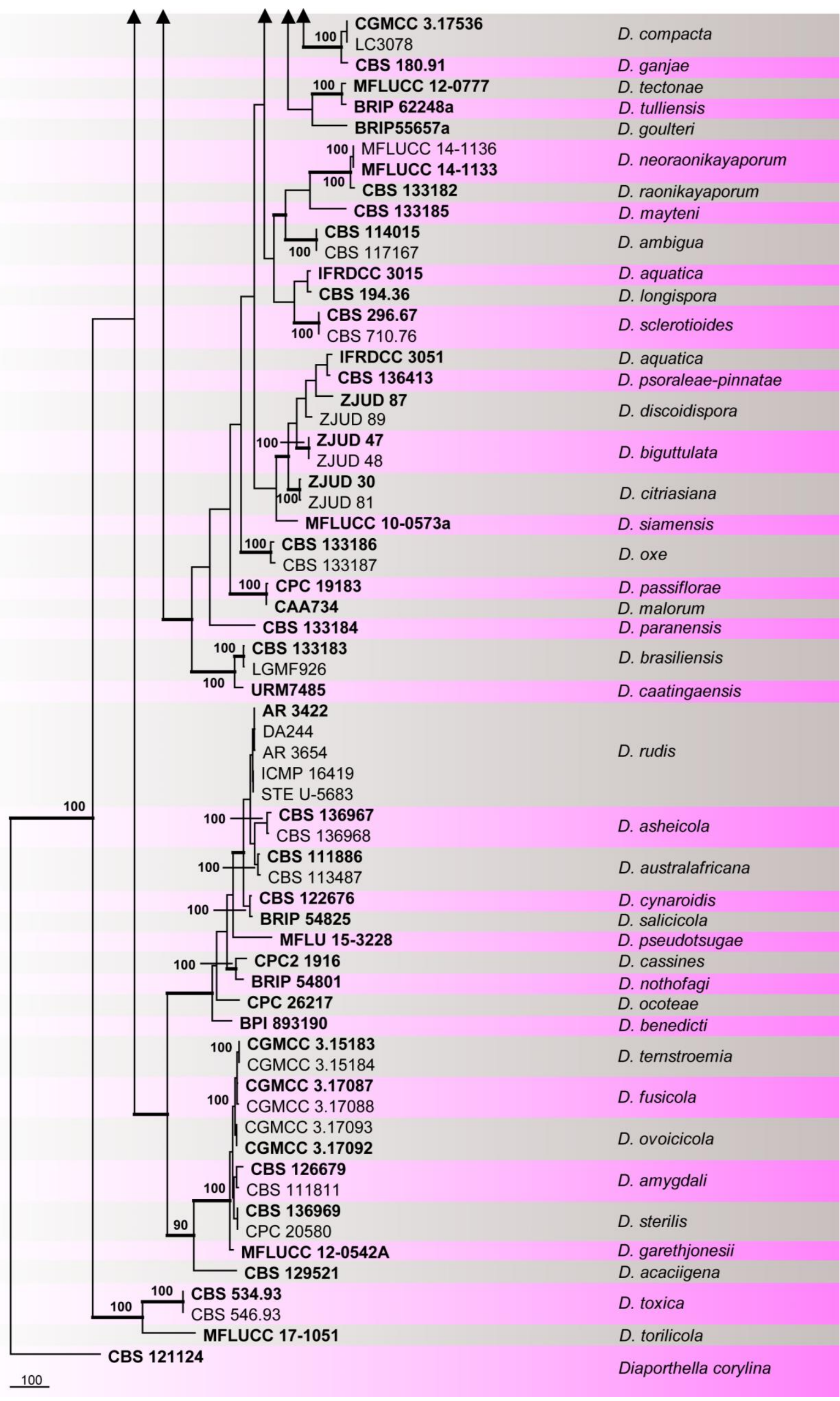


Fig. 1 - Phylogram generated from maximum parsimony analysis based on combined ITS, tefl- $\alpha$, $\beta$-tubulin and CAL dataset of the genus Diaporthe. Bootstrap support values for maximum parsimony (MP) greater than $90 \%$ are indicated near the nodes and Bayesian posterior probabilities above 0.90 are indicated in bold branches. The ex-type strains are in bold and the tree is rooted with Diaporthella corylina (CBS 121124).

Posterior probabilities (PP) were determined by Markov Chain Monte Carlo sampling (BMCMC) in MrBayes v. 3.0b4 (Ronquist \& Huelsenbeck 2003). The best-fit model of nucleotide substitution as determined above was incorporated into the analysis. Six simultaneous Markov chains were run for 1000000 generations and trees were sampled every 100th generation. The 2000 trees representing the burn-in phase of the analyses, were discarded and the remaining 8000 trees used for calculation of posterior probabilities (PP) in the majority rule consensus tree. The strains used in this study are listed in Table 1 with details of type cultures and sequences. Alignment and tree was deposited in TreeBASE (S21338).

\section{Results and Discussion}

A complete list of all species in Diaporthe that are known from culture is provided in Table 1. Where possible the ex-type isolate together with one further isolate are included. Presently 171 species are included in the genus (Fig. 1). Phomopsis fukushii is synonymized under Diaporthe fukushii.

\section{Annotated species list}

1) Diaporthe acaciarum Crous \& M.J. Wingf., Persoonia 33: 243 (2014), MycoBank MB810606. Sexual morph not reported. See Crous et al. (2014b) for illustrations and descriptions of asexual morph.

Type - Tanzania, Serengeti, on thorns of Acacia tortilis (Fabaceae), Feb. 2014, M.J. Wingfield (holotype CBS H-21994, culture ex-type CBS 138862).

Host - Acacia tortilis (Crous et al. 2014b).

Distribution - Tanzania (Crous et al. 2014b).

2) Diaporthe acaciigena Crous, Pascoe \& J. Edwards, Persoonia 26: 123 (2011), MycoBank MB560164.

Sexual morph not reported. See Crous et al. (2011) for illustrations and descriptions of asexual morph.

Type - Australia, Victoria, Otway Ranges, Anglesea, on leaves of Acacia retinodes (Fabaceae), 16 Oct. 2009, P.W. Crous, I.G. Pascoe \& J. Edwards (holotype CBS H-20581, culture ex-type CBS 129521).

Host - Acacia retinodes (Crous et al. 2011).

Distribution - Australia (Crous et al. 2011).

3) Diaporthe acericola Dissanayake, Camporesi \& K.D. Hyde, Mycosphere 8: 864 (2017), Facesoffunginumber FoF03270, Index Fungorum number IF553186.

Sexual morph not reported. See Dissanayake et al. (2017b) for illustrations and descriptions of asexual morph.

Type - Italy, Forlì-Cesena Province, San Colombano - Meldola, on dead aerial branches and samaras of Acer negundo (Sapindaceae), 22 January 2015, Erio Camporesi (holotype MFLU 153254, culture ex-type MFLUCC 17-0956).

Host - Acer negundo (Dissanayake et al. 2017b).

Distribution - Italy (Dissanayake et al. 2017b). 
4) Diaporthe acutispora Y.H. Gao \& L. Cai, IMA fungus 8: 172 (2017), MycoBank MB820679. Sexual morph not reported. See Gao et al. (2017) for illustrations and descriptions of asexual morph.

Type - China, Yunnan Province: Aini Farm, on healthy leaves of Coffea sp. (Rubiaceae), 20 Sep. 2014, W.J. Duan (holotype HMAS 247086, culture ex-type CGMCC 3.18285 = LC 6161).

Host - Coffea sp. (Gao et al. 2017).

Distribution - China (Gao et al. 2017).

5) Diaporthe alleghaniensis R.H. Arnold, Can. J. Bot. $45: 787$ (1967), MycoBank MB329848.

Sexual morph and asexual morph have been reported. See Arnold (1975) for illustrations and descriptions.

Type - Canada, Ontario, Abinger Township, Lennox and Addington Co., Vennacher, P.S.P. 10, on branch of Betula lenta (Betulaceae), 16 September 1953, R. Horner, J. Newman, A.W. Hill (holotype DAOM 45776, culture ex-type CBS 495.72).

Hosts - Betula lenta, B. papyrifera and B. pendula (Arnold 1975).

Distribution - Canada (Arnold 1975).

6) Diaporthe alnea Fuckel, Jb. nassau. Ver. Naturk. 23: 207 (1870), MycoBank MB193636.

Sexual morph and asexual morph have been reported. See Udayanga et al. (2014b) for illustrations and descriptions.

Type - Germany, on twigs of Alnus glutinosa (Betulaceae), 1894, L. Fuckel (FH, Fungi rhenani 1988, lectotype MBT178532), Hesse, Oestrich, Alnus glutinosa, 1894, L. Fuckel (BPI 615718, Isolectotype), Netherlands, on Alnus sp., June 1946, S. Truter 605 (epitype BPI 892917, culture exepitype CBS 146.46).

Hosts - Alnus glutinosa, Alnus sinuate, Alnus rugosa (Udayanga et al. 2014b).

Distribution - Czech Republic, Germany, Netherlands, USA (Udayanga et al. 2014b).

7) Diaporthe ambigua Nitschke, Pyrenomyc. Germ. 2: 311 (1870), MycoBank MB193681.

Sexual morph and asexual morph have been reported. See van Rensburg et al. (2006) for illustrations and descriptions.

Type - South Africa, Western Cape Province, from Pyrus communis (Rosaceae), deposited 2002, S. Denman (epitype CBS H-19685, culture ex-epitype CBS 114015).

Hosts - Actinidia sp. (Auger et al. 2013), Aspalathus linearis (van Rensburg et al. 2006), Foeniculum sp. (Santos \& Phillips 2009), Foeniculum vulgare, Malus domestica (Smit et al. 1996, Santos \& Phillips 2009), Malus domestica, Prunus sp. (Smit et al. 1996, Lawrence et al. 2015), Malus sylvestris (Crous et al. 2000), Prunus spp. (Smit et al. 1996, Mostert et al. 2001a), Pyrus communis (Nitschke 1867, Smit et al. 1996), Pyrus ussuriensis (Tai 1979), Salix sp. (Lawrence et al. 2015), Vaccinium sp. (Elfar et al. 2013), Vitis vinifera (van Niekerk et al. 2005, Urbez-Torres et al. 2013).

Distribution - California (Urbez-Torres et al. 2013), Chile (Auger et al. 2013, Elfar et al. 2013), China, Cuba (Tai 1979), Germany (Nitschke 1867), South Africa (Smit et al. 1996), UK (Dennis 1986), USA (Washington) (Shaw 1973).

8) Diaporthe ampelina (Berk. \& M.A. Curtis) R.R. Gomes, Glienke \& Crous, Persoonia 31: 14 (2013), MycoBank MB802922.

Sexual morph not reported. See Mostert et al. (2001a) for illustrations and descriptions of asexual morph.

Type - France, Bordeaux, Naujan-et-Postiac, on Vitis vinifera (Vitaceae, Cabernet Sauvignon grapevine), May 1998, P. Larignon (neotype PREM 56460, culture ex-neotype CBS 114016).

Hosts - Recent studies subsequent to Gomes et al. (2013) confirm the following hosts: Vitis sp. (Baumgartner et al. 2013, Urbez-Torres et al. 2013, Akgul et al. 2015, Lawrence et al. 2015). 
Distribution - Recent studies subsequent to Gomes et al. (2013) confirm the following distribution: California (Urbez-Torres et al. 2013, Lawrence et al. 2015), Turkey (Akgul et al. 2015), North America (Baumgartner et al. 2013).

9) Diaporthe amygdali (Delacr.) Udayanga, Crous \& K.D. Hyde, Fungal Diversity 56: 166 (2012), MycoBank MB800722.

Sexual morph not reported. See Diogo et al. (2010) for illustrations and descriptions of asexual morph.

Type - Portugal, Trás-os-Montes, Mirandela, on twigs of Prunus dulcis (Rosaceae), Sept. 2005, E. Diogo (epitype CBS-H 20420, culture ex-epitype CBS 126679).

Hosts - Recent studies subsequent to Gomes et al. (2013) confirm the following hosts: Camellia (Gao et al. 2016), Corylus avellana (Akay et al. 2011), Pieris japonica (Bienapfl \& Balci 2013), Prunus dulcis (Gramaje et al. 2012), Prunus persica (Zhu \& Li 2010, Dai et al. 2012), Pyrus sp. (Bai et al. 2015).

Distribution - China (Zhu \& Li 2010, Dai et al. 2012, Bai et al. 2015, Gao et al. 2016), Spain (Gramaje et al. 2012), Turkey (Akay et al. 2011), USA (Bienapfl \& Balci 2013).

10)Diaporthe anacardii (Early \& Punith.) R.R. Gomes, C. Glienke \& Crous, Persoonia 31: 15 (2013), MycoBank MB802923.

Sexual morph not reported. See Gomes et al. (2013) for illustrations and descriptions of asexual morph.

Type - East Africa, on Anacardium occidentale (Anacardiaceae), Apr. 1997, M. Puccioni (epitype CBS H-21101, culture ex-epitype CBS 720.97).

Host - Anacardium occidentale (Early \& Punithalingam 1972).

Distribution - Cuba, Guinea, Kenya, Nigeria (Early \& Punithalingam 1972).

11) Diaporthe angelicae (Berk.) Wehm., Monogr. Gen. Diaporthe Nitschke \& Segreg. 9: 204 (1933), MycoBank MB488891.

Sexual morph and asexual morph have been reported. See Castlebury et al. (2003) for illustrations and descriptions.

Type - Austria, Kaernten, Wograda, St. Margareten im Rosental, on Heracleum sphondylium (Apiaceae), Nov. 2, 2001, collected by W. Jaklitsch WJ 1842, isolated by A. Rossman (epitype BPI 842091, culture ex-epitype AR3776, CBS 111592).

Hosts - Recent studies subsequent to Castlebury et al. (2003) confirm the following hosts: Daucus carota (Menard et al. 2014), Foeniculum vulgare (Evidente et al. 2011, Rodeva \& Gabler 2011).

Distribution - Recent studies subsequent to Castlebury et al. (2003) confirm the following distribution: Bulgaria (Evidente et al. 2011, Rodeva \& Gabler 2011), France (Menard et al. 2014).

12) Diaporthe apiculata Y.H. Gao \& L. Cai, Systematics and Biodiversity 14: 106 (2016), MycoBank MB811217.

Sexual morph not reported. See Gao et al. (2016) for illustrations and descriptions of asexual morph.

Type - China, Jiangxi Province, Ganzhou, on healthy leaves of Camellia sinensis (Theaceae), 7 Sept. 2013, Y. Zhang (holotype HMAS 245777, culture ex-type CGMCC 3.17533, LC 3418).

Host - Camellia sinensis (Gao et al. 2016).

Distribution - China (Gao et al. 2016).

13)Diaporthe aquatica D.M. Hu, L. Cai \& K.D. Hyde, Mycologia 104: 1481 (2012), MycoBank MB564857.

Asexual morph not reported. Sexual morph has been reported. See Hu et al. (2012) for illustrations and descriptions. 
Type - China, Guizhou Province, Guiyang City, Tianhetan Park, on wood submerged in a small ditch, 22 May 2009, D.M. Hu (holotype IFRD 021-018, culture ex-type IFRDCC 3051; ibid (IFRD 004-014), related living culture IFRDCC 3015).

Host - submerged wood (Hu et al. 2012).

Distribution - China (Hu et al. 2012).

14) Diaporthe arctii (Lasch) Nitschke, Pyrenomyc. Germ. 2: 268 (1870), Mycobank MB189431.

Sexual morph and asexual morph have been reported. See Udayanga et al. (2015) for illustrations and descriptions.

Type - Germany, on stem of Arctium lappa (Asteraceae), 1846, Lasch (Exsiccati specimen from FH, Klotzsch, and Rabenhorst, no 1046 in Klotzschii Herbarium Vivum Mycologicum-lectotype, MBT200092). Austria, Vienna, 22nd district, Lobau, near Oelhafen, between Lobgrundstrasse and Panozzalacke, Mapping grid square 7865/1, 15 Apr. 2003, W. Jaklitsch WJ 2079 (epitype BPI 843598, culture ex-epitype CBS 139280).

Host - Arctium sp. (Udayanga et al. 2015).

Distribution - Austria, Czech Republic, France, Germany, Hungary, Poland, USA, UK (Udayanga et al. 2015).

15)Diaporthe arecae (H.C. Srivast., Zakia \& Govindar.) R.R. Gomes, C. Glienke \& Crous, Persoonia 31: 16 (2013), MycoBank MB802924.

Sexual morph not reported. See Gomes et al. (2013) for description of asexual morph.

Type - India, on fruit of Areca catechu (Arecaceae), Feb. 1964, H.C. Srivastava (isotype CBS H7808, culture ex-isotype CBS 161.64). Suriname, on fruits of Citrus sp., Oct. 1975, I. Block (CBS 535.75).

Hosts - Areca catechu (Gomes et al. 1962), Citrus sp. (Huang et al. 2015).

Distribution - China (Huang et al. 2015), India (Gomes et al. 2013), Suriname (Gomes et al. 2013).

16)Diaporthe arengae R.R. Gomes, C. Glienke \& Crous, Persoonia 31: 16 (2013), MycoBank MB802925.

Sexual morph not reported. See Gomes et al. (2013) for illustrations and descriptions of asexual morph.

Type - Hong Kong, Victoria Peak, from Arenga engleri (Arecaceae), 7 Oct. 1999, K.D. Hyde (holotype CBS H-21104, culture ex-type CBS 114979).

Host - Arenga engleri (Gomes et al. 2013).

Distribution - Hong Kong (Gomes et al. 2013).

17)Diaporthe aseana Dissanayake, Tangthirasunun \& K.D. Hyde, Fungal Diversity 80: 205 (2016), Facesoffunginumber FoF00925, Index Fungorum number IF551402.

Sexual morph not reported. See Hyde et al. (2016) for illustrations and descriptions of asexual morph.

Type - Thailand, Payao, Jam Pa Thong Waterfall, on dead leaf, 12 March 2012, N. Tangthirasunun (holotype MFLU 13-0256, culture ex-type MFLUCC 12-0299a).

Host - Unknown dead leaf (Hyde et al. 2016).

Distribution - Thailand (Hyde et al. 2016).

18)Diaporthe asheicola L. Lombard \& Crous, Phytopath. Mediterr. 53: 93 (2014), MycoBank MB807598.

Sexual morph not reported. See Lombard et al. (2014) for illustrations and descriptions of asexual morph.

Type - Chile, near Gorbea, on Vaccinium ashei (Ericaceae), Feb. 2009, A. Schilder (holotype CBS H-21513, culture ex-type CBS 136967).

Host - Vaccinium ashei (Lombard et al. 2014). 
Distribution - Chile (Lombard et al. 2014).

19)Diaporthe aspalathi E. Jansen, Castl. \& Crous, Stud. Mycol. 55: 71 (2006), MycoBank MB500803.

Sexual morph and asexual morph have been reported. See van Rensburg et al. (2006) for illustrations and descriptions.

Type - South Africa, Western Cape Province, Clanwilliam, Langebergpunt, on Aspalathus linearus (Fabaceae), J. Janse Van Rensburg, (holotype CBS H-19686, culture ex-type CBS 117169).

Hosts - Aspalathus linearus (van Rensburg et al. 2006), Glycine max (Mengistu et al. 2009, Guillin et al. 2014).

Distribution - Argentina (Guillin et al. 2014), South Africa (van Rensburg et al. 2006), USA (Mengistu et al. 2009).

20)Diaporthe australafricana Crous \& Van Niekerk, Australas. Pl. Path. 34: 33 (2005), MycoBank MB344439.

Sexual morph not reported. See Mostert et al. (2001a) for illustrations and descriptions of asexual morph.

Type - South Africa, Western Cape Province, Stellenbosch, on Riesling grapevines (Vitaceae), Nov. 1997, L. Mostert, (holotype PREM 56458, culture ex-type CBS 113487).

Hosts - Corylus sp. (Guerrero \& Perez 2013), Prunus dulcis and Salix sp. (Lawrence et al. 2015), Vaccinium sp. (Latorre et al. 2012, Elfar et al. 2013), Vitis vinifera (van Niekerk et al. 2005).

Distribution - California (Lawrence et al. 2015), Chile (Latorre et al. 2012, Elfar et al. 2013, Guerrero \& Perez 2013), South Africa (van Niekerk et al. 2005).

21)Diaporthe baccae L. Lombard, G. Polizzi \& Crous, Phytopath. Mediterr. 53: 295 (2014), MycoBank MB807599.

Sexual morph not reported. See Lombard et al. (2014) for illustrations and descriptions of asexual morph.

Type - Italy, Sicily, Catania Province, Valverde, on Vaccinium corymbosum (Ericaceae), June 2012, G. Polizzi (holotype CBS H-21514, culture ex-type CBS 136972).

Host - Vaccinium corymbosum (Lombard et al. 2014).

Distribution - Italy (Lombard et al. 2014).

22) Diaporthe batatas Harter \& E.C. Field, Phytopathology 2: 121 (1912), MycoBank MB530389.

Sexual morph and asexual morph have been reported. See Udayanga et al. (2015) for illustrations and descriptions.

Type - USA, District of Columbia, on stem and tubers of Ipomoea batatas, 1910, L.L. Harter (BPI 615746 - holotype), ibid (BPI 615747 - isotype with dry culture), on Ipomoea batatas, unknown collection dates, L.L. Harter (epitype BPI 892976, culture ex-epitype CBS 122.21, MBT 200094).

Host - Ipomoea batatas (Udayanga et al. 2015).

Distribution - USA (Udayanga et al. 2015).

23) Diaporthe beilharziae R.G. Shivas, J. Edwards \& Y.P. Tan, Fungal Diversity 61: 254 (2013), MycoBank MB802383.

Sexual morph not reported. See Tan et al. (2013) for illustrations and descriptions of asexual morph.

Type - Australia, New South Wales, Mittagong, on Indigofera australis, 30 April 1991, V.C. Beilharz (holotype VPRI 16602, includes ex-type culture).

Host - Indigofera australis (Tan et al. 2013).

Distribution - Australia (Tan et al. 2013). 
24)Diaporthe benedicti D.P. Lawrence, R. Travadon \& K. Baumgartner, Mycologia 107: 933 (2015), MycoBank MB809868.

Sexual morph not reported. See Lawrence et al. (2015) for illustrations and descriptions of asexual morph.

Type - USA, California, San Benito County northwest of Hollister, 36 $51^{\prime} 32.59^{\prime \prime} \mathrm{N}$, $121^{\circ} 26^{\prime} 44.33^{\prime \prime} \mathrm{W}, 74 \mathrm{~m}$. Salix sp. (Salicaceae) canker collected in a riparian area, 24 Jun 2011, R. Travadon No. SBen914 (holotype ATCC MYA-4970, culture ex-type BPI 893190).

Host - Salix sp. (Lawrence et al. 2015).

Distribution - USA (Lawrence et al. 2015).

25)Diaporthe betulae C.M. Tian \& X.L. Fan, Phytotaxa 269: 96 (2016), Facesoffunginumber FoF02174, Index Fungorum number IF570261.

Sexual morph not reported. See Du et al. (2016) for illustrations and descriptions of asexual morph. Type - China, Sichuan Province, Guangyuan City, Tianzhao Mountain, 32²9’22.79’N, $105^{\circ} 43^{\prime} 32.78^{\prime}$ 'E, $1422 \mathrm{~m}$ asl, on twigs and branches of Betula platyphylla, coll. X.L. Fan, 28 April 2015 (holotype BJFC-S1317, culture ex-type CFCC 50469).

Host - Betula platyphylla (Du et al. 2016).

Distribution - China (Du et al. 2016).

26)Diaporthe betulicola C.M. Tian \& Z. Du, Phytotaxa 269: 96 (2016), Facesoffunginumber FoF02173, Index Fungorum number IF570262.

Sexual morph not reported. See Du et al. (2016) for illustrations and descriptions of asexual morph. Type - China, Shaanxi Province, Ankang City, Ningshan County, Huoditang, 3326'24.15'N, $108^{\circ} 26^{\prime} 46.30^{\prime}$ 'E, $1625 \mathrm{~m}$ asl, on twigs and branches of Betula albosinensis (Betulaceae), coll. Qin Yang, 3 April 2015 (holotype BJFC-S1333, culture ex-type CFCC 51128).

Host - Betula albosinensis (Du et al. 2016).

Distribution - China (Du et al. 2016).

27)Diaporthe bicincta (Cooke \& Peck) Sacc., Syll. fung. (Abellini) 1: 622 (1882), Mycobank MB193043.

Sexual morph not reported. See Udayanga et al. (2014b) for illustrations and descriptions of asexual morph.

Type - USA, New York, Greenbush, on branch of Juglans cinerea (Juglandaceae), (NYS F 468, holotype), Tennessee, Great Smoky Mts National Park, dead wood of Juglans sp., 8 May 2006, L. Vasilyeva (epitype BPI 878472, culture ex-epitype DP0659=CBS 121004, MBT178536).

Host - Juglans sp. (Udayanga et al. 2014b).

Distribution - Canada, USA (Udayanga et al. 2014b).

28) Diaporthe biconispora F. Huang, K.D. Hyde \& H.Y. Li, Fungal Biology 119: 338 (2015), MycoBank MB810578.

Sexual morph not reported. See Huang et al. (2015) for illustrations and descriptions of asexual morph.

Type - China, Fujian Province, Zhangzhou, isolated from a healthy branch of Citrus grandis (Rutaceae), June 2011, F. Huang (holotype ZJUD62H, culture ex-type CGMCC 3.17252).

Host - Citrus grandis (Huang et al. 2015).

Distribution - China (Huang et al. 2015).

29)Diaporthe biguttulata F. Huang, K.D. Hyde \& H.Y. Li, Fungal Biology 119: 338 (2015), MycoBank MB810579.

Sexual morph not reported. See Huang et al. (2015) for illustrations and descriptions of asexual morph. 
Type - China, Yunnan Province, Ruili, Mengdian, on non-symptomatic branch of Citrus limon (Rutaceae), July 2011, F. Huang, (holotype ZJUD47H, culture ex-type CGMCC 3.17248).

Host - Citrus limon (Huang et al. 2015).

Distribution - China (Huang et al. 2015).

30)Diaporthe biguttusis Y.H. Gao \& L. Cai, Fungal Biology 119: 305 (2015), MycoBank MB805929.

Sexual morph not reported. See Gao et al. (2015) for illustrations and descriptions of asexual morph.

Type - China, Zhejiang Province, Gutianshan Nature Reserve, on leaves of Lithocarpus glabra (Fagaceae), 15 Aug. 2010, W. Sun (holotype HMAS 244838, culture ex-type CGMCC 3.17081, LC 1106).

Host - Lithocarpus glabra (Gao et al. 2015).

Distribution - China (Gao et al. 2015).

31)Diaporthe brasiliensis R.R. Gomes, C. Glienke \& Crous, Persoonia 31: 17 (2013), MycoBank MB802926.

Sexual morph not reported. See Gomes et al. (2013) for illustrations and descriptions of asexual morph.

Type - Brazil, Rio de Janeiro, endophytic species isolated from leaf of Aspidosperma tomentosum (popular name Peroba-do-campo, Apocynaceae), July 2007, K. Rodriguez (holotype CBS H-21100, culture ex-type CBS 133183).

Host - Aspidosperma tomentosum (Gomes et al. 2013).

Distribution - Brazil (Gomes et al. 2013).

32) Diaporthe caatingaensis Bezerra, Paiva, Silva, Souza-Motta \& Crous, Persoonia 37: 271 (2016), MycoBank MB818928.

Sexual morph not reported. See Crous et al. (2016) for illustrations and descriptions of asexual morph.

Type - Brazil, Pernambuco state, Itaíba municipality, Curral Velho farm, as endophyte from Tacinga inamoena (Cactaceae), Sept. 2013, J.D.P. Bezerra (holotype URM 90021, culture ex-type CBS 141542).

Host - Tacinga inamoena (Crous et al. 2016).

Distribution - Brazil (Crous et al. 2016).

33) Diaporthe canthii Crous, Persoonia 28: 159 (2012), MycoBank MB800376.

Sexual morph not reported. See Crous et al. (2012) for illustrations and descriptions of asexual morph.

Type - South Africa, Western Cape Province, Kirstenbosch Botanical Garden, on leaves of Canthium inerne ('Gewone bokdrol' in Afrikaans) (Rubiaceae), 30 July 2011, P.W. Crous, (holotype CBS H-20960, culture ex-type CBS 132533).

Host - Canthium inerne (Crous et al. 2012).

Distribution - South Africa (Crous et al. 2012).

34) Diaporthe cassines Crous, Persoonia 31: 257 (2013), MycoBank MB805857.

Sexual morph not reported. See Crous et al. (2013) for illustrations and descriptions of asexual morph.

Type - South Africa, Western Cape Province, Kirstenbosch Botanical Garden, on leaves of Cassine peragua (Celastraceae), 29 Dec. 2012, P.W. Crous (holotype CBS H-21451, culture ex-type CBS 136440).

Host - Cassine peragua (Crous et al. 2013).

Distribution - South Africa (Crous et al. 2013). 
35) Diaporthe caulivora (Athow \& Caldwell) J.M. Santos, Vrandečić \& A.J.L. Phillips, Persoonia 27: 13 (2011), MycoBank MB518520.

Sexual morph reported. See Santos et al. (2011) for illustrations and descriptions of sexual morph. Type - Croatia, Osijek, on soybean stem (Glycine max, Fabaceae), Sept. 2005, K. Vrandečić, (neotype CBS H-20461, culture ex-neotype CBS 127268).

Hosts - Abutilon theophrasti (Vrandečić et al. 2005), Glycine max (Pioli et al. 2001, Bradley \& Li 2006, Costamilan et al. 2008, Grijalba \& Ridao 2012, 2014, Sun et al. 2012, Chiesa et al. 2013, Cummings \& Bergstrom 2015), Vigna sp. (Sato et al. 2014).

Distribution - Argentina (Pioli et al. 2001, Grijalba Ridao 2012, 2014, Chiesa et al. 2013), Brazil (Costamilan et al. 2008), Canada (Ginns 1986), Croatia (Vrandečić et al. 2005), Italy (Zhang et al. 1997), Japan (Sato et al. 2014), Korea (Sun et al. 2012), USA (Bradley \& Li 2006, Cummings \& Bergstrom 2015).

36) Diaporthe celastrina Ellis \& Barthol., J. Mycol. 8: 173 (1902), Mycobank MB180525.

Sexual morph not reported. See Udayanga et al. (2014b) for illustrations and descriptions of asexual morph.

Type - USA, Kansas, Clyde, Celastrus scandens (Celastraceae), 18 May 1901, E. Bartholomew 2856 (holotype BPI 615293). USA, on Celastrus scandens, September 1927, L.E. Wehmeyer (epitype BPI 892915, culture ex-epitype CBS 139.27).

Host - Celastrus sp. (Udayanga et al. 2014b).

Distribution - USA (Udayanga et al. 2014b).

37) Diaporthe charlesworthii R.G. Shivas, S.M. Thompson \& Y.P. Tan, Persoonia 35: 43 (2015), MycoBank MB808668.

Sexual morph not reported. See Thompson et al. (2015) for illustrations and descriptions of asexual morph.

Type - Australia, Queensland, Gatton, from stem of Rapistrum rugosum (Brassicaceae), 24 Nov. 2011, S.M. Thompson T12757Z (holotype BRIP 54884m, includes ex-type culture).

Host - Rapistrum rugostrum (Thompson et al. 2015).

Distribution - Australia (Thompson et al. 2015).

38) Diaporthe chamaeropis (Cooke) R.R. Gomes, C. Glienke \& Crous, Persoonia 31: 18 (2013), MycoBank MB802927.

Sexual morph not reported. See Gomes et al. (2013) for illustrations and descriptions of asexual morph.

Authentic strain - Croatia, Rab, slope behind Hotel 'Imperial', on dead branch of Spartium junceum, July 1970, J.A. von Arx (CBS 753.70).

Hosts - Chamaerops humilis, Spartium junceum (Gomes et al. 2013), Pistacia vera (Chen et al. 2014), Salix sp., Vitis vinifera (Lawrence et al. 2015).

Distribution - California (Chen et al. 2014, Lawrence et al. 2015), Croatia, Greece (Gomes et al. 2013).

39)Diaporthe cichorii Dissanayake, Camporesi \& K.D. Hyde, Mycosphere 8: 864 (2017), Facesoffunginumber FoF03271, Index Fungorum number IF553187.

Sexual morph not reported. See Dissanayake et al. (2017b) for illustrations and descriptions of asexual morph.

Type - Italy, Forlì-Cesena Province, Santa Sofia, on dead aerial stem of Cichorium intybus (Asteraceae), 17 July 2016, Erio Camporesi (holotype MFLU 16-2168, culture ex-type MFLUCC 17-1023).

Host - Cichorium intybus (Dissanayake et al. 2017b).

Distribution - Italy (Dissanayake et al. 2017b). 
40) Diaporthe cissampeli Crous \& Roets, Persoonia 36: 361 (2016), MycoBank MB817059.

Sexual morph not reported. See Crous et al. (2016) for illustrations and descriptions of asexual morph.

Type - South Africa, Western Cape Province, Robben Island, on leaves and twigs of Cissampelos capensis (Menispermaceae), May 2015, P.W. Crous \& F. Roets (holotype CBS H-22628, culture ex-type CBS 141331)

Host - Cissampelos capensis (Crous et al. 2016).

Distribution - South Africa (Crous et al. 2016).

41) Diaporthe citri (H.S. Fawc.) F.A. Wolf, J. Agric. Res., 33: 625 (1926), Mycobank MB260952. Sexual morph and asexual morph have been reported. See Udayanga et al. (2014a) for illustrations and descriptions.

Type - USA, Florida, Lake Alfred, Ana, on twigs of Citrus sp. (Rutaceae), 26 Apr. 2000, L.W. Timmer, (type of Phomopsis citri proposed for conservation in Rossman et al. (2013) (holotype BPI 892456, culture ex-type CBS 135422).

Host - Recent studies subsequent to Udayanga et al. (2014a) confirm the following hosts: Mikania glomerata (Polonio et al. 2015).

Distribution - Recent studies subsequent to Udayanga et al. (2014a) confirm the following distribution - Brazil (Polonio et al. 2015).

42)Diaporthe citriasiana F. Huang, K.D. Hyde \& Hong Y. Li, Fungal Diversity 61: 246 (2013), MycoBank MB803202.

Sexual morph not reported. See Huang et al. (2013) for illustrations and descriptions of asexual morph.

Type - China, Shaanxi Province, Chenggu, on dead wood of Citrus unshiu (Rutaceae), March 2011, F. Huang (holotype ZJUD30H, culture ex-type CGMCC3.15224).

Host - Citrus sp. (Huang et al. 2013, 2015).

Distribution - China (Huang et al. 2013, 2015).

43)Diaporthe citrichinensis F. Huang, K.D. Hyde \& Hong Y. Li, Fungal Diversity 61: 247 (2013), MycoBank MB803203.

Sexual morph not reported. See Huang et al. (2013) for illustrations and descriptions of asexual morph.

Type - China, Shaanxi province, Chenggu, on dead wood of Citrus unshiu (Rutaceae), March 2011, F. Huang (holotype ZJUD34H, culture ex-type CGMCC3.15225).

Host - Citrus sp. (Huang et al. 2013, 2015).

Distribution - China (Huang et al. 2013, 2015).

44)Diaporthe compacta Y.H. Gao \& L. Cai, Systematics and Biodiversity 14: 110 (2016), Mycobank MB811219.

Sexual morph not reported. See Gao et al. (2016) for illustrations and descriptions of asexual morph.

Type - China, Jiangxi Province, Chongyi country, on healthy leaves of Camellia sinensis (Theaceae), 24 Apr. 2013, F. Liu, (holotype HMAS 245778, culture ex-type CGMCC 3.17536).

Host - Camellia sinensis (Gao et al. 2016).

Distribution - China (Gao et al. 2016).

45) Diaporthe convolvuli (Ormeno-Nuñez, Reeleder \& A.K. Watson) R.R. Gomes, C. Glienke \& Crous, Persoonia 31: 19 (2013), MycoBank MB802928.

Sexual morph not reported. See Ormeno-Nuñez et al. (1988) and Gomes et al. (2013) for illustrations and descriptions of asexual morph. 
Type - Turkey, isolated from leaves with anthracnose on Convolvulus arvensis (Convolvulaceae), D. Berner (holotype, culture ex-type CBS 124654 = DP 0727).

Host - Convolvulus arvensis (Ormeno-Nuñez et al. 1988, Gomes et al. 2013).

Distribution - Turkey (Ormeno-Nuñez et al. 1988, Gomes et al. 2013).

46) Diaporthe crotalariae G.F. Weber, Phytopathology 23: 602 (1933), Mycobank MB262360.

Asexual morph not reported. See Weber (1933) for illustrations and descriptions of sexual morph.

Type - USA, on Crotalaria spectabilis (Fabaceae), Oct. 1933, G.F. Weber (holotype, culture extype CBS 162.33).

Host - Crotalaria spectabilis (Weber 1933).

Distribution - USA (Weber 1933).

47)Diaporthe cucurbitae (McKeen) Udayanga \& Castl. Fungal Biology 119: 395 (2014), Mycobank MB812623.

Sexual morph not reported. See Udayanga et al. (2015) for illustrations and descriptions of asexual morph.

Type - Canada, Ontario, Leamington, on cucumber stems, 1951, C.D. McKeen (DAOM 41000 holotype), Leamington, on seeds of Cucumis sp., Apr. 1956, C.D. McKeen, MEE M-151 (epitype BPI 892977, culture ex-epitype DAOM 42078).

Hosts - Arctium sp., Cucumis sp. (Udayanga et al. 2015).

Distribution - Canada (Udayanga et al. 2015).

48) Diaporthe cuppatea (E. Jansen, Lampr. \& Crous) Udayanga, Crous \& K.D. Hyde, Fungal Diversity 56: 166 (2012), MycoBank MB500804.

Sexual morph not reported. See van Rensburg et al. (2006) for illustrations and descriptions of asexual morph.

Type - South Africa, Western Cape Province, Clanwilliam, Kossakse werf, on Aspalathus linearis (Fabaceae), J. Janse van Rensburg, (holotype CBS H-19687, culture ex-type CBS 117499).

Host - Aspalathus linearis (van Rensburg et al. 2006).

Distribution - South Africa (van Rensburg et al. 2006).

49) Diaporthe cynaroidis Marinc., M.J. Wingf. \& Crous, CBS Diversity Ser. (Utrecht) 7: 39 (2008), Mycobank MB506209.

Sexual morph not reported. See Marincowitz et al. (2008) for illustrations and descriptions of asexual morph.

Type - South Africa, Western Cape Province, on leaf litter of Protea cynaroides (Proteaceae), 26 June 2000, S. Marincowitz (holotype, culture ex-type CBS 122676).

Host - Protea cynaroides (Marincowitz et al. 2008).

Distribution - South Africa (Marincowitz et al. 2008).

50) Diaporthe cytosporella (Penz. \& Sacc.) D. Udayanga \& L.A. Castlebury, Persoonia 32: 95 (2014), MycoBank MB803986.

Sexual morph not reported. See Udayanga et al. (2014a) for illustrations and descriptions of asexual morph.

Type - Italy, Rome, Modena, on Citrus limonia (Rutaceae), Jan. 1886 (holotype of Phoma cytosporella BPI 798526). Spain, on Citrus limon, M.E. Palm (epitype BPI 892459, culture exepitype CBS 137020).

Hosts - Citrus limon, C. sinensis and Vitis vinifera (Udayanga et al. 2014a).

Distribution - Spain, Italy, United States (California) (Udayanga et al. 2014a).

51) Diaporthe diospyricola Crous, Persoonia 31: 255 (2013), MycoBank MB805856. 
Sexual morph is not reported. See Crous et al. (2013) for illustrations and descriptions of asexual morph.

Type - South Africa, Western Cape Province, Kirstenbosch Botanical Garden, on leaves of Diospyros whyteana (Ebenaceae), 30 July 2012, P.W. Crous (holotype CBS H-21450, culture extype CBS 136552)

Host - Diospyros whyteana (Crous et al. 2013).

Distribution - South Africa (Crous et al. 2013).

52)Diaporthe discoidispora F. Huang, K.D. Hyde \& H.Y. Li, Fungal Biology 119: 341 (2015), Mycobank MB810580.

Sexual morph not reported. See Huang et al. (2015) for illustrations and descriptions of asexual morph.

Type - China, Jiangxi Province, Nankang city, Tandong, on non-symptom twig of Citrus unshiu (Rutaceae), July 2011, F. Huang (holotype ZJUD89H, culture ex-type CGMCC 3.17255).

Hosts - Camellia sinensis (Gao et al. 2016), Citrus unshiu (Huang et al. 2015).

Distribution - China (Huang et al. 2015, Gao et al. 2016).

53)Diaporthe dorycnii Dissanayake, Camporesi \& K.D. Hyde, Mycosphere 8: 867 (2017), Facesoffunginumber FoF03272, Index Fungorum number IF553188.

Sexual morph not reported. See Dissanayake et al. (2017b) for illustrations and descriptions of asexual morph.

Type - Italy, Forlì-Cesena Province, Fiumicello di Premilcuore, on dead aerial stem of Dorycnium hirsutum (Fabaceae), 2 May 2016, Erio Camporesi (holotype MFLU 16-1322, culture ex-type MFLUCC 17-1015).

Host - Dorycnium hirsutum (Dissanayake et al. 2017b).

Distribution - Italy (Dissanayake et al. 2017b).

54)Diaporthe elaeagni-glabrae Y.H. Gao \& L. Cai, IMA fungus 8: 172 (2017), MycoBank MB820680.

Sexual morph not reported. See Gao et al. (2017) for illustrations and descriptions of asexual morph.

Type - China, Jiangxi Province, on diseased leaves of Elaeagnus glabra (Elaeagnaceae), 5 Sep. 2013, Y.H. Gao (holotype HMAS 247089, culture ex-type CGMCC 3.18287 = LC 4802).

Host - Elaeagnus glabra (Gao et al. 2017).

Distribution - China (Gao et al. 2017).

55)Diaporthe ellipicola Y.H. Gao \& L. Cai, Fungal Biology 119: 303 (2015), MycoBank MB805927.

Sexual morph not reported. See Gao et al. (2015) for illustrations and descriptions of asexual morph.

Type - China, Zhejiang Province, Gutianshan Nature Reserve, N29'20', E118'14', on leaves of Lithocarpus glabra (Fagaceae), 15 Aug. 2010, W. Sun (holotype HMAS 244836, culture ex-type CGMCC 3.17084).

Host - Lithocarpus glabra (Gao et al. 2015).

Distribution - China (Gao et al. 2015).

56) Diaporthe endophytica R.R. Gomes, C. Glienke \& Crous, Persoonia 31: 20 (2013), MycoBank MB802929.

Sexual morph not reported. See Gomes et al. 2013 for descriptions of asexual morph.

Type - Brazil, endophytic in leaf of Schinus terebinthifolius (Anacardiaceae), July 2007, J. Lima (LGMF 911 = CPC 20287, LGMF 919 = CPC 20295), (holotype CBS H-21107, culture ex-type CBS 133811) 
Hosts - Citrus sp. (Huang et al. 2015), Glycine max, Maytenus ilicifolia, Schinus terebinthifolius (Gomes et al. 2013).

Distribution - Brazil (Gomes et al. 2013), China (Huang et al. 2015).

57) Diaporthe eres Nitschke, Pyrenomyc. Germ. 2: 245 (1870), Mycobank MB172054.

Sexual morph and asexual morph have been reported. See Udayanga et al. (2014b) for illustrations and descriptions.

Type - Germany, Nordrhein-Westfalen, Munsterland, Munster Botanical Gardens, on twigs of Ulmus sp. (Ulmaceae), June 1865, T. Nitschke, (B 70 0009145, lectotype, MBT178528, isolectotypes ex herb. Munster; B 70 0009146, B 70 0009147), Carpinion forest, on dead, attached, corticated twigs of Ulmus laevis, 5 January 2013, R. Jarling, comm. R. Schumacher (epitype BPI 892912, culture ex-epitype AR5193).

Hosts - Recent studies subsequent to Udayanga et al. (2014b) confirm the following hosts: Camellia sinensis (Gao et al. 2016), Citrus sp. (Huang et al. 2015), Fraxinus excelsior (Kowalski et al. 2016), Glycine max (Petrovic et al. 2015), Pyrus sp. (Bai et al. 2015), Salix sp. (Lawrence et al. 2015), Tilia cordata (Stravinskiene et al. 2015), Vitis vinifera (Dissanayake et al. 2015, Lawrence et al. 2015, Cinelli et al. 2016).

Distribution - Recent studies subsequent to Udayanga et al. (2014b) confirm the following distribution: California (Lawrence et al. 2015), China (Bai et al. 2015, Dissanayake et al. 2015, Huang et al. 2015, Gao et al. 2016), Italy (Cinelli et al. 2016), Lithuania (Stravinskiene et al. 2015), Poland (Kowalski et al. 2016), Serbia (Petrovic et al. 2015).

58) Diaporthe eucalyptorum Crous \& R.G. Shivas, Persoonia 28: 153 (2012), MycoBank MB800374.

Sexual morph not reported. See Crous et al. (2012) for illustrations and descriptions of asexual morph.

Type - Australia, Queensland, Cairns Road to Atherton Giles Highway, on leaves of Eucalyptus sp. (Myrtaceae), 16 Aug. 2009, P.W. Crous (holotype CBS H-20958, culture ex-type CBS 132525).

Host - Eucalyptus sp. (Crous et al. 2012).

Distribution - Australia (Crous et al. 2012).

59) Diaporthe foeniculina (Sacc.) D. Udayanga \& L.A. Castlebury, Persoonia 32: 95 (2014), MycoBank MB803929.

Sexual morph and asexual morph have been reported. See Phillips (2003) and Udayanga et al. (2014a) for illustrations and descriptions.

Type - Portugal, Madeira, Serra da Agua, at base of 2-yr-old stem of Foeniculum vulgare (Apiaceae), Aug. 2001, A.J.L. Phillips (epitype LISE 94791, culture ex-epitype CBS).

Hosts - Recent studies subsequent to Udayanga et al. (2014a) confirm the following hosts: Castanea sativa (Annesi et al. 2016), Salix sp. (Lawrence et al. 2015).

Distribution - Recent studies subsequent to Udayanga et al. (2014a) confirm the following distribution: California (Lawrence et al. 2015), Italy (Annesi et al. 2016).

60) Diaporthe fraxini-angustifoliae R.G. Shivas, Jacq. Edwards \& Y.P. Tan, Fungal Diversity 61: 255 (2013), MycoBank MB802384.

Sexual morph not reported. See Tan et al. (2013) for illustrations and descriptions of asexual morph.

Type - Australia, Victoria, on Fraxinus angustifolia (Oleaceae), 31 Oct. 1979, L. Smith (holotype VPRI 10911, includes ex-type culture), isotype BRIP 54781.

Host - Fraxinus angustifolia (Tan et al. 2013).

Distribution - Australia (Tan et al. 2013). 
61) Diaporthe fukushii (Tanaka \& S. Endô) Dissanayake, A.J.L. Phillips \& K.D. Hyde, comb. nov. Index Fungorum number IF553773.

Basionym - Phomopsis fukushii Tanaka \& S. Endô, J. Pl. Prot. Japan 13: 1 (1927), Index Fungorum number IF265419.

See Gomes et al. (2013) for details.

Type - Japan, Ibaraki, on Pyrus pyrifolia (Rosaceae), August 1994, S. Kanematsu, (neotype BPI 892933, culture ex-neotype MAFF625034=AR3672; MBT178531).

Host - Pyrus pyrifolia (Gomes et al. 2013).

Distribution - New Zealand (Gomes et al. 2013).

62)Diaporthe fusicola Y.H. Gao \& L. Cai, Fungal Biology 119: 304 (2015), MycoBank MB805928.

Sexual morph not reported. See Gao et al. (2015) for illustrations and descriptions of asexual morph.

Type - China, Zhejiang Province, Gutianshan Nature Reserve, N29'20', E118'14', on leaves of Lithocarpus glabra (Fagaceae), 15 Aug. 2010, W. Sun (holotype HMAS 244837, culture ex-type CGMCC 3.17087).

Host - Lithocarpus glabra (Gao et al. 2015).

Distribution - China (Gao et al. 2015).

63)Diaporthe ganjae (McPartl.) R.R. Gomes, Glienke \& Crous, Persoonia 31: 22 (2013), MycoBank MB802932.

Sexual morph not reported. See Gomes et al. (2013) for illustrations and descriptions of asexual morph.

Type - USA, Illinois, Hannah City, dead leaf of Cannabis sativa (Cannabaceae), deposited Mar. 1991, J.M. McPartland (holotype HA 10987, culture ex-type CBS 180.91).

Host - Cannabis sativa (Gomes et al. 2013).

Distribution - USA (Gomes et al. 2013).

64)Diaporthe garethjonesii Dissanayake, Tangthirasunun \& K.D. Hyde, Fungal Diversity 80: 209 (2016), Facesoffunginumber FoF00926, Index Fungorum number IF551403.

Sexual morph not reported. See Hyde et al. (2016) for illustrations and descriptions of asexual morph.

Type - Thailand, Kanjanaburi, on dead leaf, 5 May 2012, Jayarama Bhat (holotype MFLU 130261, culture ex-type MFLUCC 12-0542a).

Host - Unknown dead leaf (Hyde et al. 2016).

Distribution - Thailand (Hyde et al. 2016).

65) Diaporthe goulteri R.G. Shivas, S.M. Thompson \& Y.P. Tan, Persoonia 35: 43 (2015), MycoBank MB808669.

Sexual morph not reported. See Thompson et al. (2015) for illustrations and descriptions of asexual morph.

Type - Australia, Queensland, Ryeford, from a seed of Helianthus annuus (Asteraceae), 15 Feb. 2011, S.M. Thompson, T12996A (holotype BRIP 55657a, includes ex-type culture).

Host - Helianthus annuus (Thompson et al. 2015).

Distribution - Australia (Thompson et al. 2015).

66) Diaporthe gulyae R.G. Shivas, S.M. Thomps. \& A.J. Young, Persoonia 27: 85 (2011), MycoBank MB561569.

Sexual morph not reported. See Thompson et al. (2011) for illustrations and descriptions of asexual morph. 
Type - Australia, Queensland, Ryeford near Clifton, on Helianthus annuus (Asteraceae), 7, 29 Nov. 2010, S.M. Thompson (holotype BRIP 54025, includes ex-type culture).

Host - Helianthus annuus (Thompson et al. 2011, Mathew et al. 2015a, b).

Distribution - Australia (Thompson et al. 2011), Canada (Mathew et al. 2015a), USA (Mathew et al. 2015b).

67) Diaporthe helianthi Munt. Cvetk., Mihaljč. \& M. Petrov, Nova Hedwigia 34: 433 (1981), Mycobank MB111402.

Sexual morph not reported. See Gao et al. (2017) for illustrations and descriptions of asexual morph.

Type - Serbia, Vojvodina, overwintering stem on Helianthus annuus (Asteraceae), 1980, M. Muntañola-Cvetkovic (holotype CBS H-1540, culture ex-type CBS 592.81).

Host - Helianthus annuus (Gomes et al. 2013).

Distribution - Worldwide (Gomes et al. 2013).

68)Diaporthe helicis Niessl, Verh. nat. Ver. Brünn 14: 210 (1876), Mycobank MB156620.

Sexual morph not reported. See Udayanga et al. (2014b) for illustrations and descriptions of asexual morph.

Type - Germany, Saxony, Islebiam, on vines of Hedera helix (Araliaceae), June 1875, J. Kunze (bound collection in BPI Joannes Kunze, Fungi Selecti Exsiccati 124, lectotype MBT178538, isolectotypes BPI 1108439, BPI 1108445), FRANCE, Veronnes, on vines of Hedera helix, 10 March 2011, A. Gardiennet (epitype BPI 892919, culture ex-epitype AR5211).

Host - Hedera helix (Udayanga et al. 2014b).

Distribution - France, Germany (Udayanga et al. 2014b).

69)Diaporthe hickoriae Wehm., Monogr. Gen. Diaporthe Nitschke \& Segreg. 9: 149 (1933), Mycobank MB252583.

Sexual morph not reported. See Wehmeyer (1933) for illustrations and descriptions of asexual morph.

Type - USA, Michigan, on Carya glabra (Juglandaceae), June 1926, L.E. Wehmeyer (holotype, culture ex-type CBS 145.26).

Host - Carya glabra (Wehmeyer 1933).

Distribution - USA (Wehmeyer 1933).

70)Diaporthe hongkongensis R.R. Gomes, C. Glienke \& Crous, Persoonia 31: 23 (2013), MycoBank MB802934.

Sexual morph not reported. See Gomes et al. (2013) for illustrations and descriptions of asexual morph.

Type - Hong Kong, Tai Po Kau, on fruit of Dichroa febrifuga (Hydrangeaceae), 20 Feb. 2002, K.D. Hyde (holotype CBS H-21103, culture ex-type CBS 115448).

Hosts - Camelia sinensis (Gao et al. 2016), Citrus sp. (Huang et al. 2015), Dichroa febrifuga (Gomes et al. 2013), Vitis vinifera (Dissanayake et al. 2015).

Distribution - China (Dissanayake et al. 2015, Huang et al. 2015, Gao et al. 2016), Hong Kong (Gomes et al. 2013).

71) Diaporthe incompleta Y.H. Gao \& L. Cai, IMA fungus 8: 175 (2017), MycoBank MB820681. Sexual morph not reported. See Gao et al. (2017) for illustrations and descriptions of asexual morph.

Type - China, Yunnan Province, Xishuangbanna, on diseased of Elaeagnus glabra (Elaeagnaceae), 19 Apr. 2015, F. Liu (holotype HMAS 247088, culture ex-type CGMCC 3.18288 $=$ LC 6754).

Hosts - Elaeagnus glabra (Gao et al. 2017). 
Distribution - China (Gao et al. 2017).

72)Diaporthe inconspicua R.R. Gomes, Glienke \& Crous, Persoonia 31: 23 (2013), MycoBank MB802936.

Cultures are sterile. See Gomes et al. (2013) for culture characteristics.

Type - Brazil, on petiole of Maytenus ilicifolia (Celastraceae), July 2007, R.R. Gomes (holotype CBS H-21102, culture ex-type CBS 133813).

Hosts - Maytenus ilicifolia, Spondias mombin (Gomes et al. 2013).

Distribution - Brazil (Gomes et al. 2013).

73)Diaporthe infecunda R.R. Gomes, C. Glienke \& Crous, Persoonia 31: 24 (2013), MycoBank MB802937.

Cultures are sterile. See Gomes et al. (2013) for culture characteristics.

Type - Brazil, on leaf of Schinus terebinthifolius (Anacardiaceae), July 2007, J. Lima (holotype CBS H-21095, culture ex-type CBS 133812).

Hosts - Maytenus ilicifolia, Phaseolus vulgaris, Schinus terebinthifolius (Gomes et al. 2013).

Distribution - Brazil (Gomes et al. 2013, dos Santos et al. 2016).

74) Diaporthe isoberliniae Crous, Persoonia 32: 221 (2014), MycoBank MB808909.

Sexual morph not reported. See Crous et al. (2014a) for illustrations and descriptions of asexual morph.

Type - Zambia, B\&B13467, on Isoberlinia angolensis (Fabaceae) 23 Feb. 2013, M. van der Bank (holotype CBS H-21693, culture ex-type CBS 137981).

Host - Isoberlinia angolensis (Crous et al. 2014a).

Distribution - Zambia (Crous et al. 2014a).

75) Diaporthe juglandicola C.M. Tian \& Q. Yang, Mycosphere 8: 821 (2017), Facesoffunginumber FoF03111, Index Fungorum number IF552939.

Sexual morph and asexual morph have been reported. See Yang et al. (2017) for illustrations and descriptions.

Type - China, Beijing City, Yanqing County, Songshan Nature Reserve, on twigs and branches of Juglans mandshurica, 9 May 2015, S.S. Hao (holotype BJFC-S1342, culture ex-type CFCC 51134).

Host - Juglans mandshurica (Yang et al. 2017).

Distribution - China (Yang et al. 2017).

76) Diaporthe kochmanii R.G. Shivas, S.M. Thomps. \& A.J. Young, Persoonia 27: 86 (2011), MycoBank MB561571.

Sexual morph and asexual morph have been reported. See Thompson et al. (2011) for illustrations and descriptions.

Type - Australia, Queensland, Lawes, on Helianthus annuus (Asteraceae) Experimental Line, 25 Nov. 2010, S.M. Thompson (holotype BRIP 54033, includes ex-type culture).

Host - Helianthus annuus (Thompson et al. 2011).

Distribution - Australia (Thompson et al. 2011).

77) Diaporthe kongii R.G. Shivas, S.M. Thomps. \& A.J. Young, Persoonia 27: 86 (2011), MycoBank MB561570.

Sexual morph not reported. See Thompson et al. (2011) for illustrations and descriptions of asexual morph.

Type - Australia, Queensland, Childers, on Helianthus annuus (Asteraceae) hybrid PDAS, 1 Dec. 2010, S.M. Thompson (holotype BRIP 54031, includes ex-type culture), Childers, on Helianthus annuus hybrid PDAS, 1 Dec. 2010, S.M. Thompson, paratype BRIP 54032. 
Host - Helianthus annuus (Thompson et al. 2011).

Distribution - Australia (Thompson et al. 2011).

78)Diaporthe litchicola R.G. Shivas, Grice \& Y.P. Tan, Fungal Diversity 61: 256 (2013), MycoBank MB802385.

Sexual morph not reported. See Tan et al. (2013) for illustrations and descriptions of asexual morph.

Type - Australia, Queensland, Mareeba, on Litchi chinensis (Sapindaceae), 22 Nov 2011, K.R.E. Grice (holotype BRIP 54900, includes ex-type culture).

Host - Litchi chinensis (Tan et al. 2013).

Distribution - Australia (Tan et al. 2013).

79) Diaporthe lithocarpus (Y.H. Gao, W. Sun \& L. Cai) Y.H. Gao \& L. Cai, Mycol Progress 13: 115 (2014), MycoBank MB802137.

Sexual morph not reported. See Gao et al. (2014) for illustrations and descriptions of asexual morph.

Type - China, Zhejiang Province, Gutianshan Nature Reserve, on leaves of Lithocarpus glabra (Fagaceae), 15 Aug. 2010, W Sun, LC0784 (holotype HMAS 244234, culture ex-type CGMCC 3.15175).

Host - Lithocarpus glabra (Gao et al. 2014).

Distribution - China (Gao et al. 2014).

80)Diaporthe longicicola Y.H. Gao \& L. Cai, Fungal Biology 119: 306 (2015), MycoBank MB805930.

Sexual morph not reported. See Gao et al. (2015) for illustrations and descriptions of asexual morph.

Type - China, Zhejiang Province, Gutianshan Nature Reserve, on leaves of Lithocarpus glabra (Fagaceae), 15 Aug. 2010, W. Sun (holotype HMAS 244839, culture ex-type CGMCC 3.17089).

Host - Lithocarpus glabra (Gao et al. 2015).

Distribution - Type (Gao et al. 2015).

81) Diaporthe longicolla (Hobbs) J.M. Santos, Vrandečić \& A.J.L. Phillips, Persoonia 27: 13 (2011), Mycobank MB563213.

Sexual morph not reported. See Santos et al. (2011) and Udayanga et al. (2015) for illustrations and descriptions of asexual morph.

Type - USA, Ohio, Wayne Co., Wooster, Ohio Agricultural Research and Development Center, on seeds of Glycine max cv. Wells (Fabaceae), 5 day old dried culture on PDA, 13 Nov. 1983, T.W. Hobbs P 74 (holotype BPI 358745, culture ex-type ATCC 60325).

Hosts - Recent studies subsequent to Udayanga et al. (2015) confirm the following hosts: Glycine max (Hernandez et al. 2015, Divilov et al. 2016), Pyrus sp. (Bai et al. 2015).

Distribution - Recent studies subsequent to Udayanga et al. (2015) confirm the following distribution: Argentina (Hernandez et al. 2015), China (Bai et al. 2015), USA (Divilov et al. 2016).

82) Diaporthe longispora (Wehm.) R.R. Gomes, C. Glienke \& Crous, Persoonia 31: 24 (2013), MycoBank MB802938.

See Gomes et al. (2013) for descriptions.

Type - Canada, Ontario, Toronto, on Ribes sp. (Grossulariaceae), May 1936, L.E. Wehmeyer (holotype CBS 194.36, includes ex-type culture).

Host - Ribes sp. (Gomes et al. 2013).

Distribution - Canada (Gomes et al. 2013). 
83)Diaporthe lonicerae Dissanayake, Camporesi \& K.D. Hyde, Mycosphere 8: 867 (2017), Facesoffunginumber FoF03273, Index Fungorum number IF553189.

Sexual morph not reported. See Dissanayake et al. (2017b) for illustrations and descriptions of asexual morph.

Type - Italy, Forlì-Cesena Province, Predappio Alta, on dead aerial branch of Lonicera sp. (Caprifoliaceae), 28 Febrary 2015, Erio Camporesi (holotype MFLU 15-3511, culture ex-type MFLUCC 17-0963).

Host - Lonicera sp. (Dissanayake et al. 2017b).

Distribution - Italy (Dissanayake et al. 2017b).

84)Diaporthe lusitanicae A.J.L. Phillips \& J.M. Santos, Fungal Diversity 34: 118 (2009), MycoBank MB512256.

Sexual morph and asexual morph have been reported. See Santos \& Phillips (2009) for illustrations and descriptions.

Type - Portugal, Lisbon, Oeiras, Estação Agronómica Nacional, stem of Foeniculum vulgare (Apiaceae), 14 Aug. 2007, J.M. Santos (holotype CBS 123212, includes ex-type culture).

Host - Foeniculum vulgare (Santos \& Phillips 2009).

Distribution - Portugal (Santos \& Phillips 2009).

85) Diaporthe macintoshii R.G. Shivas, S.M. Thomps. \& Y.P. Tan, Persoonia 35: 43 (2015), MycoBank MB808670.

Sexual morph not reported. See Thompson et al. (2015) for illustrations and descriptions of asexual morph.

Type - Australia, Queensland, Toowoomba, from stem of Rapistrum rugosum (Brassicaceae), 6 Dec. 2011, S.M. Thompson T12768A (holotype BRIP 55064a, includes ex-type culture).

Host - Rapistrum rugosum (Thompson et al. 2015).

Distribution - Australia (Thompson et al. 2015).

86) Diaporthe mahothocarpus Y.H. Gao, W. Sun \& L. Cai) Y.H. Gao \& L. Cai, Mycol Progress 13: 117 (2014), Mycobank MB802138.

Sexual morph not reported. See Gao et al. (2015) for illustrations and descriptions of asexual morph.

Type - China, Zhejiang Province, Gutianshan Nature Reserve, on leaves of Lithocarpus glabra (Fagaceae), 15 Aug. 2010, W Sun, LC0763, (holotype HMAS 244235, culture ex-type CGMCC 3.15181).

Host - Lithocarpus glabra (Gao et al. 2015).

Distribution - China (Gao et al. 2015).

87) Diaporthe malorum L. Santos \& A. Alves, Mycosphere 8: 494 (2017), MycoBank MB820226. Sexual morph not reported. See Santos et al. (2017) for illustrations and descriptions of asexual morph.

Type - Portugal, Felgueiras, from Malus domestica (Rosaceae) fruit with rot symptoms, January 2014, A. Alves, (holotype LISE 96314, culture extype CBS142383 = CAA734).

Host - Malus domestica (Santos et al. 2017).

Distribution - Portugal (Santos et al. 2017).

88) Diaporthe maritima J.B. Tanney, Fungal Biology 120: 1453 (2016), MycoBank MB816941.

Sexual morph not reported. See Tanney et al. (2016) for illustrations and descriptions of asexual morph.

Type - Canada, New Brunswick, Alma, Fundy National Park, Dickson's Falls, 45.58690 N, $64.97468 \mathrm{~W}, 100 \mathrm{~m}$ alt, isolated as endophyte from healthy surface-sterilized Picea rubens 
(Pinaceae) needle, 23 Sep. 2013, J.B. Tanney NB-365-71I (holotype DAOM695742, culture extype DAOMC 250563).

Host - Picea rubens (Tanney et al. 2016).

Distribution - Canada (Tanney et al. 2016).

89) Diaporthe masirevicii R.G. Shivas, L. Morin, S.M. Thompson \& Y.P. Tan, Persoonia 35: 45 (2015), MycoBank MB808671.

Sexual morph not reported. See Thompson et al. (2015) for illustrations and descriptions of asexual morph.

Type - Australia, Queensland, Glenore Grove, from the stem of Helianthus annuus (Asteraceae), 15 Aug. 2012, S.M. Thompson T13228C (holotype BRIP 57892a, includes ex-type culture).

Hosts - Chrysanthemoides monilifera, Glycine max, Helianthus annuus, Zea mays (Thompson et al. 2015).

Distribution - Australia (Thompson et al. 2015).

90)Diaporthe mayteni R.R. Gomes, Glienke \& Crous, Persoonia 31: 24 (2013), MycoBank MB802939.

Sexual morph not reported. See Gomes et al. (2013) for illustrations and descriptions of asexual morph.

Type - Brazil, Paraná, Colombo, endophytic species isolated from petiole of Maytenus ilicifolia (popular name Espinheira Santa, Celastraceae), July 2007, R.R. Gomes (holotype CBS H-21096, culture ex-type CBS 133185).

Hosts - Carapa guianensis (Ferreira et al. 2015), Maytenus ilicifolia (Gomes et al. 2013).

Distribution - Brazil (Gomes et al. 2013, Ferreira et al. 2015).

91) Diaporthe maytenicola Crous, Persoonia 31: 259 (2013), MycoBank MB805858.

Sexual morph not reported. See Crous et al. (2013) for illustrations and descriptions of asexual morph.

Type - South Africa, Western Cape Province, Kirstenbosch Botanical Garden, on leaves of Maytenus acuminata var. acuminata (Celastraceae), 29 Dec. 2012, P.W. Crous (holotype CBS H21452, culture ex-type CBS 136441).

Host - Maytenus acuminata var. acuminata (Crous et al. 2013).

Distribution - South Africa (Crous et al. 2013).

92)Diaporthe melonis Beraha \& M.J. O'Brien, Phytopath. Z. 94: 205 (1979), MycoBank MB312933.

Sexual morph and asexual morph have been reported. See Beraha \& O'Brien (1979) for illustrations and descriptions.

Type - USA, Texas, Rio Grande Valley, on Cucumis melo, 1978, L. Beraha \& M.J. O’Brien (isotype CBS H-891, culture ex-isotype CBS 507.78).

Hosts - Annona squamosa (Ola et al. 2014), Carapa guianensis (Ferreira et al. 2015), Cucumis melo (Beraha \& O'Brien 1979).

Distribution - Brazil (Ferreira et al. 2015), Germany (Ola et al. 2014), USA (Beraha \& O'Brien 1979).

93)Diaporthe middletonii R.G. Shivas, L. Morin, S.M. Thomps. \& Y.P. Tan, Persoonia 35: 45 (2015), MycoBank MB808672.

Sexual morph not reported. See Thompson et al. (2015) for illustrations and descriptions of asexual morph.

Type - Australia, Queensland, Gatton, from stem of Rapistrum rugosum (Brassicaceae), 24 Nov. 2011, S.M. Thompson T12757H (holotype BRIP 54884e, includes ex-type culture).

Hosts - Chrysanthemoides monilifera, Rapistrum rugostrum (Thompson et al. 2015). 
Distribution - Australia (Thompson et al. 2015).

94)Diaporthe miriciae R.G. Shivas, S.M. Thompson \& Y.P. Tan, Persoonia 35: 46 (2015), MycoBank MB808673.

Sexual morph not reported. See Thompson et al. (2015) for illustrations and descriptions of asexual morph.

Type - Australia, New South Wales, Premer, from stubble of Helianthus annuus (Asteraceae), 11 Aug. 2011, S.M. Thompson T12711M (holotype BRIP 54736j, includes ex-type culture).

Hosts - Glycine max, Helianthus annuus, Vigna radiate (Thompson et al. 2015).

Distribution - Australia (Thompson et al. 2015).

95)Diaporthe momicola Dissanayake, X.H. Li \& K.D. Hyde, Mycosphere 8: 541 (2017), Facesoffunginumber FoF01958, Index Fungorum number IF551987.

Sexual morph not reported. See Dissanayake et al. (2017a) for illustrations and descriptions of asexual morph.

Type - China, Hubei Province, on diseased shoots of Prunus persica (Rosaceae), May 2015, XingHong Li (holotype MFLU 16-0905, culture ex-type MFLUCC 16-0113=CGMCC 3.17466).

Host - Prunus persica (Dissanayake et al. 2017a).

Distribution - China (Dissanayake et al. 2017a).

96) Diaporthe multigutullata F. Huang, K.D. Hyde \& H.Y. Li, Fungal Biology 119: 343 (2015), MycoBank MB810581.

Sexual morph not reported. See Huang et al. (2015) for illustrations and descriptions of asexual morph.

Type - China, Fujian Province, Zhangzhou, on asymptomatic branch of Citrus grandis (Rutaceae), June 2011, F. Huang (holotye ZJUD98H, culture ex-type CGMCC 3.17258 = ICMP 20656).

Host - Citrus grandis (Huang et al. 2015).

Distribution - China (Huang et al. 2015).

97) Diaporthe musigena Crous \& R.G. Shivas, Persoonia 26: 119 (2011), MycoBank MB560160.

Sexual morph not reported. See Crous et al. (2011) for illustrations and descriptions of asexual morph.

Type - Australia, Queensland, Brisbane, on leaves of Musa sp. (Musaceae), 14 July 2009, P.W. Crous \& R.G. Shivas (holotype CBS H-20579, culture ex-type CBS 129519).

Host - Musa sp. (Crous et al. 2011).

Distribution - Australia (Crous et al. 2011).

98) Diaporthe neilliae Peck, Rep. (Annual) Trustees State Mus. Nat. Hist., New York 39: 52 (1887), Mycobank MB160628.

Sexual morph reported, asexual morph is not reported. See Udayanga et al. (2014b) for illustrations and descriptions of sexual morph.

Type - USA, New York, West Albany, on stems of Physocarpus opulifolius (Rosaceae), C.H. Peck USA, on Spiraea sp., September 1927, L.E. Wehmeyer (isotype BPI 616581, culture ex-isotype CBS 144.27).

Host - Physocarpus opulifolius (Udayanga et al. 2014b).

Distribution - USA (Udayanga et al. 2014b).

99)Diaporthe neoarctii R.R. Gomes, C. Glienke \& Crous, Persoonia 31: 26 (2013), MycoBank MB802940.

Sexual morph not reported. See Gomes et al. (2013) for illustrations and descriptions of asexual morph. 
Type - USA, New Jersey, isolated from Ambrosia trifida (Asteraceae), May 2001, G. Bills (holotype CBS H-21094, culture ex-type CBS 109490 = GB 6421 = AR 3450).

Host - Ambrosia trifida (Gomes et al. 2013).

Distribution - USA (Gomes et al. 2013).

100) Diaporthe neoraonikayaporum Doilom, Dissan. \& K.D. Hyde, Fungal Diversity 82: 161 (2016), Facesoffunginumber FoF01870, Index Fungorum number IF551993.

Sexual morph not reported. See Doilom et al. (2016) for illustrations and descriptions of asexual morph.

Type - Thailand, Chiang Rai Province, Mae Suai District, Mae Lao garden, on dieback lesion of Tectona grandis (Lamiaceae) branches, 5 July 2014, (holotype MFLU 15-3539, culture ex-type MFLUCC 14-1136, MKT 168/ 1, ICMP 21176, Chiang Rai Province, Muang District, Doi Lan Subdistrict, on dieback lesion of T. grandis twigs, 14 June 2014, M. Doilom, (paratype MFLU 153538, culture ex-paratype MFLUCC 14-1133).

Host - Tectona grandis (Doilom et al. 2016).

Distribution - Thailand (Doilom et al. 2016).

101) Diaporthe nobilis Sacc. \& Speg., Michelia 1: 386 (1878), Facesoffungi number FoF02717, Index Fungorum number 153616.

Sexual morph not reported. See Li et al. (2017) for illustrations and descriptions of asexual morph. Authentic strain - Japan, isolate from Pinus pentaphylla bonsai plant imported from Japan into the Netherlands, May 1979, G.H. Boerema (CBS H-16732, culture CBS 587.79).

Host - See Gomes et al. (2013) and Li et al. (2017).

Distribution - See Gomes et al. (2013) and Li et al. (2017).

102) Diaporthe nothofagi R.G. Shivas, J. Edwards \& Y.P. Tan, Fungal Diversity 61: 257 (2013), MycoBank MB802386.

Sexual morph not reported. See Tan et al. (2013) for illustrations and descriptions of asexual morph.

Type - Australia, Victoria, Carlton, on Nothofagus cunninghamii (Nothofagaceae), 31 Oct. 2000, C. Brenchley (holotype VPRI 22429b, includes ex-type culture), isotype BRIP 54801.

Host - Nothofagus cunninghamii (Tan et al. 2013).

Distribution - Australia (Tan et al. 2013).

103) Diaporthe novem J.M. Santos, Vrand. \& A.J.L. Phillips, Persoonia 27: 14 (2011), MycoBank MB518521.

Sexual morph and asexual morph have been reported. See Santos et al. (2011) for illustrations and descriptions.

Type - Croatia, Slavonija, on Glycine max seed (Fabaceae), Sept. 2008, T. Duvnjak (holotype CBS H-20463, culture ex-type CBS 127270).

Hosts - Actinidia sp. (Diaz et al. 2014), Asclepias syriaca, Aspalathus linearis (van Rensburg et al. 2006), Glycine max, Helianthus annuus (Rekab et al. 2004), Hydrangea macrophylla (Santos et al. 2011), Prunus dulcis (Lawrence et al. 2015), Vitis vinifera (van Niekerk et al. 2005).

Distribution - Chile (Diaz et al. 2014), Croatia, Italy (Rekab et al. 2004), Portugal (Santos et al. 2011), South Africa (van Niekerk et al. 2005, van Rensburg et al. 2006), USA (Lawrence et al. 2015).

104) Diaporthe ocoteae Crous \& M.J. Wingf., Persoonia 36: 397 (2016), MycoBank MB817058. Sexual morph not reported. See Crous et al. (2016) for illustrations and descriptions of asexual morph.

Type - France, La Réunion, on leaves of Ocotea obtusata (Lauraceae), 6 Mar. 2015, P.W. Crous \& M.J. Wingfield (holotype CBS H-22627, culture ex-type CBS 141330). 
Host v Ocotea obtusata (Crous et al. 2016).

Distribution - France (Crous et al. 2016).

105) Diaporthe oraccinii Y.H. Gao \& L. Cai, Systematics and Biodiversity 14: 111 (2016), MycoBank MB811233.

Sexual morph not reported. See Gao et al. (2016) for illustrations and descriptions of asexual morph.

Type - China, Jiangxi Province, Chongyi County, on healthy leaves of Camellia sinensis (Theaceae), 24 Apr. 2013, F. Liu (holotype HMAS 246031, culture ex-type CGMCC 3.17531).

Host - Camellia sinensis (Gao et al. 2016).

Distribution - China (Gao et al. 2016).

106) Diaporthe ovalispora F. Huang, K.D. Hyde \& H.Y. Li, Fungal Biology 119: 343 (2015), MycoBank MB810582.

Sexual morph not reported. See Huang et al. (2015) for illustrations and descriptions of asexual morph.

Type - China, Yunnan Province, Ruili, on nonsymptom twig of Citrus limon (Rutaceae), July 2011, F. Huang (holotype ZJUD93H, culture ex-type CGMCC 3.17256).

Host - Citrus limon (Huang et al. 2015).

Distribution - China (Huang et al. 2015).

107) Diaporthe ovoicicola Y.H. Gao \& L. Cai, Fungal Biology 119: 302 (2015), MycoBank MB805926.

Sexual morph not reported. See Gao et al. (2015) for illustrations and descriptions of asexual morph.

Type - China, Zhejiang Province, Gutianshan Nature Reserve, N29'20', E118'14', on leaves of Lithocarpus glabra (Fagaceae), 15 Aug. 2010, W. Sun (holotype HMAS 244835, culture ex-type CGMCC 3.17092).

Host - Lithocarpus glabra (Gao et al. 2015).

Distribution - China (Gao et al. 2015).

108) Diaporthe oxe R.R. Gomes, C. Glienke \& Crous, Persoonia 31: 28 (2013), MycoBank MB802941.

Sexual morph not reported. See Gomes et al. (2013) for illustrations and descriptions of asexual morph.

Type - Brazil, on petiole of Maytenus ilicifolia (Celastraceae), July 2007, R.R. Gomes (holotype CBS H-21098, culture ex-type CBS 133186).

Hosts - Maytenus ilicifolia, Schinus terebinthifolius (Gomes et al. 2013).

Distribution - Brazil (Gomes et al. 2013).

109) Diaporthe paranensis R.R. Gomes, Glienke \& Crous, Persoonia 31: 29 (2013), MycoBank MB802942.

Sexual morph not reported. See Gomes et al. (2013) for illustrations and descriptions of asexual morph.

Type - Brazil, Paraná, Colombo, endophytic species isolated from petiole of Maytenus ilicifolia (popular name Espinheira Santa, Celastraceae), July 2007, R.R. Gomes (holotype CBS H-21099, culture ex-type CBS 133184).

Host - Maytenus ilicifolia (Gomes et al. 2013).

Distribution - Brazil (Gomes et al. 2013).

110) Diaporthe parapterocarpi Crous, Persoonia 32: 229 (2014), MycoBank MB808917. 
Sexual morph not reported. See Crous et al. (2014a) for illustrations and descriptions of asexual morph.

Type - Zambia, OM4178, on Pterocarpus brenanii (Fabaceae), 1 Mar 2013, M. van der Bank (holotype CBS H-21698, culture ex-type CBS 137986).

Host - Pterocarpus brenanii (Crous et al. 2014a).

Distribution - Zambia (Crous et al. 2014a).

111) Diaporthe pascoei R.G. Shivas, Jacq. Edwards \& Y.P. Tan, Fungal Diversity 61: 258 (2013), MycoBank MB802387.

Sexual morph not reported. See Tan et al. (2013) for illustrations and descriptions of asexual morph.

Type - Australia, Victoria, on Persea Americana (Lauraceae), 29 Nov. 1988, I.G. Pascoe, (holotype VPRI 16058, includes ex-type cultures), isotype BRIP 54847.

Host - Persea americana (Tan et al. 2013).

Distribution - Australia (Tan et al. 2013).

112) Diaporthe passiflorae Crous \& L. Lombard Persoonia 28: 149 (2012), MycoBank MB800372.

Sexual morph not reported. See Crous et al. (2012) for illustrations and descriptions of asexual morph.

Type - South America, imported into the Netherlands, on fruit of Passiflora edulis (Passifloraceae), Apr. 2011, P.W. Crous (holotype CBS H-20956, culture ex-type CBS 132527).

Host - Passiflora edulis (Crous et al. 2012).

Distribution - South America (Crous et al. 2012).

113) Diaporthe passifloricola Crous \& M.J. Wingf., Persoonia 36: 395 (2016), MycoBank MB817057.

Sexual morph not reported. See Crous et al. (2016) for illustrations and descriptions of asexual morph.

Type - Malaysia, Kota Kinabalu, on leaf spots of Passiflora foetida (Passifloraceae), May 2015, M.J. Wingfield (holotype CBS H-22626, culture ex-type CBS 141329).

Host - Passiflora foetida (Crous et al. 2016).

Distribution - Malaysia (Crous et al. 2016).

114) Diaporthe penetriteum Y.H. Gao \& L. Cai, Systematics and Biodiversity 14: 112 (2016), MycoBank MB811218.

Sexual morph not reported. See Gao et al. (2016) for illustrations and descriptions of asexual morph.

Type - China, Jiangxi Province, Chongyi County, on diseased leaves of Camellia sinensis (Theaceae), 24 Apr. 2013, F. Liu, (holotype HMAS 245776, culture ex-type CGMCC 3.17532).

Host - Camellia sinensis (Gao et al. 2016).

Distribution - China (Gao et al. 2016).

115) Diaporthe perjuncta Niessl, Hedwigia 15: 153 (1876), Mycobank MB165386.

Sexual morph reported. See van Niekerk et al. (2005) for illustrations and descriptions of sexual morph.

Type - Austria, from Ulmus glabra (Ulmaceae), Oct. 2001, A.Y. Rossman (culture ex-epitype CBS 109745).

Host - Ulmus glabra (van Niekerk et al. 2005).

Distribution - Austria, Germany (van Niekerk et al. 2005). 
116) Diaporthe perseae (Zerova) R.R. Gomes, C. Glienke \& Crous, Persoonia 31: 29 (2013), MycoBank MB802944.

Sexual morph not reported. See Gomes et al. (2013) for illustrations and descriptions of asexual morph.

Type - Netherlands Antilles, Martinique, on young fruit of Persea gratissima (Lauraceae), 10 July 1972, E. Laville (holotype CBS 151.73, includes ex-type culture).

Host - Persea gratissima (Gomes et al. 2013).

Distribution - Netherlands (Gomes et al. 2013).

117) Diaporthe pescicola Dissanayake, X.H. Li \& K.D. Hyde, Mycosphere 8: 542 (2017), Facesoffunginumber FoF01959, Index Fungorum number IF551988.

Sexual morph not reported. See Dissanayake et al. (2017a) for illustrations and descriptions of asexual morph.

Type - China, Hubei Province, on diseased shoots of Prunus persica (Rosaceae), May 2015, XingHong Li (holotype MFLU 16-0906, culture ex-type MFLUCC 16-0105=CGMCC3.17465).

Host - Prunus persica (Dissanayake et al. 2017a).

Distribution - China (Dissanayake et al. 2017a).

118) Diaporthe phaseolorum (Cooke \& Ellis) Sacc., Syll. fung. (Abellini) 1: 692 (1882), Mycobank MB164797.

Sexual morph and asexual morph have been reported. See Santos et al. (2011) and Udayanga et al. (2015) for illustrations and descriptions.

Type - USA, on old vines of Phaseolus sp. (Fabaceae), 1878, Cooke and Ellis (In Ellis North American Fungi, Century II, No.188 in Exsiccati bound collection in BPI - lectotype; MBT200095), (BPI 616862-paratype), Delaware: Kent Co., Townsend, on bean pod and leaf of Phaseolus sp., 14 Jul. 2005, N. F. Gregory (epitype BPI 879942, ex-epitype).

Hosts - Ocimum sanctum (Chowdhary \& Kaushik 2015), Phaseolus sp. (Harter 1917, Udayanga et al. 2015, dos Santos et al. 2016), Vitis vinifera (Dissanayake et al. 2015).

Distribution - Brazil (dos Santos et al. 2016), China (Dissanayake et al. 2015), Cuba, Jamaica, USA (Delaware, New Jersey, Ohio) (Harter 1917, Udayanga et al. 2015), India (Chowdhary \& Kaushik 2015).

119) Diaporthe phragmitis Crous, Persoonia 33: 219 (2014), MycoBank MB810588.

Sexual morph not reported. See Crous et al. (2014b) for illustrations and descriptions of asexual morph.

Type - China, Beijing, Fragrant Hill, on Phragmites australis (Poaceae), 31 Aug. 2013, P.W. Crous \& Y. Zhang (holotype CBS H-21979, culture ex-type CPC 23607 = CBS 138897).

Host - Phragmites australis (Crous et al. 2014b).

Distribution - China (Crous et al. 2014b).

120) Diaporthe podocarpi-macrophylli Y.H. Gao \& L. Cai, IMA fungus 8: 176 (2017), MycoBank MB820682.

Sexual morph not reported. See Gao et al. (2017) for illustrations and descriptions of asexual morph.

Type - Japan, on healthy leaves of Podocarpus macrophyllus (Podocarpaceae), 20 Sep. 2014, W.J.

Duan (holotype HMAS 247084, culture ex-type CGMCC 3.18281 = LC 6155).

Host - Podocarpus macrophyllus (Gao et al. 2017).

Distribution - Japan (Gao et al. 2017).

121) Diaporthe pseudomangiferae R.R. Gomes, Glienke \& Crous, Persoonia 31: 30 (2013), MycoBank MB802945. 
Sexual morph not reported. See Gomes et al. (2013) for illustrations and descriptions of asexual morph.

Type - Dominican Republic, from Mangifera indica (Anacardiaceae), P. de Leeuw, ATO-DLO, Wageningen (holotype CBS H-21105, culture ex-type CBS 101339).

Host - Mangifera indica (Gomes et al. 2013, Serrato-Diaz et al. 2014).

Distribution - Dominican Republic (Gomes et al. 2013), Mexico (Gomes et al. 2013), USA (Serrato-Diaz et al. 2014).

122) Diaporthe pseudophoenicicola R.R. Gomes, Glienke \& Crous, Persoonia 31: 30 (2013), MycoBank MB803839.

Sexual morph not reported. See Gomes et al. (2013) for illustrations and descriptions of asexual morph.

Type - Spain, Mallorca, Can Pastilla, dead tops of green leaves on Phoenix dactylifera (Arecaceae), 27 May 1969, H.A. van der Aa (holotype CBS H-21106, culture ex-type CBS 462.69).

Hosts - Mangifera indica, Phoenix dactylifera (Gomes et al. 2013).

Distribution - Iraq, Spain (Gomes et al. 2013).

123) Diaporthe pseudotsugae Dissanayake, Camporesi \& K.D. Hyde, Mycosphere 8: 869 (2017), Facesoffunginumber FoF03274, Index Fungorum number IF553190.

Sexual morph not reported. See Dissanayake et al. (2017b) for illustrations and descriptions of asexual morph.

Type - Italy, Forlì-Cesena Province, Premilcuore, on dead land cones of Pseudotsuga menziesii (Pinaceae), 10 April 2015, Erio Camporesi (MFLU 15-1274, holotype).

Host - Pseudotsuga menziesii (Dissanayake et al. 2017b).

Distribution - Italy (Dissanayake et al. 2017b).

124) Diaporthe psoraleae Crous \& M.J. Wingf., Persoonia 31: 205 (2013), MycoBank MB805822.

Sexual morph not reported. See Crous et al. (2013) for illustrations and descriptions of asexual morph.

Type - South Africa, Western Cape Province, Betty's Bay, Harold Porter National Botanical Garden, on stems of Psoralea pinnata (Fabaceae), 28 Oct. 2012, M.J. Wingfield (holotype CBS H21422, culture ex-type CBS 136412).

Host - Psoralea pinnata (Crous et al. 2013).

Distribution - South Africa (Crous et al. 2013).

125) Diaporthe psoraleae-pinnatae Crous \& M.J. Wingf., Persoonia 31: 205 (2013), MycoBank MB805823.

Sexual morph not reported. See Crous et al. (2013) for illustrations and descriptions of asexual morph.

Type - South Africa, Western Cape Province, Betty's Bay, Harold Porter National Botanical Garden, on stems of Psoralea pinnata (Fabaceae), 28 Oct. 2012, M.J. Wingfield (holotype CBS H21423, culture ex-type CBS 136413).

Host - Psoralea pinnata (Crous et al. 2013).

Distribution - South Africa (Crous et al. 2013).

126) Diaporthe pterocarpi (S. Hughes) Udayanga, X.Z. Liu \& K.D. Hyde, Cryptog. Mycol. 33: 305 (2012), MycoBank MB801055.

Sexual morph not reported. See Udayanga et al. (2012b) for illustrations and descriptions of asexual morph. 
Type - Togoland (Togo, West Africa), Kete Krachi, on leaves of Pterocarpus erinaceus (Fabaceae), 18 April 1949, S Hughes (PDD 14878, isotype), Thailand, Chiang Rai Province, Mae Fah Luang University Garden, leaves of Pterocarpus indicus, 12 April 2010, D. Udayanga, DPH 002 (epitype MFLU 12-0120, culture ex-epitype MFLUCC 10-0571).

Hosts - Aloe vera, Jatropha curcas, Ougeinia dalbergioides, Pterocarpus santalinoides, P. angolensis, $P$. erinaceus, $P$. Indicus, $P$. violaceus (Udayanga et al. 2012b).

Distribution - Brazil, Ghana, Hong Kong, India, Sierra Leone, Thailand, Togo, Zambia (Udayanga et al. 2012b).

127) Diaporthe pterocarpicola Udayanga, X.Z. Liu \& K.D. Hyde, Cryptog. Mycol. 33: 303 (2012), MycoBank MB801053.

Sexual morph not reported. See Udayanga et al. (2012b) for illustrations and descriptions of asexual morph.

Type - Thailand, Chiang Rai Province, Thasud, Muang District, Chiang Rai Arboretum, N $18^{\circ} 05^{\prime}$ 59.1", E 102 ${ }^{\circ} 40^{\prime}$ 02.9", on leaves of Pterocarpus indicus (Fabaceae), 14 May 2010, D. Udayanga DPH 013 (holotype MFLU 12-0129, culture ex-type MFLUCC 10-0580a).

Host - Pterocarpus indicus (Udayanga et al. 2012b).

Distribution - Thailand (Udayanga et al. 2012b).

128) Diaporthe pulla Nitschke, Pyrenomyc. Germ. 2: 246 (1870), Mycobank MB168654.

Sexual morph not reported. See Udayanga et al. (2014b) for illustrations and descriptions of asexual morph.

Type - Germany, on vines of Hedera helix (Araliaceae), (Fries Scleromyceti Sueciae No. 307 (BPI Sbarbaro Collection, Bound, Centuries III (part) to V. in BPI as Sphaeria spiculosa, lectotypeMBT178540), Serbia, Belgrade, on vines of Hedera helix, July 1989, M. Muntañola-Cvetkovic (epitype BPI 892920, culture ex-epitype CBS 338.89).

Host - Hedera helix (Udayanga et al. 2014b).

Distribution - Czech Republic, Germany (Udayanga et al. 2014b).

129) Diaporthe pyracanthae L. Santos \& A. Alves, Mycosphere 8: 493 (2017), MycoBank MB820224.

Sexual morph not reported. See Santos et al. (2017) for illustrations and descriptions of asexual morph.

Type - Portugal, Aveiro, from branch canker of Pyracantha coccinea (Rosaceae), March 2012, A. Alves, (holotype LISE 96313, culture ex-type CBS142384 = CAA483).

Host - Pyracantha coccinea (Santos et al. 2017).

Distribution - Portugal (Santos et al. 2017).

130) Diaporthe raonikayaporum R.R. Gomes, C. Glienke \& Crous, Persoonia 31: 31 (2013), MycoBank MB802947.

Sexual morph not reported. See Gomes et al. (2013) for illustrations and descriptions of asexual morph.

Type - Brazil, Pará, Redenção, endophytic species isolated from leaf of Spondias mombin (popular name Cajazeira and Taperebá, Anacardiaceae), July 2007, K. Rodriguez (holotype CBS H-21097, culture ex-type CBS 133182).

Host - Spondias mombin (Gomes et al. 2013).

Distribution - Brazil (Gomes et al. 2013).

131) Diaporthe ravennica Thambugala, Camporesi \& K.D. Hyde, Fungal Diversity 82: 296 (2017), Facesoffunginumber FoF02171, Index Fungorum number IF552100.

Sexual morph not reported. See Thambugala et al. (2016) for illustrations and descriptions of asexual morph. 
Type - Italy, Province of Ravenna, Lido di Dante, on dead branches of Tamarix sp. (Tamaricaceae), 4 December 2014, Erio Camporesi IT 2273 (holotype MFLU 16-0665, culture extype MFLUCC 15-0479).

Host - Tamarix sp. (Thambugala et al. 2016).

Distribution - Italy (Thambugala et al. 2016).

132) Diaporthe rhusicola Crous, Persoonia 26: 135 (2011), MycoBank MB560170.

Sexual morph not reported. See Crous et al. (2011) for illustrations and descriptions of asexual morph.

Type - South Africa, Western Cape Province, Cape Town, Kirstenbosch Botanical Garden, on leaves of Rhus pendulina (White Karee, Anacardiaceae), 8 May 2010, P.W. Crous (holotype CBS H-20589, culture ex-type CBS 129528).

Hosts - Juglans regia (Chen et al. 2014), Rhus pendulina (Crous et al. 2011).

Distribution - California (Chen et al. 2014), South Africa (Crous et al. 2011).

133) Diaporthe rostrata C.M. Tian, X.L. Fan \& K.D. Hyde, Mycol. Progr. 14: 4 (2015), Mycobank MB812974.

Sexual morph and asexual morph have been reported. See Fan et al. (2015b) for illustrations and descriptions.

Type - China, Gansu Province, Tianshui City, Xiaolong Mountain, 3403'19.64" N, 10545'13.95"

E, $1432 \mathrm{~m}$ asl., on stems and branches of Juglans mandshurica (Juglandaceae), 19 July 2013, collected by X.L. Fan \& X.Y.Wu (holotype BJFC-S890, culture ex-type CFCC 50062).

Host - Juglans mandshurica (Fan et al. 2015b).

Distribution - China (Fan et al. 2015b).

134) Diaporthe rudis (Fr.) Nitschke, Pyrenomyc. Germ. 2: 282 (1870), Mycobank MB139900.

Sexual morph and asexual morph have been reported. See Udayanga et al. (2014a) for illustrations and descriptions.

Type - Austria, Vienna, 19. 7763/2, Reisenbergbach-Weg, on stem of Laburnum anagyroides (Fabaceae), 8 Apr. 2000, W. Jaklitsch (epitype BPI 748231, culture ex-epitype AR3422).

Hosts - See Udayanga et al. (2014a).

Distribution - See Udayanga et al. (2014a).

135) Diaporthe saccarata (J.C. Kang, L. Mostert \& Crous) Crous, Persoonia 31: 32 (2013), MycoBank MB802948.

Sexual morph not reported. See Gomes et al. (2013) for illustrations and descriptions of asexual morph.

Type - South Africa, Western Cape Province, Jonkershoek Mountains, Stellenbosch, on cankers of Protea repens (Proteaceae), Mar. 1999, S. Denman (culture ex-type CBS).

Host - Protea repens (Gomes et al. 2013).

Distribution - South Africa (Gomes et al. 2013).

136) Diaporthe sackstonii R.G. Shivas, S.M. Thompson \& Y.P. Tan, Persoonia 35: 46 (2015), MycoBank MB808674.

Sexual morph not reported. See Thompson et al. (2015) for illustrations and descriptions of asexual morph.

Type - Australia, Queensland, Clermont, from a petiole of Helianthus annuus (Asteraceae), 10 June 2011, S.M. Thompson T12667B (holotype BRIP 54669b, includes ex-type culture).

Host - Helianthus annuus (Thompson et al. 2015).

Distribution - Australia (Thompson et al. 2015). 
137) Diaporthe salicicola R.G. Shivas, J. Edwards \& Y.P. Tan, Fungal Diversity 61: 258 (2013), MycoBank MB803338.

Sexual morph not reported. See Tan et al. (2013) for illustrations and descriptions of asexual morph.

Type - Australia, Tasmania, Blackfish Creek, on Salix purpurea (Salicaceae), 31 July 2007, K. Finlay \& R. Adair (holotype VPRI 32789, includes ex-type culture), isotype BRIP 54825.

Host - Salix purpurea (Tan et al. 2013).

Distribution - Australia (Tan et al. 2013).

138) Diaporthe schini R.R. Gomes, C. Glienke \& Crous, Persoonia 31: 32 (2013), MycoBank MB802949.

Sexual morph not reported. See Gomes et al. (2013) for illustrations and descriptions of asexual morph.

Type - Brazil, Paraná, Curitiba, endophytic species isolated from leaf of Schinus terebinthifolius (popular name Aroeira, Anacardiaceae), July 2007, J. Lima (holotype CBS H-21093, culture extype CBS 133181).

Host - Schinus terebinthifolius (Gomes et al. 2013).

Distribution - Brazil (Gomes et al. 2013).

139) Diaporthe schoeni Dissanayake, Camporesi \& K.D. Hyde, Mycosphere 8: 870 (2017), Facesoffunginumber FoF03275, Index Fungorum number IF553191.

Sexual morph not reported. See Dissanayake et al. (2017b) for illustrations and descriptions of asexual morph.

Type - Italy, Ravenna Province, Lido di Dante, on dead aerial stem of Schoenus nigricans (Cyperaceae), 1 May 2015, Erio Camporesi (holotype MFLU 15-1279).

Host - Schoenus nigricans (Dissanayake et al. 2017b).

Distribution - Italy (Dissanayake et al. 2017b).

140) Diaporthe sclerotioides (Kesteren) Udayanga, Crous \& K.D. Hyde, Fungal Diversity 56: 166 (2012), Mycobank MB800700.

Sexual morph not reported. See van Kesteren (1967) for illustrations and descriptions of asexual morph.

Type - Netherlands, Maarssen, on root of Cucumis sativus (Cucurbitaceae), June 1967, H.A. van der Kesteren (culture ex-type CBS 296.67).

Hosts - Cucumis sativus (van Kesteren 1967, Shishido et al. 2014), Citrullus lanatus, C. ficifolia, C. maxima, C. moschata (Udayanga et al. 2011, Shishido et al. 2014), Cucumis melo, Citrullus lanatus, Lagenaria siceraria var. gourda (Shishido et al. 2014).

Distribution - Netherlands (van Kesteren 1967), Japan (Shishido et al. 2014).

141) Diaporthe sennae C.M. Tian \& Q. Yang, Phytotaxa 302: 149 (2017), MycoBank MB820452. Sexual morph not reported. See van Yang et al. (2017) for illustrations and descriptions of asexual morph.

Type - China, Guangxi Province, Nanning city, on twigs and branches of Senna bicapsularis, Q. Yang, 7 November 2015 (holotype BJFC-S1370, culture ex-type CFCC51636).

Host - Senna bicapsularis (Yang et al. 2017).

Distribution - China (Yang et al. 2017).

142) Diaporthe sennicola C.M. Tian \& Q. Yang, Phytotaxa 302: 150 (2017), MycoBank MB820453.

Sexual morph not reported. See van Yang et al. (2017) for illustrations and descriptions of asexual morph. 
Type - China, Guangxi Province, Nanning city, on twigs and branches of Senna bicapsularis, Q. Yang, 7 November 2015 (holotype BJFC-S1369, culture ex-type CFCC51635).

Host - Senna bicapsularis (Yang et al. 2017).

Distribution - China (Yang et al. 2017).

143) Diaporthe serafiniae R.G. Shivas, S.M. Thompson \& Y.P. Tan, Persoonia 35: 46 (2015), MycoBank MB808675.

Sexual morph not reported. See Thompson et al. (2015) for illustrations and descriptions of asexual morph.

Type - Australia, Queensland, Glenore Grove, from seed of an ornamental variety of Helianthus annuus (Asteraceae), 1 Apr. 2012, S.M. Thompson T13010A (holotype BRIP 55665b, includes extype culture).

Hosts - Helianthus annuus, Lupinus albus, Lupinus Serafin (Thompson et al. 2015).

Distribution - Australia (Thompson et al. 2015).

144) Diaporthe siamensis Udayanga, X.Z. Liu \& K.D. Hyde, Cryptog. Mycol. 33: 298 (2012), MycoBank MB 800826.

Sexual morph not reported. See Udayanga et al. (2012b) for illustrations and descriptions of asexual morph.

Type - Thailand, Chiang Rai Province, Thasud, Muang District, Mae Fah Luang University Park, N $18^{\circ} 05^{\prime}$ 59.1", E 102 40'02.9”, on leaves of Dasymaschalon sp. (Annonaceae), 11 March 2010, D. Udayanga DPH 004 (holotype MFLU 12-0121, culture ex-type MFLUCC 10-0573a).

Host - Dasymaschalon sp. (Udayanga et al. 2012b).

Distribution - Thailand (Udayanga et al. 2012b).

145) Diaporthe sojae Lehman, Ann. Mo. bot. Gdn 10: 128 (1923), Mycobank MB278338.

Sexual morph and asexual morph have been reported. See Udayanga et al. (2015) for illustrations and descriptions.

Type - USA, North Carolina, Raleigh, on stems and pods of Glycine max (Fabaceae), 1 Sep. 1920, S. G. Lehman. Perithecia developing on sterilized petioles of soybean after inoculation of pure culture of strain 17 with stems and pods (lectotype BPI 615412, MBT 200097), on stems and pods of Glycine max, Late summer, 1920, S.G. Lehman, (isolectotype BPI 615415), Ohio, on Glycine max, unknown dates and collection data (epitype BPI 748002, culture ex-epitype FAU635).

Hosts - Camptotheca acuminata (Chang et al. 2005), Capsicum annuum (Pennycook 1989), Citrus sp. (Huang et al. 2015), Cucumis melo, Glycine max (Lehman 1923, Zhang et al. 1997, Santos et al. 2011), Helianthus annuus (Vrandecic et al. 2004, Thompson et al. 2011), Stokesia laevis (Sogonov et al. 2008), Vitis vinifera (Dissanayake et al. 2015).

Distribution - Recent studies subsequent to Udayanga et al. (2015) confirm the following distribution: China (Dissanayake et al. 2015, Huang et al. 2015).

146) Diaporthe spartinicola Crous \& R.K. Schumach., Sydowia 67: 98 (2015), MycoBank MB812526.

Sexual morph not reported. See Crous et al. (2015b) for illustrations and descriptions of asexual morph.

Type - Spain, Jaén, Los Villares, on stem of Spartium junceum (Leguminosae), 24 June 2014, leg. S. Tello (holotype CBS H-22256, culture ex-type CBS 140003).

Host - Spartium junceum (Crous et al. 2015b).

Distribution - Spain (Crous et al. 2015b).

147) Diaporthe sterilis L. Lombard, Polizzi \& Crous, Phytopath. Mediterr. 53: 94 (2014), MycoBank MB807600. 
Sexual morph not reported. See Lombard et al. (2014) for illustrations and descriptions of asexual morph.

Type - Italy, Sicily, Catania Province, Valverde, on Vaccinium corymbosum (Ericaceae), June 2012, G. Polizzi (holotype CBS H-21515, culture ex-type CBS 136969).

Host - Vaccinium corymbosum (Lombard et al. 2014).

Distribution - Italy (Lombard et al. 2014).

148) Diaporthe stictica (Berk. \& Broome) R.R. Gomes, C. Glienke \& Crous, Persoonia 31: 34 (2013), MycoBank MB802950.

Culture sterile. See Gomes et al. (2013).

Type - Italy, Perugia, on dead twig of Buxus sempervirens (Buxaceae), Dec. 1954, M. Ribaldi (holotype CBS 370.54, includes ex-type culture).

Host - Buxus sempervirens (Gomes et al. 2013).

Distribution - Italy (Gomes et al. 2013).

149) Diaporthe subclavata F. Huang, K.D. Hyde \& H.Y. Li, Fungal Biology 119: 343 (2015), MycoBank MB810583.

Sexual morph not reported. See Huang et al. (2015) for illustrations and descriptions of asexual morph.

Type - China, Fujian Province, Yongchun, on leaf with citrus scab of Citrus unshiu (Rutaceae), May 2011, F. Huang and X. Hou (holotype ZJUD95H, culture ex-type ZJUD95).

Host - Citrus unshiu (Huang et al. 2015).

Distribution - China (Huang et al. 2015).

150) Diaporthe subordinaria (Desm.) R.R. Gomes, C. Glienke \& Crous, Persoonia 31: 34 (2013), MycoBank MB802951.

See notes in Gomes et al. (2013).

Type - New Zealand, blackened seed of Plantago lanceolata (Plantaginaceae), Apr. 1999, B. Alexander (holotype CBS 101711, includes extype culture).

Host - Plantago lanceolata (Gomes et al. 2013).

Distribution - New Zealand (Gomes et al. 2013).

151) Diaporthe taoicola Dissanayake, X.H. Li \& K.D. Hyde, Mycosphere 8: 543 (2017), Facesoffunginumber FoF01960, Index Fungorum number IF551989.

Sexual morph not reported. See Dissanayake et al. (2017a) for illustrations and descriptions of asexual morph.

Type - China, Hubei Province, on diseased shoots of Prunus persica (Rosaceae), July 2015, XingHong Li (holotype MFLU 16-0907, culture ex-type MFLUCC 16-0117=CGMCC3.17464).

Host - Prunus persica (Dissanayake et al. 2017a).

Distribution - China (Dissanayake et al. 2017a).

152) Diaporthe tectonae Doilom, Dissan. \& K.D. Hyde, Fungal Diversity 82: 164 (2016), Facesoffunginumber FoF01871, Index Fungorum number IF551976.

Sexual morph not reported. See Doilom et al. (2016) for illustrations and descriptions of asexual morph.

Type - Thailand, Phayao Province, Chun District, Hong Hin Subdistrict, on dieback lesion of Tectona grandis twig (Lamiaceae), 23 November 2012, M. Doilom \& J. Roux, (holotype MFLU 15-3535, culture ex-type MFLUCC 12-0777).

Host - Tectona grandis (Doilom et al. 2016).

Distribution - Thailand (Doilom et al. 2016). 
153) Diaporthe tectonendophytica Doilom, Dissan. \& K.D. Hyde, Fungal Diversity 82: 163 (2016), Facesoffunginumber FoF01869, Index Fungorum number IF551975.

Sexual morph not reported. See Doilom et al. (2016) for illustrations and descriptions of asexual morph.

Type - Thailand, Chiang Rai Province, Muang District, on asymptomatic branches of Tectona grandis (Lamiaceae), 19 March 2013, M. Doilom, (holotype MFLU 15-3537, culture ex-type MFLUCC 13-0471).

Host - Tectona grandis (Doilom et al. 2016).

Distribution - Thailand (Doilom et al. 2016).

154) Diaporthe tectonigena Doilom, Dissan. \& K.D. Hyde, Fungal Diversity 82: 165 (2016), Facesoffunginumber FoF01872, Index Fungorum number IF551977.

Sexual morph not reported. See Doilom et al. (2016) for illustrations and descriptions of asexual morph.

Type - Thailand, Chiang Rai Province, Muang District, on dieback lesions of Tectona grandis (Lamiaceae) twigs, 19 November 2012, M. Doilom, (holotype MFLU 15-3534, culture ex-type MFLUCC 12-0767).

Host - Tectona grandis (Doilom et al. 2016).

Distribution - Thailand (Doilom et al. 2016).

155) Diaporthe terebinthifolii R.R. Gomes, Glienke \& Crous, Persoonia 31: 35 (2013), MycoBank MB802952.

Sexual morph not reported. See Gomes et al. (2013) for illustrations and descriptions of asexual morph.

Type - Brazil, Paraná, Curitiba, endophytic species isolated from leaf of Schinus terebinthifolius (popular name Aroeira, Anacardiaceae), July 2007, J. Lima (holotype CBS H-21097, culture extype CBS 133180).

Host - Schinus terebinthifolius (Gomes et al. 2013).

Distribution - Brazil (Gomes et al. 2013).

156) Diaporthe ternstroemia Y.H. Gao, W. Sun \& L. Cai, Mycol. Progr. 13: 119 (2013), MycoBank MB802139.

Sexual morph not reported. See Gao et al. (2014) for illustrations and descriptions of asexual morph.

Type - China, Zhejiang Province, Gutianshan Nature Reserve, on leaves of Ternstroemia gymnanthera (Pentaphylacaceae), 15 Aug. 2010, W Sun (holotype HMAS 244234, LC0784, culture ex-type CGMCC 3.15183).

Host - Ternstroemia gymnanthera (Gao et al. 2014).

Distribution - China (Gao et al. 2014).

157) Diaporthe thunbergii Udayanga, X.Z. Liu \& K.D. Hyde, Cryptogamie Mycologie 33: 301 (2012), MycoBank MB800959.

Sexual morph not reported. See Udayanga et al. (2012b) for illustrations and descriptions of asexual morph.

Type - Thailand, Chiang Mai Province, Doi Suthep-Pui National Park, Medicinal Garden, $18^{\circ} 48.62 \mathrm{~N} 98^{\circ} 54.60 \mathrm{E}$, on leaves of Thunbergia laurifolia (Acanthaceae), 7 April 2010, D. Udayanga (holotype MFLU 12-0117, culture ex-type MFLUCC 10-0576a).

Host - Thunbergia laurifolia (Udayanga et al. 2012b).

Distribution - Thailand (Udayanga et al. 2012b).

158) Diaporthe thunbergiicola Udayanga \& K.D. Hyde, in Liu et al., Fungal Diversity 72: 22 (2015), Facesoffunginumber FoF00472, Index Fungorum number IF551072. 
Sexual morph not reported. See Liu et al. (2015) for illustrations and descriptions of asexual morph. Type - Thailand, Chiang Mai Province, Doi Suthep Pui herbal garden, on leaves of Thunbergia laurifolia (Acanthaceae), 10 January 2012, D. Udayanga DPH 114 (holotype MFLU 14-0816, culture ex-type MFLUCC 12-0033).

Host - Thunbergia laurifolia (Liu et al. 2015).

Distribution - Thailand (Liu et al. 2015).

159) Diaporthe torilicola Dissanayake, Camporesi \& K.D. Hyde, Mycosphere 8: 870 (2017), Facesoffunginumber FoF03276, Index Fungorum number IF553192.

Sexual morph not reported. See Dissanayake et al. (2017b) for illustrations and descriptions of asexual morph.

Type - Italy, Forlì-Cesena Province, Monte Pallareto - Meldola dead aerial stem of Torilis arvensis (Apiaceae), 12 April 2016, Erio Camporesi (holotype MFLU 16-1166, culture ex-type MFLUCC 17-1051).

Host - Torilis arvensis (Dissanayake et al. 2017b).

Distribution - Italy (Dissanayake et al. 2017b).

160) Diaporthe toxica P.M. Will., Highet, W. Gams \& Sivasith., Mycol. Res. 98: 1367 (1994), MycoBank MB363488

See Williamson et al. (1994) for illustration and description of sexual morph. Asexual morph not reported.

Type - Western Australia, Morawa, on stem of Lupinus angustifolius (Fabaceae), 6 May 1991, J.B. Nunn (culture ex-type CBS 534.93 = ATCC 96741), Serpentine, on Lupinus sp., 8 June 1993, P.M. Williamson (CBS 535.93), Medina, on Lupinus sp., 8 June 1993, P.M. Williamson (CBS 546.93).

Host - Lupinus angustifolius (Ostazeski \& Wells 1960, Williamson et al. 1994, Gomes et al. 2013). Distribution - Australia, USA (Ostazeski \& Wells 1960, Williamson et al. 1994, Gomes et al. 2013).

161) Diaporthe tulliensis R.G. Shivas, Vawdrey \& Y.P. Tan, Persoonia 35: 301 (2015), MycoBank MB812896.

Sexual morph not reported. See Crous et al. (2015a) for illustrations and descriptions of asexual morph.

Type - Australia, Queensland, Tully, from rotted stem end of fruit of Theobroma cacao (Malvaceae), 10 Feb. 2015, M. Smith (holotype BRIP 62248a, includes ex-type culture).

Host - Theobroma cacao (Crous et al. 2015a).

Distribution - Australia (Crous et al. 2015a).

162) Diaporthe ueckerae Udayanga \& Castl., Fungal Biology 119: 401 (2014), MycoBank MB810794.

Sexual morph not reported. See Udayanga et al. (2015) for illustrations and descriptions of asexual morph.

Type - USA, Oklahoma, on crown of Cucumis melo (Cucurbitaceae), F.A. Uecker (holotype BPI 748011, culture ex-type CBS 139283).

Hosts - Camellia sinensis (Gao et al. 2016), Cucumis melo (Udayanga et al. 2015).

Distribution - China (Gao et al. 2016), USA (Udayanga et al. 2015).

163) Diaporthe undulata Y.H. Gao \& L. Cai, IMA fungus 8: 178 (2017), MycoBank MB820683.

Sexual morph not reported. See Gao et al. (2017) for illustrations and descriptions of asexual morph.

Type - China-Laos border, on diseased leaves of unknown host, 19 Apr. 2014, F. Liu (holotype HMAS 247091, culture ex-type CGMCC 3.18293 = LC 6624).

Host - unknown host (Gao et al. 2017). 
Distribution - China-Laos border (Gao et al. 2017).

164) Diaporthe unshiuensis F. Huang, K.D. Hyde \& H.Y. Li, Fungal Biology 119: 344 (2015), MycoBank MB810845.

Sexual morph not reported. See Huang et al. (2015) for illustrations and descriptions of asexual morph.

Type - China, Zhejiang Province, Linhai, on melanose fruit of Citrus unshiu (Rutaceae), 2009, G.Q. Chen and F. Huang (holotype ZJUD52H, culture ex-type ZJUD52).

Host - Citrus unshiu (Huang et al. 2015).

Distribution - China (Huang et al. 2015).

165) Diaporthe vaccinii Shear, United States Department of Agriculture Technical Bulletin 258: 1 (1931), MycoBank MB281247.

Sexual morph not reported. See Farr et al. (2002) for illustrations and descriptions of asexual morph.

Type - USA, Massachusetts, on Oxycoccus macrocarpos (Ericaceae), Mar. 1932, C.L. Shear (culture ex-type CBS 160.32).

Host - Vaccinium sp. (Lombard et al. 2014, Udayanga et al. 2014b).

Distribution - Europe (Lombard et al. 2014), USA (Udayanga et al. 2014b).

166) Diaporthe vangueriae Crous, Persoonia 32: 227 (2014), MycoBank MB808916.

Sexual morph not reported. See Crous et al. (2014a) for illustrations and descriptions of asexual morph.

Type - Zambia, on twigs of Vangueria infausta (Rubiaceae) 20 Feb 2012, M. van der Bank (holotype CBS H-21697, culture ex-type CBS 137985).

Host - Vangueria infausta (Crous et al. 2014a).

Distribution - Zambia (Crous et al. 2014a).

167) Diaporthe vawdreyi Y.P. Tan \& R.G. Shivas, Persoonia 35: 303 (2015), MycoBank MB812895.

Sexual morph not reported. See Crous et al. (2015a) for illustrations and descriptions of asexual morph.

Type - Australia, Queensland, East Feluga, from fruit rot of Psidium guajava (Myrtaceae), 18 Sept. 2014, Y. Diczbalis (holotype BRIP 57887a, includes ex-type culture).

Host - Psidium guajava (Crous et al. 2015a).

Distribution - Australia (Crous et al. 2015a).

168) Diaporthe velutina Y.H. Gao \& L. Cai, IMA fungus 8: 178 (2017), MycoBank MB820684.

Sexual morph not reported. See Gao et al. (2017) for illustrations and descriptions of asexual morph.

Type - China, Jiangxi Province, on diseased leaves of Neolitsea sp. (Lauraceae), 5 Sep. 2013, Y.H. Gao (holotype HMAS 247087, culture ex-type CGMCC 3.18286 = LC 4421).

Host - Neolitsea sp. (Gao et al. 2017).

Distribution - China (Gao et al. 2017).

169) Diaporthe virgiliae N.M. Machingambi, L.L. Dreyer \& F. Roets, Pl. Path. 64: 1153 (2015), MycoBank MB811040.

Sexual morph not reported. See Machingambi et al. (2015) for illustrations and descriptions of asexual morph.

Type - South Africa, Western Cape Province, the Harold Porter National Botanical Garden, from roots of Virgilia oroboides (Fabaceae). April 2011, N. Machingambi (National Collection of 
Fungi, Pretoria, South Africa). (holotype PREM 61104, culture ex-type CBS 138788), April 2011, N. Machingambi, paratype PREM 61103, living cultures CMW $40748=$ CBS 138789.

Host - Virgilia oroboides (Machingambi et al. 2015).

Distribution - South Africa (Machingambi et al. 2015).

170) Diaporthe xishuangbanica Y.H. Gao \& L. Cai, IMA fungus 8: 179 (2017), MycoBank MB820685

Sexual morph not reported. See Gao et al. (2017) for illustrations and descriptions of asexual morph.

Type - China, Yunnan Province, Xishuangbanna, on diseased leaves of Camellia sinensis (Theaceae), 19 Apr. 2015, F. Liu (holotype HMAS 247083, culture ex-type CGMCC 3.18283 = LC 6744).

Host - Camellia sinensis (Gao et al. 2017).

Distribution - China (Gao et al. 2017).

171) Diaporthe yunnanensis Y.H. Gao \& L. Cai, IMA fungus 8: 180 (2017), MycoBank MB820686.

Sexual morph not reported. See Gao et al. (2017) for illustrations and descriptions of asexual morph.

Type - China, Yunnan Province, Xishuangbanna, on healthy leaves of Coffea sp., 20 Sep. 2014, W.J. Duan (holotype HMAS 247096, culture ex-type CGMCC 3.18289 = LC6168).

Host - Coffea sp. (Gao et al. 2017).

Distribution - China (Gao et al. 2017).

\section{Acknowledgements}

This work was financed by JNKYT201605, Innovation funds of IPEP, BAAFS and CARS-30. Alan J.L. Phillips acknowledges the support from Biosystems and Integrative Sciences Institute (BioISI, FCT/UID/ Multi/04046/2013).

\section{References}

Akay S, Ekiz G, Kocabas F, Kocabaş E et al. 2011 - Secondary metabolites from Phomopsis amygdali, an endophytic fungus isolated from hazelnut (Corylus avellana). Planta Medica 77, 1286-1287.

Akgul DS, Savas NG, Teker T, Keykubat B et al. 2015 - Fungal trunk pathogens of Sultana Seedless vineyards in Aegean region of Turkey. Phytopathologia Mediterranea 54, 380-393.

Annesi T, Luongo L, Vitale S, Galli M, Belisario A. 2016 - Characterization and pathogenicity of Phomopsis theicola anamorph of Diaporthe foeniculina causing stem and shoot cankers on sweet chestnut in Italy. Journal of Phytopathology 164, 412-416.

Arnold RH. 1975 - Diaporthe alleghaniensis. Fungi Canadenses 70, 1-2.

Auger J, Perez I, Esterio M. 2013 - Diaporthe ambigua associated with post-harvest fruit rot of kiwifruit in Chile. Plant Disease 97, 843-843.

Bai Q, Zhai L, Chen X, Hong N et al. 2015 - Biological and molecular characterization of five Phomopsis species associated with pear shoot canker in China. Plant Disease 99, 1704-1712.

Baumgartner K, Fujiyoshi PT, Travadon R, Castlebury LA et al. 2013 - Characterization of species of Diaporthe from wood cankers of grape in eastern North American vineyards. Plant Dis 97, 912-920.

Beraha LR, O’Brien MJ. 1979 - Diaporthe melonis sp. nov., a new soft rot of market cantaloupes. Phytopathologische Zeitschrift 94, 199-207. 
Bienapfl, Y. Balci. 2013 - Phomopsis blight, a new disease of Pieris japonica caused by Phomopsis amygdali in the United States. Plant Disease 97, 1403-1407.

Bradley CA, Li S. 2006 - First report of northern stem canker of soybean caused by Diaporthe phasealorum var. caulivora in North Dakota. Plant Disease 90, 687-687.

Castlebury LA, Farr DF, Rossman AY, Jaklitsch WJ. 2003 - Diaporthe angelicae comb. nov., a modern description and placement of Diaporthopsis in Diaporthe. Mycoscience 44, 203-208.

Chang CQ, Xi PG, Xiang MM, Jiang ZD, Chi PK. 2005 - New species of Phomopsis on woody plants in Hunan Province. Mycosystema 2, 145-154.

Chen SF, Morgan DP, Michailides TJ. 2014 - Botryosphaeriaceae and Diaporthaceae associated with panicle and shoot blight of pistachio in California, USA. Fungal Diversity 67, 157-179.

Chi P, Jiang Z, Xiang M. 2007 - Flora Fungorum Sinicorum 34: Phomopsis. Science, Beijing

Chiesa MA, Pioli RN, Cambursano MV, Morandi EN. 2013 - Differential expression of distinct soybean resistance genes interacting with Argentinean isolates of Diaporthe phaseolorum var. meridionalis. European Journal of Plant pathology 135, 351-362.

Chowdhary K, Kaushik N. 2015 - Fungal endophyte diversity and bioactivity in the Indian medicinal plant Ocimum sanctum Linn. Plos One 10, Article Number, e0141444.

Cinelli T, Mondello V, Marchi G, Burruano S et al. 2016 - First report of Diaporthe eres associated with cane blight of grapevine (Vitis vinifera) in Italy. Plant Disease 100, 532-532.

Costamilan LM, Yorinori JT, Almeida AMR, Seixas CDS et al. 2008 - First report of Diaporthe phaseolorum var. caulivora infecting soybean plants in Brazil. Tropical Plant Pathology 33, 381-385.

Crous PW, Phillips AJL, Baxter AP. 2000 - Phytopathogenic fungi from South Africa. University of Stellenbosch, Department of Plant Pathology Press.

Crous PW, Groenewald JZ, Shivas RG, Edwards J et al. 2011 - Fungal Planet description sheets, 69-91. Persoonia 26, 108-156.

Crous PW, Summerell BW, Shivas RG, Burgess TI et al. 2012 - Fungal Planet description sheets, 107-127. Persoonia 28, 138-182.

Crous PW, Wingfield MJ, Guarro J, Cheewangkoon R et al. 2013 - Fungal Planet description sheets, 154-213. Persoonia 31, 188-296.

Crous PW, Shivas RG, Quaedvlieg W, van der Bank M et al. 2014a - Fungal Planet description sheets, 214-280. Persoonia 32, 184-306.

Crous PW, Wingfield MJ, Schumacher RK, Summerell BA et al. 2014b - Fungal Planet description sheets, 281-319. Persoonia 33, 212-292.

Crous PW, Schumacher RK, Wingfield MJ, Lombard L et al. 2015a - Fungal Systematics and Evolution, FUSE 1. Sydowia 67, 81-118.

Crous PW, Wingfield MJ, Le Roux JJ, Richardson DM et al. 2015b - Fungal Planet description sheets, 371-399. Persoonia 35, 264-327.

Crous PW, Wingfield MJ, Richardson DM, Le Roux JJ et al. 2016 - Fungal Planet description sheets, 400-468. Persoonia 36, 316-458.

Cummings JA, Bergstrom GC. 2015 - First report of northern stem canker caused by Diaporthe caulivora in soybean in New York. Plant Disease 99, 1039-1040.

Dai FM, Zeng R, Lu JP. 2012 - First report of twig canker on peach caused by Phomopsis amygdali in China. Plant Disease 96, 288-289.

Dennis RWG. 1986 - Fungi of the Hebrides. Royal Botanic Gardens, Kew.

Diaz GA, Latorre BA, Jara S, Ferrada E et al. 2014 - First report of Diaporthe novem causing postharvest rot of kiwifruit during controlled atmosphere storage in Chile. Plant Disease 98, 1274-1275.

Diogo ELF, Santos JM, Phillips AJL. 2010 - Phylogeny, morphology and pathogenicity of Diaporthe and Phomopsis species on almond in Portugal. Fungal Diversity 44, 107-115.

Dissanayake AJ, Liu M, Zhang W, Chen Z et al. 2015 - Morphological and molecular characterization of Diaporthe species associated with grapevine trunk disease in China. Fungal Biology 119, 283-294. 
Dissanayake AJ, Zhang W, Liu M, Hyde KD et al. 2017a - Diaporthe species associated with peach tree dieback in Hubei, China. Mycosphere 8, 533-549.

Dissanayake AJ, Camporesi E, Hyde KD, Zhang Wei et al. 2017b - Molecular phylogenetic analysis reveals seven new Diaporthe species from Italy. Mycosphere 8, 853-877.

Divilov K, Walker DR. 2016 - Reaction of Diaporthe longicolla to a strain of Sarocladium kiliense. Biocontrol Science and Technology 26, 938-950.

Doilom M, Dissanayake AJ, Wanasinghe DN, Boonmee S et al. 2016 - Microfungi on Tectona grandis (teak) in Northern Thailand. Fungal Diversity 82, 107-182.

dos Santos TT, Leite S, de Souza LT, de Queiroz CB et al. 2016 - High genetic variability in endophytic fungi from the genus Diaporthe isolated from common bean (Phaseolus vulgaris L.) in Brazil. Journal of Applied Microbiology 120, 388-401.

Du Z, Fan XL, Hyde KD, Yang Q et al. 2016 - Phylogeny and morphology reveal two new species of Diaporthe from Betula spp. in China. Phytotaxa 269, 090-102.

Early MP, Punithalingam E. 1972 - Phomopsis anacardii sp. nov. on Anacardium occidentale. Transactions of British Mycological Society 59, 345-347.

Elfar K, Torres R, Diaz GA, Latorre BA. 2013 - Characterization of Diaporthe australafricana and Diaporthe spp. associated with stem canker of blueberry in Chile. Plant Disease 97, 10421050.

Evidente A, Rodeva R, Andolfi A. Stoyanova Z et al. 2011 - Phytotoxic polyketides produced by Phomopsis foeniculi, a strain isolated from diseased Bulgarian fennel. European journal of Plant Pathology. 130, 173-182.

Fan XL, Hyde KD, Udayanga D, Wu XY et al. 2016 - Diaporthe rostrata, a novel ascomycete from Juglans mandshurica associated with walnut dieback. Mycological Progress 14, 82.

Farr DF, Castlebury LA, Rossman AY. 2002 - Morphological and molecular characterization of Phomopsis vaccinii and additional isolates of Phomopsis from blueberry and cranberry in the eastern United States. Mycologia 94, 494-504.

Ferreira MC, Almeida V, de Lourdes Zani CL, de Almeida Alves TM et al. 2015 - Molecular phylogeny, diversity, symbiosis and discover of bioactive compounds of endophytic fungi associated with the medicinal Amazonian plant Carapa guianensis Aublet (Meliaceae). Biochemical Systematics and Ecology 59, 36-44.

Gao YH, Sun W, Su YY, Cai L. 2014 - Three new species of Phomopsis in Gutianshan Nature Reserve in China. Mycol Progress 13, 111-121.

Gao YH, Su YY, Sun W. 2015 - Diaporthe species occurring on Lithocarpus glabra in China, with descriptions of five new species. Fungal Biology 119, 295-309.

Gao YH, Liu F, Cai L. 2016 - Unravelling Diaporthe species associated with Camellia. Systematics and Biodiversity 14, 102-117.

Gao Y, Liu F, Duan W, Crous PW, Cai L. 2017 - Diaporthe is paraphyletic. IMA fungus 8, 153187.

Ginns JH. 1986 - Compendium of plant disease and decay fungi in Canada 1960-1980. Res Br Can Agric Publ 1813,416

Gomes RR, Glienke C, Videira SIR, Lombard L et al. 2013 - Diaporthe, a genus of endophytic, saprobic and plant pathogenic fungi. Persoonia 31, 1-41.

Gramaje D, Agusti-Brisach C, Perez-Sierra A, Moralejo E et al. 2012 - Fungal trunk pathogens associated with wood decay of almond trees on Mallorca (Spain). Persoonia 28, 1-13.

Grijalba P, Ridao A del C. 2012 - Survival of Diaporthe phaseolorum var. caulivora (causal agent of soybean stem canker) artificially inoculated in different crop residues. Tropical Plant Pathology 37, 271-274.

Grijalba P, Ridao A del C. 2014 - Growth rate and pathogenicity of isolates of Diaporthe phaseolorum var. caulivora. Phyton-International journal of experimental boatany 83, 325332.

Guerrero J, Perez S. 2013 - First report of Diaporthe australafricana caused stem canker and dieback in european hazelnut (Corylus avellana L.) in Chile. Plant disease 97, 1657-1657. 
Guillin EA, Grijalba PE, de Oliveira LO, Gottlieb AM. 2014 - Specific boundaries between the causal agents of the soybean stem canker. Tropical Plant Pathology 39, 316-325.

Hall TA. 1999 - BioEdit: a user-friendly biological sequence alignment editor and analysis program for Windows 95/98/NT. Nucleic Acids Symposium Series 41, 95-98.

Harter LL. 1917 - Pod and stem blight of Lima beans. Journal of Agricultural Research. Washington DC 11, 473-506.

Hernandez FE, Pioli RN, Peruzzo AM, Formento ÁN, Pratta GR. 2015 - Morphologic and molecular characterization of Phomopsis longicolla (teleomorph unknown, Diaporthales) from tempered and subtropical regions of Argentina. Revista De Biologia Tropical 63, 871884.

Hu DM, Cai L, Hyde KD. 2012 - Three new ascomycetes from freshwater in China. Mycologia 104, 1478-1489.

Huang F, Hou X, Dewdney MM, Fu Y et al. 2013 - Diaporthe species occurring on citrus in China. Fungal Diversity 61, 237-250.

Huang F, Udayanga D, Wang X, Hou X et al. 2015 - Endophytic Diaporthe associated with Citrus, A phylogenetic reassessment with seven new species from China. Fungal Biology 119, 331347.

Hyde KD, McKenzie EHC, KoKo TW. 2011 - Towards incorporating anamorphic fungi in a natural classification checklist and notes for 2010. Mycosphere 2, 1-88

Hyde KD, Nilsson RH, Alias SA, Ariyawansa HA et al. 2014 - One stop shop: backbones trees for important phytopathogenic genera: I. Fungal Diversity 67, 21-125.

Hyde KD, Hongsanan S, Jeewon R, Bhat DJ et al. 2016 - Fungal diversity notes 367-492, taxonomic and phylogenetic contributions to fungal taxa. Fungal Diversity 80, 1-270

Katoh K, Toh H. 2008 - Recent developments in the MAFFT multiple sequence alignment program. Briefings in Bioinformatics 9, 276-285.

Kowalski T, Kraj W, Bednarz B. 2016 - Fungi on stems and twigs in initial and advanced stages of dieback of European ash (Fraxinus excelsior) in Poland. European Journal of Forest research $135,565-579$.

Latorre BA, Elfar K, Espinoza JG, Torres R, Díaz GA. 2012 - First report of Diaporthe australafricana associated with stem canker on blueberry in Chile. Plant Disease 96, 768.

Lawrence DP, Travadon R, Baumgartner K. 2015 - Diversity of Diaporthe species associated with wood cankers of fruit and nut crops in northern California. Mycologia 107, 926-940.

Lehman SG. 1923 - Pod and stem blight of soybean. Annals of Missouri Botanical Gardens 10, 111-178.

Liu JK, Hyde KD, Jones EBG, Ariyawansa HA et al. 2015 - Fungal diversity notes 1-110, taxonomic and phylogenetic contributions to fungal species. Fungal Diversity 72, 1-197.

Li Y, Tan P, Zhao DG. 2017 - Diaporthe nobilis, a new record on Camellia sinensis in Guizhou Province, China. Mycosphere 8, 1-8.

Lombard L, van Leeuwen GCM, Guarnaccia V, Polizzi G et al. 2014 - Diaporthe species associated with Vaccinium, with specific reference to Europe. Phytopathologia mediterranea 53, 287-299.

Machingambi NM, Dreyer LL, Oberlander KC. Roux J, Roets F. 2015 - Death of endemic Virgilia oroboides trees in South Africa caused by Diaporthe virgiliae sp nov. Plant Pathology 64, 1149-1156.

Maharachchikumbura SSN, Hyde KD, Jones EBG, McKenzie EHC et al. 2015 - Towards a natural classification and backbone tree for Sordariomycetes. Fungal Diversity 72, 199-301.

Maharachchikumbura SSN, Hyde KD, Jones EBG, McKenzie EHC et al. 2016 - Families of Sordariomycetes. Fungal Diversity 79, 1-317.

Marincowitz S, Crous PW, Groenewald JZ, Wingfield MJ. 2008 - Microfungi occurring on the Proteaceae in the fynbos. CBS Biodiversity Series 7, 1-166. CBS Fungal Biodiversity Centre, Utrecht, Netherlands. 
Mathew FM, Alananbeh KM, Jordahl JG, Meyer SM et al. 2015b - Phomopsis stem canker, A reemerging threat to sunflower (Helianthus annuus) in the United States. Phytopathology 105, 990-997.

Mathew FM, Rashid KY, Gulya TJ, Markell SG. 2015a - First report of Phomopsis stem canker of sunflower (Helianthus annuus) caused by Diaporthe gulyae in Canada. Plant Disease 99, $160-160$.

Menard L, Brandeis PE, Simoneau P, Poupard P et al. 2014 - First report of umbel browning and stem necrosis caused by Diaporthe angelicae on carrot in France. Plant Disease 98, 421-422.

Mengistu A, Castlebury L, Smith R, Ray J, Bellaloui N. 2009 - Seasonal progress of Phomopsis longicolla infection on soybean plant parts and its relationship to seed quality. Plant Disease 93, 1009-1018.

Mostert L, Crous PW, Kang JC, Phillips AJL. 2001a - Species of Phomopsis and a Libertella sp. occurring on grapevines with specific reference to South Africa, morphological, cultural, molecular and pathological characterization. Mycologia 93, 146-167.

Mostert L, Kang JC, Crous PW, Denman S. 2001b - Phomopsis saccharata sp. nov., causing a canker and dieback disease of Protea repens in South Africa. Sydowia 53, 227-235.

Nitschke T. 1867 - Pyrenomycetes Germanici. Die Kernpilze Deutschlands, Breslau 1.

Nitschke T. 1870 - Pyrenomycetes Germanici 2, 161-320 Breslau. Eduard Trewendt, Germany

Nylander JAA. 2004 - MrModeltest 2.0. Program distributed by the author. Evolutionary Biology Centre, Uppsala University.

Ola ARB, Debbab AK, Tibor H, Brötz-Oesterhelt H et al. 2014 - Dihydroanthracenone metabolites from the endophytic fungus Diaporthe melonis isolated from Annona squamosa. Tetrahedron Letters 55, 3147-3150.

Ormeno-Nunez J, Reeleder RD, Watson AK. 1988 - A new species of Phomopsis recovered from field bindweed (Convolvulus arvensis). Canadian Journal of Botany 66, 2228-2233.

Ostazeski SA, Wells HD. 1960 - A phomopsis stem blight of yellow lupins (Lupinus luteus L.). Plant Disease Reporter 44, 66-67.

Pennycook SR. 1989 - Plant Diseases Recorded in New Zealand, vol. 3, Plant Disease Division, DSIR, Auckland, New Zealand.

Petrovic K, Vidic M, Riccioni L, Đorđević V, Rajković D. 2015 - First report of Diaporthe eres species complex causing seed decay of soybean in Serbia. Plant Disease 99, 1186-1186.

Phillips AJL. 2003 - Morphological characterization of Diaporthe foeniculacea and its Phomopsis anamorph on Foeniculum vulgare. Sydowia 55, 274-285.

Pioli R, Morandi EN, Bisaro V - 2001. First report of soybean stem canker caused by Diaporthe phaseolorum var. caulivora in Argentina. Plant Disease 85, 95.

Polonio JC, Almeida TT, Garcia A, Mariucci GEG et al. 2015 - Biotechnological prospecting of foliar endophytic fungi of guaco (Mikania glomerata Spreng.) with antibacterial and antagonistic activity against phytopathogens. Genetics and Molecular Research 14, 72977309.

Rehner SA, Uecker FA. 1994 - Nuclear ribosomal internal transcribed spacer phylogeny and host diversity in the coelomycete Phomopsis. Can J Bot 72, 1666-1674.

Rekab D, Sorbo G del, Reggio C, Zoina A, Firrao G. 2004 - Polymorphisms in nuclear rDNA and mtDNA reveal the polyphyletic nature of isolates of Phomopsis pathogenic to sunflower and a tight monophyletic clade of defined geographic origin. Mycological Research 108, 393-402.

Rodeva R, Gabler J. 2011 - Umbel Browning and Stem Necrosis, a New Disease of Fennel in Bulgaria. Journal of Phytopathology 159, 184-187.

Ronquist F, Huelsenbeck JP. 2003 - MrBayes 3: Bayesian phylogenetic inference under mixed models. Bioinformatics 19, 1572-1574.

Santos JM, Correia VG, Phillips AJL. 2010. Primers for mating-type diagnosis in Diaporthe and Phomopsis, their use in teleomorph induction in vitro and biological species definition. Fungal Biology 114, 255-270. 
Santos JM, Phillips AJL. 2009 - Resolving the complex of Diaporthe (Phomopsis) species occurring on Foeniculum vulgare in Portugal. Fungal Diversity 34, 111-125.

Santos JM, Vrandecic K, Cosic J, Duvnjak T, Phillips AJL. 2011 - Resolving the Diaporthe species occurring on soybean in Croatia. Persoonia 27, 9-19.

Santos L, Phillips AJL, Crous PW, Alves A. 2017 - Diaporthe species on Rosaceae with descriptions of D. pyracanthae sp. nov. and D. malorum sp. nov. Mycosphere 8, 485-511.

Sato T, Aoki M, Aoki T, Kubota M et al. 2014 - Fungi isolated from spoiled bean sprouts in Japan. Jarq-Japan Agricultural Research Quarterly 48, 317-329.

Serrato-Diaz LM, Rivera-Vargas LI, French-Monar RD. 2014 - First report of Diaporthe pseudomangiferae causing inflorescence rot, rachis canker, and flower abortion of mango. Plant Disease 98, 1004-1005.

Shaw CG. 1973 - Host fungus index for the Pacific Northwest - I. Hosts. Washington State University Agricultural Experiment Station Bulletin 765, 1-121.

Shishido M, Ohashi T, Momma N. 2014 - Diaporthe sclerotioides exhibits no host specificity among cucurbit species. Plant Pathology 63, 1357-1364.

Silvestro D, Michalak I. 2010 - RaxmlGUI: a graphical front-end for RAxML. Available from: http://sourceforge.net/projects/raxmlgui/ (accessed 15 June 2017).

Smit WA, Wingfield MJ, Wingfield BD. 1996 - A new canker disease of apple, pear, and plum rootstocks caused by Diaporthe ambigua in South Africa. Plant Disease 80, 1331-1335.

Sogonov MV, Castlebury LA, Rossman AY, Mejia LC, White JF. 2008 - Leaf inhabiting genera of the Gnomoniaceae, Diaporthales. Stud Mycol 62, 1-79.

Stravinskiene V, Snieskiene V, Stankeviciene A. 2015 - Health condition of Tilia cordata Mill. trees growing in the urban environment. Urban Forestry and Urban Greening 14, 115-122.

Sun S, Van K, Kim Moon Y, Min KH et al. 2012 - Diaporthe phaseolorum var. caulivora, a causal agent for both stem canker and seed decay on soybean. Plant Pathology Journal 28, 55-59.

Sutton BC. 1980 - The coelomycetes. Fungi imperfecti with pycnidia, acervuli and stromata. Commonwealth Mycological Institute, Kew, Surrey, England

Swofford DL. 2003 - PAUP*: phylogenetic analysis using parsimony and other methods, version 4 Sinauer Associates, Sunderland, Massachusetts.

Tai FL. 1979 - Sylloge Fungorum Sinicorum. Science Press, Academica Sinica, Peking.

Tan YP, Edwards J, Grice KRE, Shivas RG. 2013 - Molecular phylogenetic analysis reveals six new Diaporthe species from Australia. Fungal Divers 61, 251-260.

Tanney JB, McMullin DR, Green BD, Miller JD, Seifert KA. 2016 - Production of antifungal and antiinsectan metabolites by the Picea endophyte Diaporthe maritima sp. nov. Fungal Biology $120,1448-1457$.

Thambugala KM, Daranagama DA, Phillips AJL, Bulgakov TS et al. 2016 - Microfungi on Tamarix. Fungal Diversity 82, 239-306.

Thompson SM, Tan YP, Young AJ, Neate SM et al. 2011 - Stem cankers on sunflower (Helianthus annuus) in Australia reveal a complex of pathogenic Diaporthe (Phomopsis) species. Persoonia 27, 80-89.

Thompson SM, Tan YP, Shivas RG, Neate SM et al. 2015 - Green and brown bridges between weeds and crops reveal novel Diaporthe species in Australia. Persoonia 35, 39-49.

Udayanga D, Xingzhong L, McKenzie EHC, Chukeatirote E et al. 2011 - The genus Phomopsis, biology, applications, species concepts and names of common pathogens. Fungal Diversity $50,189-225$.

Udayanga D, Liu XZ, Crous PW, McKenzie EHC et al. 2012a - A multi-locus phylogenetic evaluation of Diaporthe (Phomopsis). Fungal Diversity 56, 157-171.

Udayanga D, Liu XZ, MCkenzie EHC, Chukeatirote E, Hyde KD. 2012b - Multi-locus phylogeny reveals three new species of Diaporthe from Thailand. Cryptogamie Mycologie 33, 295-309.

Udayanga D, Castlebury LA, Rossman AY, Hyde KD. 2014a - Species limits in Diaporthe, molecular re-assessment of D. citri, D. cytosporella, D. foeniculina and D. rudis. Persoonia $32,83-101$. 
Udayanga D, Castlebury LA, Rossman AY, Chukeatirote E, Hyde KD. 2014b - Insights into the genus Diaporthe, phylogenetic species delimitation in the D. eres species complex. Fungal Diversity 67, 203-229.

Udayanga D, Castlebury LA, Rossman AY, Chukeatirote E, Hyde KD. 2015 - The Diaporthe sojae species complex, phylogenetic re-assessment of pathogens associated with soybean, cucurbits and other field crops. Fungal Biology 119, 383-407.

Urbez-Torres JR, Peduto F, Smith RJ, Gubler WD. 2013 - Phomopsis dieback, a grapevine trunk disease caused by Phomopsis viticola in California. Plant Disease 97, 1571-1579.

Van Kesteren HA. 1967 - "Black root rot" in Cucurbitaceae caused by Phomopsis sclerotioides nov. spec. Netherlands Journal of Plant Pathology 73, 112-116.

van Niekerk JM, Groenewald JZ, Farr DF, Fourie PH et al. 2005 - Reassessment of Phomopsis species on grapevines. Australasian Plant Pathology 34, 27-39.

van Rensburg JCJ, Lamprecht SC, Groenewald JZ, Castlebury LA, Crous PW. 2006 Characterization of Phomopsis spp. associated with dieback of rooibos (Aspalathus linearis) in South Africa. Studies in Mycology 55, 65-74.

Vrandečić K, Cosic J, Riccioni L, Duvnjak T, Jurkovic D. 2004 - Phomopsis longicolla a new pathogen on Abutilon theophrasti in Croatia. Plant Pathology 53, 251.

Vrandečić K, Cosic J, Riccioni L, Duvnjak T, Jurkovic D. 2005 - Isolation of Diaporthe phaseolorum var. caulivora from Abutilon theophrasti in Croatia. Plant Pathology 54, 576576.

Weber GF. 1933 - Stem canker of Crotalaria spectabilis caused by Diaporthe crotalariae. Phytopathology 23, 602.

Wehmeyer LE. 1933 - The genus Diaporthe Nitschke and its segregates. University of Michigan Studies Scientific Series 9, 1-349.

Williamson PM, Higher AS, Gams W, Sivasithamparam K, Cowling WA. 1994 - Diaporthe toxica sp. nov. the cause of lupinosis in sheep. Mycology Research 98, 1364-1368.

Yang Q, Fan XL, Du Z, Tian CM. 2017 - Diaporthe juglandicola sp. nov. (Diaporthales, Ascomycetes) evidenced by morphological characters and phylogenetic analysis. Mycosphere $8,817-826$.

Zhang AW, Hartman GL, Riccioni L, Chen WD et al. 1997 - Using PCR to distinguish Diaporthe phaseolorum and Phomopsis longicolla from other soybean fungal pathogens and to detect them in soybean tissues. Plant Disease 81, 1143-1149.

Zhu L, Li HY. 2010 - First report of Phomopsis amygdali causing shoot blight of peach in China. Journal of Plant Pathology 92, 112-112. 